\title{
REL LEAVES OF THE ARNOUX-YOCCOZ SURFACES
}

\author{
W. PATRICK HOOPER AND BARAK WEISS, WITH AN APPENDIX BY \\ LIOR BARY-SOROKER, MARK SHUSTERMAN, AND UMBERTO ZANNIER
}

\begin{abstract}
We analyze the rel leaves of the Arnoux-Yoccoz translation surfaces. We show that for any genus $\mathbf{g} \geqslant 3$, the leaf is dense in the connected component of the stratum $\mathcal{H}(\mathbf{g}-1, \mathbf{g}-1)$ to which it belongs, and the one-sided imaginary-rel trajectory of the surface is divergent. For one surface on this trajectory, namely the ArnouxYoccoz surface itself, the horizontal foliation is invariant under a pseudo-Anosov map (and in particular is uniquely ergodic), but for all other surfaces, the horizontal foliation is completely periodic. The appendix proves a field theoretic result needed for denseness of the leaf: for any $n \geqslant 3$, the field extension of $\mathbb{Q}$ obtained by adjoining a root of $X^{n}-X^{n-1}-\ldots-X-1$ has no totally real subfields other than $\mathbb{Q}$.
\end{abstract}

\section{INTRODUCTION}

A translation surface is a compact oriented surface equipped with a geometric structure which is Euclidean everywhere except at finitely many cone singularities and which has trivial rotational holonomy around loops. A stratum is a moduli space of translation surfaces of the same genus whose singularities share the same combinatorial characteristics. In recent years, intensive study has been devoted to the study of dynamics of group actions and foliations on strata of translation surfaces. See $\$ 2$ for precise definitions, and see [MaTa, $\mathrm{Z}$ ] for surveys.

Let $x$ be a translation surface with $k>1$ singularities. There is a local deformation of $x$ obtained by moving its singularities with respect to each other while keeping the translational holonomies of closed curves on $x$ fixed. This local deformation gives rise to a foliation of the stratum $\mathcal{H}$ containing $x$, with leaves of real dimension $2(k-1)$. In the literature this foliation has appeared under various names (see [Zo, §9.6], Sch], [McM4] and references therein), and we refer to it as the rel foliation, since nearby surfaces in the same leaf differ only in their relative periods. A sub-foliation of this foliation, which we will refer

Date: September 5, 2021. 


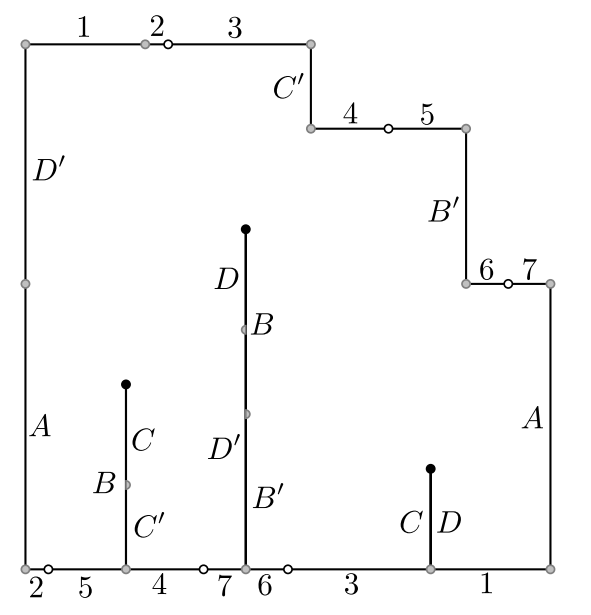

\begin{tabular}{ll} 
Label & Edge length \\
\hline 1 & $\frac{1-\alpha}{2}$ \\
2 & $\alpha-\frac{1}{2}$ \\
3 & $\frac{\alpha}{2}$ \\
4,5 & $\frac{\alpha^{2}}{2}$ \\
6,7 & $\frac{\alpha^{3}}{2}$ \\
$A$ & $\alpha$ \\
$B$ & $\frac{\alpha+\alpha^{3}}{2}$ \\
$B^{\prime}$ & $\alpha^{2}$ \\
$C, D$ & $\frac{\alpha^{2}+\alpha^{4}}{2}$ \\
$C^{\prime}$ & $\alpha^{3}$ \\
$D^{\prime}$ & $\alpha^{2}+\alpha^{3}$
\end{tabular}

Figure 1. The Arnoux-Yoccoz surface of genus $\mathbf{g}=$ 3 with distinguished singularities and the presentation from [Arn2, pp. 496-498]. Edges with the same label are identified by translation, and their lengths are provided by the chart. The points $\bullet$ and $\circ$ are the two singularities of the surface. Grey dots denote regular points.

to as the real-rel foliation, is obtained by only varying the horizontal holonomies of vectors, keeping all vertical holonomies fixed. The imaginary-rel foliation is defined by switching the roles of horizontal and vertical. Although none of these foliations are given by a group action, the obstructions to flowing along the real-rel and imaginaryrel foliations are completely understood (see [MW]). Specializing to $k=2$, it makes sense to discuss the real-rel trajectory $\left\{\operatorname{Rel}_{r}^{(h)} x: r \in \mathbb{R}\right\}$ (respectively, the imaginary-rel trajectory $\left\{\operatorname{Rel}_{s}^{(v)} x: s \in \mathbb{R}\right\}$ ) of any surface $x$ without horizontal (resp. vertical) saddle connections joining distinct labeled singularities.

A primary purpose of this paper is to contribute to the study of the topology of closures of rel leaves. We focus on the rel leaf of the Arnoux-Yoccoz surface $x_{0}$ of genus $\mathbf{g} \geqslant 3$ (see Figure 1 for a picture when $\mathbf{g}=3$, and see $\$ 4$ for the definition), which lies in the stratum $\mathcal{H}(\mathbf{g}-1, \mathbf{g}-1)$. Note that the surface depends on $\mathbf{g}$ but we suppress $\mathrm{g}$ from the notation. We also discuss a related rel leaf in the genus 2 stratum $\mathcal{H}(1,1)$. These surfaces, introduced by Arnoux and Yoccoz in [AY], are a source of interesting examples for the theory of translation surfaces as we discuss in 1.1 .3 . 
Our main results are stated below. Refer to \$2 for detailed definitions, to $\$ 1.1$ for connections to other mathematical ideas, and to $\$ 1.2$ for an outline of the proofs.

Theorem 1.1. Suppose $\mathbf{g} \geqslant 3$ and $x_{0}$ is the Arnoux-Yoccoz surface in genus $\mathbf{g}$. Then the imaginary-rel trajectory of $x_{0}$ is divergent in $\mathcal{H}$. Moreover, for any $s>0$, there is a horizontal cylinder decomposition of $\operatorname{Rel}_{s}^{(v)} x_{0}$, and the circumferences of the horizontal cylinders tend uniformly to zero as $s \rightarrow+\infty$.

The pseudo-Anosov map of [AY] preserves both the horizontal and vertical directions on $x_{0}$. Nevertheless, the vertical and horizontal directions play a different role in Theorem 1.1. In case $\mathbf{g}=3$ the surface $x_{0}$ has additional symmetries (see [Bow2]), and exploiting them we obtain the following stronger statement:

Corollary 1.2. Suppose $\mathbf{g}=3$. Then for any $s \neq 0$, the surface $\operatorname{Rel}_{s}^{(v)} x_{0}$ has a horizontal cylinder decomposition and the surface $\operatorname{Rel}_{s}^{(h)} x_{0}$ has a vertical cylinder decomposition. As $s \rightarrow+\infty$ and as $s \rightarrow-\infty$, the circumferences of the cylinders in these decompositions tend uniformly to zero.

Remark 1.3. In genus $\mathbf{g}=4$, experimental evidence (attained with the sage-flatsurf software package $[\mathrm{DH}]$ ) suggests that $\operatorname{Rel}_{s}^{(v)} x_{0}$ is divergent in $\mathcal{H}$ in negative time as well, though not all $\operatorname{Rel}_{s}^{(v)} x_{0}$ with $s<0$ admit horizontal cylinder decompositions. It would be interesting to understand the behavior of $\operatorname{Rel}_{s}^{(v)} x_{0}$ as $s \rightarrow-\infty$ and the behavior of $\operatorname{Rel}_{s}^{(h)} x_{0}$ as $s \rightarrow \pm \infty$. The present article sheds little light on this.

The behavior of the full rel leaf is very different. We will denote by $\mathcal{H}_{0}$ the subset of the connected component of $\mathcal{H}(\mathbf{g}-1, \mathbf{g}-1)$ containing $x_{0}$, consisting of surfaces whose area is equal that of $x_{0}$. (In the notation of $\underline{\mathrm{KoZO}}$, when $\mathbf{g} \geqslant 3$ is odd, the connected component of $x_{0}$ is $\mathcal{H}(\mathbf{g}-$ $1, \mathbf{g}-1)^{\text {odd }}$ and when $\mathbf{g} \geqslant 4$ is even, it is $\mathcal{H}(\mathbf{g}-1, \mathbf{g}-1)^{\text {non-hyp }}$. This follows from Proposition 3.3.)

Theorem 1.4. For $\mathbf{g} \geqslant 3$, the rel leaf of $x_{0}$ is dense in $\mathcal{H}_{0}$.

Theorem 1.4 gives the first explicit examples of dense rel leaves in these strata.

In case $\mathbf{g}=2$ the analogue of the Arnoux-Yoccoz surface was defined by Bowman in the appendix of [Bow1. It is a disconnected surface (more accurately, two tori attached at a node) in $\mathcal{H}(0) \times \mathcal{H}(0)$, which can be thought of as part of a boundary of the genus two stratum $\mathcal{H}(1,1)$ (see [B] ). In fact, in the terminology of McMullen [McM2], it 
is an eigenform surface for discriminant $D=5$. Although it does not make sense to discuss the rel leaf of $x_{0}$, one can view it as a terminal point of an imaginary rel leaf in the $D=5$ eigenform locus $\mathcal{E}_{5}(1,1) \subset$ $\mathcal{H}(1,1)$. Using this point of view both of our main results extend to $\mathrm{g}=2$ as follows:

Theorem 1.5. When $\mathbf{g}=2$ there is a surface $x_{1} \in \mathcal{E}_{5}(1,1)$, such that surfaces $x_{s}=\operatorname{Rel}_{s-1}^{(v)} x_{1}$ have the following properties:

(i) The surfaces $x_{s}$ are defined for all $s>0$.

(ii) The surfaces $x_{s}$ are horizontally periodic, and as $s \rightarrow+\infty$, the circumferences of the horizontal cylinders tend uniformly to 0.

(iii) In a suitable compactification of $\mathcal{H}(1,1)$, the limit $x_{0}=\lim _{s \rightarrow 0+} x_{s}$ exists and is the "genus 2 Arnoux-Yoccoz surface" of [Bow1].

(iv) The rel-leaf of $x_{1}$ is dense in $\mathcal{E}_{5}(1,1)$.

\subsection{Related ideas and works.}

1.1.1. Linear deformations of IETs. Theorem 1.1 has implications for the study of unique ergodicity of interval exchange transformations (IETs), which we now describe. Let $\mathbb{R}_{+}^{d}$ denote the vectors in $\mathbb{R}^{d}$ with positive entries. For a fixed permutation $\sigma$ on $d$ symbols, and $\mathbf{a}=$ $\left(a_{1}, \ldots, a_{d}\right) \in \mathbb{R}_{+}^{d}$, let $\mathcal{I} \mathcal{E}=\mathcal{I E}_{\sigma}(\mathbf{a}): I \rightarrow I$ be the interval exchange transformation obtained by partitioning the interval $I=\left[0, \sum a_{i}\right)$ into subintervals of lengths $a_{1}, \ldots, a_{d}$ and permuting them according to $\sigma$. Assuming irreducibility of $\sigma$, it is known that for almost all choices of $\mathbf{a}, \mathcal{I E}_{\sigma}(\mathbf{a})$ is uniquely ergodic, but nevertheless that the set of nonuniquely ergodic interval exchanges is not very small (see [MaTa] for definitions, and a survey of this intensively studied topic). It is natural to expect that all line segments in $\mathbb{R}_{+}^{d}$, other than some obvious counterexamples, should inherit the prevalence of uniquely ergodic interval exchanges. A conjecture in this regard was made by the second-named author in [W, Conj. 2.2], and partial positive results supporting the conjecture were obtained in [MW, Thm. 6.1]. Namely, a special case of [MW, Thm. 6.1] asserts that for any uniquely ergodic $\mathcal{I} \mathcal{E}_{0}=\mathcal{I E}_{\sigma}\left(\mathbf{a}_{0}\right)$, there is an explicitly given hyperplane $\mathcal{L} \subset \mathbb{R}_{+}^{d}$ containing $\mathbf{a}_{0}$, such that for any line segment $\ell=\left\{\mathbf{a}_{s}: s \in I\right\} \subset \mathbb{R}_{+}^{d}$ with $\ell \varnothing \mathcal{L}$, there is an interval $I_{0} \subset I$ containing 0 , such that for almost every $s \in I_{0}, \mathcal{I E}_{\sigma}\left(\mathbf{a}_{s}\right)$ is uniquely ergodic.

This expectation is false. In fact counterexamples to [W, Conj. 2.2] were exhibited by Dynnikov ${ }^{1}[\mathrm{D1}$, and further related results were obtained in the subsequent work [DD, DS, AHS]. In particular it was

\footnotetext{
${ }^{1}$ Dynnikov's counterexamples appeared (in a different context and using different terminology) three years before [W, Conj. 2.2] was formulated. However the
} 
shown that there are linear subspaces $\mathcal{L} \subset \mathbb{R}^{d}$ such that almost every point in $\mathcal{L}$ is non-minimal, and yet $\mathcal{L}$ contains an uncountable closed set of uniquely ergodic points. Our results can be used to give a stronger refutation of [W, Conj. 2.2]. Recall that a standard construction of an interval exchange transformation, is to fix a translation surface $q$ with a segment $\gamma$ transverse to horizontal lines, and define $\mathcal{I E}(q, \gamma)$ to be the first return map to $\gamma$ along horizontal leaves on $q$ (where we parameterize $\gamma$ using the transverse measure $d y)$. If $L=\{x(s)$ : $s \in I\}$ is a sufficiently small straight line segment in a stratum of translations surfaces, $\gamma$ can be chosen uniformly for all $s \in I$ and the interval exchanges $\mathcal{I E}(x(s), \gamma)$ can be written as $\mathcal{I E}_{\sigma}(\mathbf{a}(s))$ for some fixed permutation $\sigma$ and some line $\ell=\{\mathbf{a}(s): s \in I\} \subset \mathbb{R}_{+}^{d}$ (see [MW] for more details). The number of intervals one obtains for surfaces in

$\mathcal{H}(\mathbf{g}-1, \mathbf{g}-1)$ is $2 \mathbf{g}+1$. Thus, taking $x(s)=\operatorname{Rel}_{s}^{(v)} x_{0}$, Corollary 1.2 implies:

Corollary 1.6. For $d=7$, there is a uniquely ergodic self-similar interval exchange transformation $\mathcal{I E}_{0}$ on d intervals, and a line segment $\ell \subset \mathbb{R}_{+}^{d}$ such that $\mathcal{I} \mathcal{E}_{0}=\mathcal{I} \mathcal{E}_{\sigma}\left(\mathbf{a}_{0}\right)$ with $\mathbf{a}_{0}$ in the interior of $\ell$, and such that for all $\mathbf{a} \in \ell \backslash\left\{\mathbf{a}_{0}\right\}, \mathcal{I E}_{\sigma}(\mathbf{a})$ is periodic.

1.1.2. Rel leaves. It is well-known that some imaginary-rel trajectories exit their stratum in finite time due to the collapse of vertical saddle connections. In this case the imaginary-rel trajectory is not defined for all time. Theorem 1.1 shows that an imaginary-rel trajectory may be defined for all time and still be divergent in its stratum. This should be contrasted with the horocycle flow for which there are no divergent trajectories (see [V2]).

Although we only discuss the rel leaves of the Arnoux-Yoccoz surfaces in this paper, it is quite likely that the rel foliation is ergodic in any stratum with more than one singularity, and thus almost all rel leaves are dense in all such strata. Recently Calsamiglia, Deroin and Francaviglia [CDF] and Hamenstädt [H] have proved ergodicity in principal strata, i.e. strata where all singularities are simple. The methods of proof used in these papers are very different from the one used in this paper.

Individual rel leaves have a natural translation structure (see McM5, MW]). In [McM5, Thm. 1.1] it was shown that in a suitable bordification of the stratum, this geometric structure is complete (note that the conventions used in McM5 differ from those used in this paper

authors only became aware of this after a preliminary version of this paper was circulated. 
by taking the quotient by a finite permutation group, e.g. if $k=2$ then with our conventions rel leaves are translation surfaces and with McMullen's, they are half-translation surfaces). Furthermore, in genus 2, results of McMullen [McM3, McM5] give a detailed understanding of the closure of rel leaves in the eigenform locus. These results should be viewed as a companion to McMullen's celebrated result classifying closed sets invariant under the action of $G=\mathrm{SL}_{2}(\mathbb{R})$ in genus 2 [McM2].

1.1.3. The Arnoux-Yoccoz IET and the SAF invariant. This work began with computer experiments in the case $\mathbf{g}=3$ which demonstrated the complete periodicity of the vertical foliation on real-rel deformations of $x_{0}$. These computer experiments were suggested by Michael Boshernitzan, and the observed results are now described by Corollary 1.2 .

These experiments were in turn motivated by several related observations. First, the Arnoux-Yoccoz IETs have vanishing SAF invariant. This is evident from their construction in [AY], and follows from the various functorial properties of the SAF invariant; see [Arn2, §IV] or [HL1, §2.1]. These properties of the SAF invariant also imply that the invariant is well-defined for the vertical foliation on any translation surface suspension of the IET, and is rel-invariant. Thus every rel deformation of $x_{0}$ has vanishing SAF invariant. A vanishing SAF invariant is often associated with a completely periodic IET, so it was natural to wonder if rel deformations of $x_{0}$ are often vertically completely periodic; and a precursor to this work [HW] investigated this question.

1.1.4. Algebraic dynamics. For any interval exchange map $T_{0}:[0,1] \rightarrow$ $[0,1]$, observe that the displacements $T_{0}(x)-x$ lie in a finitely generated subgroup of $\mathbb{R} / \mathbb{Z}$. One can then consider the Cayley graph of this group where the generators are taken to be the possible values of $T_{0}(x)-x$. Any $T_{0}$-orbit then gives rise to a parameterized path $n \mapsto T_{0}^{n}(x)-x$ in this Cayley graph. The SAF-invariant may be interpreted as the average displacement in this subgroup, so vanishing SAF-invariant is suggestive of prevalent closed paths or paths which diverge sublinearly (though other behavior is possible). This makes these paths particularly interesting when the SAF-invariant vanishes.

The discontinuity points of an IET $T_{0}$ have a natural partition into subsets corresponding to singular points of any suspension of the IET, and a perturbation $T_{t}$ of the IET in which all discontinuity points corresponding to one singular point are moved by the same $t \in \mathbb{R}$ (where $t$ is small enough so that no interval collapses) corresponds to a rel deformation. Such perturbations change the IET without affecting 
the group generated by the displacements, so the Cayley graph above remains invariant. Thus it is interesting to consider how the paths change as we vary $t$. Note that the paths that arise vary continuously in $t$ where we use the topology of pointwise convergence on paths viewed as functions from $\mathbb{Z}$ to the Cayley graph.

In [LPV], a variant these paths were studied for the Arnoux-Yoccoz IET $\mathcal{I} \mathcal{E}_{0}$. In this case the group generated by possible displacements (modulo 1 ) is isomorphic to $\mathbb{Z}^{2}$ and the Cayley graph is isomorphic to the hexagonal tiling of the plane. The article [LPV] found combinatorial space filling curves for $\mathcal{I} \mathcal{E}_{0}$. The paths for $\mathcal{I} \mathcal{E}_{0}$ were also discussed in [McM6, §5] as well as paths for other IETs. A closed path was found in [McM6, end of §5] associated to an IET arising from one small reldeformation of the Arnoux-Yoccoz surface of genus 3. We find that under rel deformations, the closed combinatorial orbits associated to the closed orbits of $\mathcal{I} \mathcal{E}_{t}$ fill larger and larger regions in the group as $t \rightarrow 0$; see Figure 2 and the further discussion in $\$ 4.3$. This phenomenon may be viewed as a consequence of continuity and the space filling results in [LPV].

This sort of algebraic dynamics does not seem to have been used heavily in the study of IETs except in the case when the subgroup of $\mathbb{R} / \mathbb{Z}$ mentioned above is isomorphic a finite extension of $\mathbb{Z}$. This case was considered by Arnoux [Arn2, §IV.8], Boshernitzan [Bo] and McMullen [McM1] and plays a role in the result that ergodic IETs in this case have non-vanishing SAF-invariant.

1.1.5. The work of Dynnikov. We remark that the Arnoux-Yoccoz surface in genus $\mathbf{g}=3$ is a suspension of the Arnoux-Yoccoz IET, which belongs to a family of IETs parameterized by three real numbers whose interval lengths vary linearly in the parameters and can be understood through methods of Dynnikov [D2] which differ greatly from the approach of this article. Lebesgue almost every IET in the family is nonminimal, and there is a renormalization scheme which can be used to understand these IETs. In particular, a phenomenon similar to Corollary 1.2 is exhibited in [D1, §7], where complete periodicity holds in a parameter neighborhood of a minimal IET.

1.1.6. $\mathrm{SL}_{2}(\mathbb{R})$-orbit closures. Let $G=\mathrm{SL}_{2}(\mathbb{R})$ and let $U, V \subset G$ be the unipotent subgroups which shear in the horizontal and vertical directions respectively. A recent breakthrough of Eskin, Mirzakhani and Mohammadi [EMiMo] describes the $G$-invariant closed subsets for arbitrary strata. The following result, which we deduce from their work, is important for our proof of Theorem 1.4, but should also be of independent interest: 


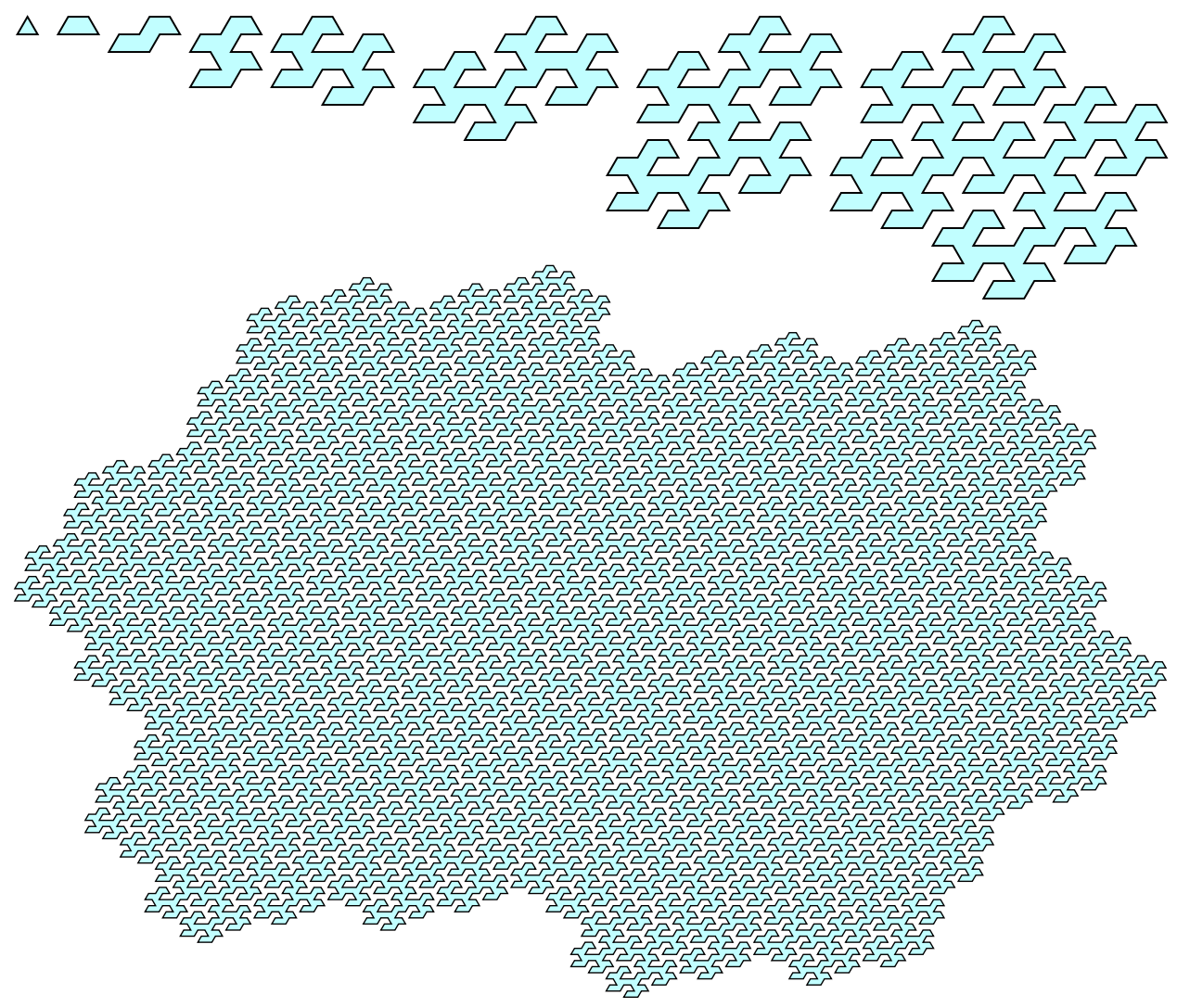

FiguRE 2. Depictions of the algebraic dynamics of periodic orbits of the rel deformed Arnoux-Yoccoz IET in genus three. The 1st through 8th and 15th shortest combinatorial orbits are shown. See 44.3 for more detail.

Proposition 1.7. Suppose that $x$ is a translation surface and $\left\{g_{t} x\right.$ : $t \in \mathbb{R}\}$ is a periodic trajectory for the geodesic flow. Then the orbit closures $\overline{U x}, \overline{V x}$ and $\overline{G x}$ coincide.

The proof makes use of results of [EMiMo] on the orbit closures under the upper-triangular subgroup of $G$.

In case $\mathbf{g}=3$, Hubert, Lanneau and Möller [HLM] determined $\overline{G x_{0}}$, proving that it coincides with the set of surfaces in $\mathcal{H}_{0}$ admitting a hyperelliptic involution. In case $\mathbf{g} \geqslant 4$, we use an idea of Alex Wright to show that $\overline{G x_{0}}$ is an affine manifold of full rank (see $\$ 5.2$ ). The argument requires the following algebraic fact, which was proved in response to our question (see Appendix A, Corollary A.8): 
Theorem 1.8 (Bary-Soroker, Shusterman and Zannier). Let $\mathbf{g} \geqslant 3$ and let $\alpha$ be the unique real number in $[0,1]$ satisfying $\alpha+\cdots+\alpha \mathbf{g}=1$. Then $\mathbb{Q}(\alpha)$ contains no totally real subfields other than $\mathbb{Q}$.

The following result is of independent interest and follows from Theorem 1.8 and a recent preprint of Mirzakhani and Wright [MiWr], via an argument of Alex Wright. It could be used to simplify some of the steps in the proof of Theorem 1.4, see Remark 5.6.

Theorem 1.9. If $\mathbf{g} \geqslant 4$, then $\overline{G x_{0}}=\mathcal{H}_{0}$.

1.1.7. Twist coordinates for horizontally periodic surfaces. Let $x$ be a horizontally completely periodic translation surface in the stratum $\mathcal{H}$. Observe that surfaces in the horocycle orbit $U x$ all have a horizontal cylinder decomposition with the same circumferences, same cylinder widths, and same lengths of horizontal saddle connections. By deforming $x$ (in any manner) while leaving these quantities constant, a subset of $\mathcal{H}$ is obtained which is naturally the image of a torus, and twist coordinates can be placed on this torus. In [SW], it was shown that the horocycle dynamics on the orbit closure $\overline{U x}$ can be described as linear flow on this torus in twist coordinates, and this observation was used to describe horocycle orbit closures of horizontally completely periodic surfaces.

In this article we observe that twist coordinates can also be used to describe real rel orbits of $x$, and the dynamics are again linear flow on a torus. In these coordinates, the horocycle flow orbit-closure is determined by the moduli of the cylinders while the real-rel orbit-closure is determined by their circumferences. We use this here to show the horocycle orbit of the Arnoux-Yoccoz surface $x_{0}$ is contained in the closure of the rel leaf of $x_{0}$.

1.2. Outline of paper and proofs. We now discuss the proofs of our results.

Sections 3 and 4 contain the proof of Theorem 1.1 (as well as Theorem 1.5 and Corollary 1.2. We obtain a description of surfaces $x_{t}=\operatorname{Rel}_{t}^{(v)} x_{0}$ indirectly. Section 3 concentrates on studying a mystery imaginary rel trajectory, and $\$ 4$ shows it is the imaginary rel trajectory of the Arnoux-Yoccoz surface.

- In $\$ 3.1$ we review the definition of the Arnoux-Yoccoz interval exchange $\mathcal{I E}$ on $2 \mathrm{~g}+1$ intervals. Theorem 3.1 describes a renormalizing symmetry of $\mathcal{I} \mathcal{E}$ known to Arnoux and Yoccoz. We prove this result for completeness.

- In $\$ 3.2$ we discuss suspensions of $\mathcal{I E}$, all of which lie in the stratum $\mathcal{H}(\mathbf{g}-1, \mathbf{g}-1)$. We find the connected component of the stratum 
containing suspensions of $\mathcal{I E}$ in Proposition 3.3. We associate to the renormalization of $\mathcal{I E}$ a canonical mapping class $[\phi]$ in Corollary 3.4 and Definition 3.5 .

- In $\$ 3.3$, we choose a special suspension $q_{0} \in \mathcal{H}(\mathbf{g}-1, \mathbf{g}-1)$ of $\mathcal{I E}$ which is horizontally completely periodic. The imaginary rel trajectory is denoted $q_{s}=\operatorname{Rel}_{s}^{(v)} q$ whenever defined. Theorem 3.7 shows that these surfaces exhibit a useful symmetry (which explains our choice of $q_{0}$ ): After reparameterizing the trajectory as $x_{t}=q_{t-\beta}$ for $\beta=\frac{\alpha^{2}}{1-\alpha}$ we observe the coincidence $\tilde{g} x_{\alpha^{-1} t}=x_{t}$ for all $t$, where $\tilde{g} \in \mathrm{SL}(2, \mathbb{R})$ is a diagonal matrix expanding the vertical direction by a factor of $\alpha$. Furthermore, the mapping class determined by deforming $x_{t}$ into $x_{\alpha^{-1} t}$ by applying vertical rel and then applying $\tilde{g}$ to get back to $x_{t}$ is $[\phi]$ mentioned above. We show all this by explicit computations, and along the way demonstrate that the surfaces $x_{t}$ for $t>0$ are all horizontally completely periodic. From the symmetry above, it suffices to show $x_{t}$ is horizontally completely periodic for $t$ taken from the fundamental domain $[\beta, \beta / \alpha]$ for the $\tilde{g}$ action on the vertical rel ray.

- In $\$ 3.4$, we prove a lemma about the horizontal cylinders of $x_{t}$ for $t>0$. This will be used later to understand how twist coordinates change when we apply the real rel flow to $x_{t}$ for $t>0$, which is an ingredient in the proof that the full rel leaf is dense in the sense of Theorem 1.4.

- We consider the case of $\mathbf{g} \geqslant 3$ in 44.1 . Lemma 4.1 shows that in this case the mapping class $[\phi]$ is pseudo-Anosov. We argue that suspensions of $\mathcal{I E}$ have infinite vertical rel trajectories, and thus there is a surface $x_{0}$ so that $\tilde{g} x_{0}=x_{0}$. Theorem 4.3 uses Thurston's theory of pseudo-Anosov homeomorphisms of surfaces to argue that $x_{0}$ must be the Arnoux-Yoccoz surface. This completes the proof of Theorem 1.1.

- In $\$ 4.2$ we discuss the case of $\mathbf{g}=2$ and prove Theorem 1.5 .

- In $\$ 4.3$ we describe additional symmetries that show up when $\mathbf{g}=3$ and prove Corollary 1.2. We note that most of these additional symmetries do not persist when $\mathbf{g} \geqslant 4$. We discuss the arithmetic dynamics in the case of $\mathbf{g}=3$ as mentioned above.

For the proof of Theorem 1.4, we successively show that the closure of the rel leaf of the Arnoux-Yoccoz surface $x_{0}$ is larger and larger. From this point of view, there are two steps, which we undertake in reverse order. In $\$ 6$ we show that the orbit $U x_{0}$ is in this closure. In $\$ 5$, we show that it follows that the closure of the rel leaf is the stratum 
connected component $\mathcal{H}_{0}$. Here is a more detailed description of these sections.

Section 5 reviews the relevant results from Eskin, Mirzakhani and

Mohammadi, and then discusses consequences used in the proof of Theorem 1.4.

- In $\$ 5.1$ we prove Proposition 1.7 .

- After reviewing results of Filip and Wright, along with some ideas due to Wright, $\$ 5.2$ culminates in the observation that when $\mathbf{g} \geqslant 3$ the only closed subset of $\mathcal{H}_{0}$ which is rel-invariant and contains $U x_{0}$ is $\mathcal{H}_{0}$; see Corollary 5.8. This means that in order to show the closure of the rel leaf contains $\mathcal{H}_{0}$ it suffices to show that this closure contains the horocycle orbit $U x_{0}$.

In $\$ 6$ we analyze the interaction of real-rel and the horocycle flow on surfaces which have a decomposition into parallel horizontal cylinders.

- It 6.1 we consider deforming a horizontally completely periodic surface while preserving cylinder dimensions and horizontal saddle connection lengths. We describe how to place twist coordinates on this deformation space and observe that the subset of the stratum obtained in this way is an orbifold quotient of a torus.

- In $\$ 6.2$ we consider the real rel-flow applied to a horizontally completely periodic translation surface with no horizontal saddle connections joining distinct singularities. We find that in twist coordinates, the real rel-flow can be explicitly described as linear flow on a torus (or its quotient as above).

- In 86.3 we specialize the results of the preceding subsections to the case of the horizontally periodic surfaces $x_{s}=\operatorname{Rel}_{s}^{(v)} x_{0}$ where $x_{0}$ is the Arnoux-Yoccoz surface. By taking closures of real real deformations of such surfaces $x_{s}$, we find a family of tori $\left\{\mathcal{O}_{s}\right\}$ of dimension greater than 1, defined for all but a discrete set of $s>0$. Our explicit parameterization enables us to compute the behavior of the tangent planes $T_{s}$ to $\mathcal{O}_{s}$, as $s \rightarrow 0+$. Recalling that twist coordinates can also be used to describe horizontal horocycle orbits (following SW]), we show that the topological limit of the $\mathcal{O}_{s}$ contains the entire horocycle orbit $U x_{0}$, concluding the proof.

The appendix by Bary-Soroker, Shusterman and Zannier contains the proof of Theorem 1.8 (which is crucial to our arguments in 55.2 .)

1.3. Acknowledgments. This work was stimulated by insightful comments of Michael Boshernitzan, who conjectured Corollary 1.6. We thank Alex Wright for directing our attention to the case $\mathbf{g}=2$ and 
for his proofs of Theorems 1.9 and 5.3. Theorem 1.8, which is a crucial step in our proof of Theorem 1.4, was proved in response to our queries by Lior Bary-Soroker, Mark Shusterman, and Umberto Zannier. We thank them for agreeing to include their results in Appendix A of this paper. We thank Ivan Dynnikov, Pascal Hubert and Sasha Skripchenko for pointing out the connections to their prior work and other insightful remarks. We are also grateful to David Aulicino, Josh Bowman, Duc-Manh Nguyen and John Smillie for useful discussions. We also are grateful to the anonymous referee for useful comments which helped to improve the paper. This collaboration was supported by BSF grant 2010428. The first author's work is supported by NSF grant DMS-1500965 as well as a PSC-CUNY Award (funded by The Professional Staff Congress and The City University of New York). The second author's work was supported by ERC starter grant DLGAPS 279893.

\section{BASICS}

2.1. Translation surfaces, strata, $G$-action, cylinders. In this section we define our objects of study and review their basic properties. We refer to [MaTa, Wr2] and [Zo] for more information on translation surfaces and related notions, and for references for the statements given in this subsection.

Let $S$ be a compact oriented surface of genus $\mathbf{g} \geqslant 2$, let $\Sigma=$ $\left\{\xi_{1}, \ldots, \xi_{k}\right\} \subset S$ be a finite set of points and let $\mathbf{r}=\left(r_{1}, \ldots, r_{k}\right)$ be a list of non-negative integers such that $\sum r_{i}=2 \mathrm{~g}-2$. A translation atlas of type $\mathbf{r}$ on $(S, \Sigma)$ is an atlas of charts $\left(U_{\alpha}, \varphi_{\alpha}\right)$, where:

- For each $\alpha$, the set $U_{\alpha} \subset S \backslash \Sigma$ is open, and the map

$$
\varphi_{\alpha}: U_{\alpha} \rightarrow \mathbb{R}^{2}
$$

is continuous and injective.

- The union over $\alpha$ of $U_{\alpha}$ is $S \backslash \Sigma$.

- Whenever the sets $U_{\alpha}$ and $U_{\beta}$ intersect, the transition functions are local translations, i.e., the maps

$$
\varphi_{\beta} \circ \varphi_{\alpha}^{-1}: \varphi_{\alpha}\left(U_{\alpha} \cap U_{\beta}\right) \rightarrow \mathbb{R}^{2}
$$

are differentiable with derivative equal to the identity.

- Around each $\xi_{j} \in \Sigma$ the charts glue together to form a cone singularity with cone angle $2 \pi\left(r_{j}+1\right)$.

A translation surface structure on $(S, \Sigma)$ of type $\mathbf{r}$ is an equivalence class of such translation atlases, where $\left(U_{\alpha}, \varphi_{\alpha}\right)$ and $\left(U_{\beta}^{\prime}, \varphi_{\beta}^{\prime}\right)$ are equivalent if there is an orientation preserving homeomorphism $h: S \rightarrow S$, fixing all points of $\Sigma$, such that $\left(U_{\alpha}, \varphi_{\alpha}\right)$ is compatible with $\left(h\left(U_{\beta}^{\prime}\right), \varphi_{\beta}^{\prime} \circ h^{-1}\right)$. 
A marked translation surface structure is an equivalence class of such atlases subject to the finer equivalence relation where $\left(U_{\alpha}, \varphi_{\alpha}\right)$ and $\left(U_{\beta}^{\prime}, \varphi_{\beta}^{\prime}\right)$ are equivalent if $h$ can be taken to be isotopic to the identity via an isotopy fixing $\Sigma$. Thus, a marked translation surface $\mathbf{q}$ determines a translation surface $q$ by forgetting the marking, and we write $q=\pi(\mathbf{q})$ to denote this operation. Note that our convention is that all singularities are labeled.

Pulling back $d x$ and $d y$ from the coordinate charts we obtain two well-defined closed 1-forms, which we can integrate along any path $\gamma$ on $S$. If $\gamma$ is a cycle or has endpoints in $\Sigma$ (a relative cycle), then we define

$$
\operatorname{hol}_{\mathrm{x}}(\gamma, \mathbf{q})=\int_{\gamma} d x \text { and } \operatorname{hol}_{\mathrm{y}}(\gamma, \mathbf{q})=\int_{\gamma} d y
$$

These integrals only depend on the homology class of $\gamma$ in $H_{1}(S, \Sigma)$ and the pair of these integrals is the holonomy of $\gamma$,

$$
\operatorname{hol}(\gamma, \mathbf{q})=\left(\begin{array}{l}
\operatorname{hol}_{\mathrm{x}}(\gamma, \mathbf{q}) \\
\operatorname{hol}_{\mathrm{y}}(\gamma, \mathbf{q})
\end{array}\right) \in \mathbb{R}^{2}
$$

We let $\operatorname{hol}(\mathbf{q})=\operatorname{hol}(\cdot, \mathbf{q})$ be the corresponding element of $H^{1}\left(S, \Sigma ; \mathbb{R}^{2}\right)$, with coordinates $\mathrm{x}(\mathbf{q})$ and $\mathrm{y}(\mathbf{q})$ in $H^{1}(S, \Sigma ; \mathbb{R})$.

The set of all (marked) translation surfaces on $(S, \Sigma)$ of type $\mathbf{r}$ is called the stratum of (marked) translation surface of type $\mathbf{r}$ and is denoted by $\mathcal{H}(\mathbf{r})$ (resp. $\mathcal{H}_{\mathrm{m}}(\mathbf{r})$ ). The map hol : $\mathcal{H}_{\mathrm{m}}(\mathbf{r}) \rightarrow H^{1}\left(S, \Sigma ; \mathbb{R}^{2}\right)$ just defined gives local charts for $\mathcal{H}_{\mathrm{m}}(\mathbf{r})$, endowing it $(\operatorname{resp} . \mathcal{H}(\mathbf{r}))$ with the structure of an affine manifold (resp. orbifold).

Let $\operatorname{Mod}(S, \Sigma)$ denote the mapping class group, i.e. the orientation preserving homeomorphisms of $S$ fixing $\Sigma$ pointwise, up to an isotopy fixing $\Sigma$. The map hol is $\operatorname{Mod}(S, \Sigma)$-equivariant. The $\operatorname{Mod}(S, \Sigma)$-action on $\mathcal{H}_{\mathrm{m}}$ is properly discontinuous. Thus $\mathcal{H}(\mathbf{r})=\mathcal{H}_{\mathrm{m}}(\mathbf{r}) / \operatorname{Mod}(S, \Sigma)$ is a linear orbifold and $\pi: \mathcal{H}_{\mathrm{m}}(\mathbf{r}) \rightarrow \mathcal{H}(\mathbf{r})$ is an orbifold covering map. In particular, if $\left\{\mathbf{q}_{s}: s \in[0,1]\right\}$ is a continuous path in $\mathcal{H}_{\mathrm{m}}$ with $\pi\left(\mathbf{q}_{0}\right)=\pi\left(\mathbf{q}_{1}\right)$ then there is a corresponding element of $\operatorname{Mod}(S, \Sigma)$ which maps $\mathbf{q}_{0}$ to $\mathbf{q}_{1}$ and is independent of the choice of the lift. We have

$$
\operatorname{dim} \mathcal{H}(\mathbf{r})=\operatorname{dim} \mathcal{H}_{\mathrm{m}}(\mathbf{r})=\operatorname{dim} H^{1}\left(S, \Sigma ; \mathbb{R}^{2}\right)=2(2 g+k-1) .
$$

There is an action of $G=\mathrm{SL}_{2}(\mathbb{R})$ on $\mathcal{H}(\mathbf{r})$ and on $\mathcal{H}_{\mathrm{m}}(\mathbf{r})$ by postcomposition on each chart in an atlas. The projection $\pi: \mathcal{H}_{\mathrm{m}}(\mathbf{r}) \rightarrow$ $\mathcal{H}(\mathbf{r})$ is $G$-equivariant. The $G$-action is linear in the homology coordinates, namely, given a marked translation surface structure $\mathbf{q}$ and $\gamma \in H_{1}(S, \Sigma)$, and given $g \in G$, we have

$$
\operatorname{hol}(\gamma, g \mathbf{q})=g \cdot \operatorname{hol}(\gamma, \mathbf{q}) \text {, }
$$


where on the right hand side, $g$ acts on $\mathbb{R}^{2}$ by matrix multiplication. We will write

$$
u_{s}=\left(\begin{array}{cc}
1 & s \\
0 & 1
\end{array}\right), \quad g_{t}=\left(\begin{array}{cc}
e^{t} & 0 \\
0 & e^{-t}
\end{array}\right), \quad v_{s}=\left(\begin{array}{cc}
1 & 0 \\
s & 1
\end{array}\right) .
$$

Also we will denote

$$
\begin{gathered}
U=\left\{u_{s}: s \in \mathbb{R}\right\}, \quad A=\left\{g_{t}: t \in \mathbb{R}\right\}, \\
V=\left\{v_{s}: s \in \mathbb{R}\right\}, \quad P=A U=\left(\begin{array}{cc}
* & * \\
0 & *
\end{array}\right) \subset G .
\end{gathered}
$$

The connected components of strata $\mathcal{H}(\mathbf{r})$ have been classified by Kontsevich and Zorich [KoZ0]. We will be interested in the particular connected component of $\mathcal{H}(\mathbf{g}-1, \mathbf{g}-1)$ containing $x_{0}$. For any $\mathbf{r}$, the area of surfaces in $\mathcal{H}(\mathbf{r})$ is preserved by the action of $G$ and we let $\mathcal{H}$ be a fixed-area sub-locus of a connected component of $\mathcal{H}(\mathbf{r})$. A common convention is to normalize area by setting $\mathcal{H}$ to be the locus of area-one surfaces, but it will be more convenient for us to fix the area equal to some constant, e.g. the area of $x_{0}$. There is a globally supported measure on $\mathcal{H}$ which is defined using Lebesgue measure on $H^{1}\left(S, \Sigma ; \mathbb{R}^{2}\right)$ and a 'cone construction'. It was shown by Masur that the $G$-action is ergodic with respect to this measure, and in particular, almost every $G$-orbit is dense.

Let $\vec{u} \in \mathbb{R}^{2}$ be a unit vector. The straight-line flow in direction $\vec{u}$ on a translation surface $q$ is the flow $F_{t}: q \rightarrow q$ defined so that holonomy of a trajectory of length $t$,

$$
[0, t] \rightarrow q ; \quad s \mapsto F_{s}(p),
$$

is $t \vec{u}$. Because of the cone singularities, the straight-line flow does not need to be defined for all time. The straight-line flow in direction $(0,1)$ will be called the vertical straight-line flow.

A separatrix on a translation surface is a straight-line trajectory leaving a singularity. A saddle connection is a straight-line trajectory terminating at singularities in forward and backward time.

An affine automorphism of a translation surface $q$ is a homeomorphism of $q$ which leaves invariant the set of singular points and which is affine in charts. The derivative of an affine automorphism is a $2 \times 2$ real matrix of determinant \pm 1 . If this matrix is hyperbolic (i.e. has distinct real eigenvalues) then the affine automorphism is called a pseudoAnosov map. If the matrix is parabolic (i.e. is nontrivial and has both eigenvalues equal to 1 ) then the affine automorphism is called parabolic. The group of derivatives of orientation preserving affine automorphisms of $q$ is called the Veech group of $q$. 
The field $k$ generated over $\mathbb{Q}$ by the traces of all derivatives of pseudoAnosov maps of $q$ is called the trace field of $q$. In fact, by a theorem of Kenyon and Smillie [KS], $k$ is generated by the trace of any single pseudo-Anosov map of $q$. The holonomy field of $q$ is the smallest field $k$ such that there is $g \in \mathrm{GL}_{2}(\mathbb{R})$ and a marked surface $\mathbf{q}$ with $\pi(\mathbf{q})=q$ such that for all $\gamma \in H_{1}(S ; \mathbb{Z}), g \cdot \operatorname{hol}(\gamma, \mathbf{q}) \in k^{2}$. In $[\mathrm{KS}$ ] it is shown that if $q$ has a pseudo-Anosov map then the holonomy field coincides with the trace field.

Let $I \subset \mathbb{R}$ be a closed interval with interior, let $c>0$ and let $\mathbb{R} / c \mathbb{Z}$ be the circle of circumference $c$. A cylinder on a translation surface is a subset homeomorphic to an annulus which is the image of $I \times \mathbb{R} / c \mathbb{Z}$ for some $I$ and $c$ as above, under a map which is a local Euclidean isometry, and which is maximal in the sense that the local isometry does not extend to $J \times \mathbb{R} / c \mathbb{Z}$ for an interval $J$ properly containing $I$. The parameter $c$ is called the circumference of the cylinder, and the image of $\{t\} \times \mathbb{R} / c \mathbb{Z}$ for some $t \in \operatorname{int}(I)$ is called a core curve. In this case the two boundary components of the cylinder are unions of saddle connections whose holonomies are all parallel to that of the core curve. If a translation surface $q$ can be represented as a union of cylinders, which intersect along their boundaries, then the directions of the holonomies of the core curves of the cylinders are all the same, and we say that this direction is completely periodic and that $q$ has a cylinder decomposition in that direction.

If $C$ is a cylinder with an oriented core curve on a translation surface modeled on $S$, we use $C^{*}$ to denote the associated cohomology class in $H^{1}(S, \Sigma ; \mathbb{Z})$ defined by

$$
C^{*}(\gamma)=\gamma \cap C \quad \text { for } \gamma \in H_{1}(S, \Sigma ; \mathbb{Z}),
$$

where $\cap$ denotes the algebraic intersection pairing between $\gamma$ and the oriented core curve. Recall that the intersection pairing is nondegenerate as a bilinear form on $H_{1}(S, \Sigma) \times H_{1}(S \backslash \Sigma)$.

2.2. Interval exchange maps, suspensions, and pseudo-Anosov maps. We define interval exchange tranformations (IETs) on the circle $\mathbb{T}=\mathbb{R} / \mathbb{Z}$ rather than the interval, because the original Arnoux-Yoccoz IET was defined on the circle. An interval exchange transformation of the circle is a bijective piecewise rotation

$$
\mathcal{I E}: \mathbb{T} \rightarrow \mathbb{T} ; \quad \mathcal{I E}(x)=x+t_{j} \quad \text { for } x \in I_{j},
$$

where $\left\{I_{j}=\left[a_{j}, b_{j}\right)+\mathbb{Z}\right\}$ is a partition of $\mathbb{T}$ into finitely many half open intervals, each $t_{j}$ is in $\mathbb{T}$, and addition is considered $\bmod \mathbb{Z}$. Then each restriction $\left.\mathcal{I E}\right|_{I_{j}}$ rotates $I_{j}$ by $t_{j}$. We call the endpoints of the intervals $I_{j}$ the discontinuities of $\mathcal{I} \mathcal{E}$. 
A suspension of an interval exchange map $\mathcal{I E}: \mathbb{T} \rightarrow \mathbb{T}$ is a pair $(q, \gamma)$, where $q$ is a translation surface and $\gamma: \mathbb{T} \rightarrow q \backslash \Sigma$ is a parameterized simple closed curve which transversely passes rightward over the vertical foliation of $q$ such that (informally) $\gamma$ conjugates $\mathcal{I E}$ to the return map of the vertical straight-line flow to $\gamma(\mathbb{T})$. Formally, we insist:

- If $x \in \mathbb{T}$ is not a discontinuity, then the vertical straight-line trajectory of $\gamma(x)$ hits $\gamma \circ \mathcal{I E}(x)$ before hitting any singularities and before hitting any other point on $\gamma(\mathbb{T})$.

- If $x \in \mathbb{T}$ is a discontinuity, then $\gamma(x)$ hits a singularity under the vertical straight-line flow.

We call such a suspension measure preserving if $\gamma$ sends Lebesgue measure on $\mathbb{T}$ to the measure on $\gamma(\mathbb{T})$ induced by the Lebesgue transverse measure to the vertical foliation on $q$. There are many possible suspensions of an IET but there is a natural class of suspensions which gives the simplest possible surfaces. Namely the following was shown in [V1, §6], [Ma, §3] (see also the discussion in [KoZo, §1.2]):

Proposition 2.1. There is a unique stratum $\mathcal{H}$ of translation surfaces containing suspensions of $\mathcal{I} \mathcal{E}$ so that if $(q, \gamma)$ is a suspension then $q \in \mathcal{H}$ if and only if every vertical separatrix on $q$ intersects $\gamma(\mathbb{T})$. Every such suspension lies in the same connected component of $\mathcal{H}$.

Given $\mathcal{I E}$, we will call this stratum the stratum of minimal complexity corresponding to $\mathcal{I E}$. Recall that our strata have labeled singularities. It will be convenient to specify a labeling of singular points of a suspension, which depends on $\mathcal{I E}$ but is independent of the suspension. To this end note that if $(q, \gamma)$ is a suspension of $\mathcal{I} \mathcal{E}$, then the vertical straight-line flow of the image under $\gamma$ of a discontinuity of $\mathcal{I} \mathcal{E}$ must hit a singularity. Now suppose $q$ is in the minimal complexity stratum $\mathcal{H}$ of Proposition 2.1. Then a singularity of order $r$ is associated to $r+1$ discontinuities of $\mathcal{I} \mathcal{E}$. The choice of $q$ thus determines a partition $P$ of the discontinuities of $\mathcal{I} \mathcal{E}$. Conversely, it was shown by Veech that the partition $P$ can be recovered from the combinatorics of $\mathcal{I E}$ (as orbits of the auxiliary permutation $\sigma$ of $[\mathrm{V1}, \S 2]$ ) and is independent of the choice of the suspension. So if $(q, \gamma)$ is a suspension with $q \in \mathcal{H}$ then there is a bijection between the singularities of $q$ and elements of the partition $P$, where the number of partition elements is the number of singularities and a singularity of cone angle $2 \pi(k+1)$ corresponds to a block of size $k+1$ in $P$. We label the singularities of surfaces in $\mathcal{H}$ by elements of $P$, and given $\mathcal{I E}$, we will say that the suspension $(q, \gamma)$ is compatibly labeled if for any partition element $[d] \in P$ and any discontinuity $d^{\prime} \in[d]$, the vertical straight-line flow applied to $\gamma\left(d^{\prime}\right)$ hits the singularity labeled by $[d]$. 
Fix $\mathcal{I E}$ and determine $\mathcal{H}$ using the Proposition above. Let $\left(q_{1}, \gamma_{1}\right)$ and $\left(q_{2}, \gamma_{2}\right)$ be two compatibly labeled suspensions of $\mathcal{I E}$ with $q_{1}, q_{2} \in$ $\mathcal{H}$. Corresponding to $\left(q_{1}, \gamma_{1}\right)$ and $\left(q_{2}, \gamma_{2}\right)$, there is a canonical isotopy class of homeomorphisms $\varphi: q_{1} \rightarrow q_{2}$. Homeomorphisms in this class preserve the labeling of singularities and the isotopies are taken to fix the singularities. This class contains a homeomorphism $\varphi: q_{1} \rightarrow q_{2}$ such that

(1) $\gamma_{2}(t)=\varphi \circ \gamma_{1}(t)$ for all $t \in \mathbb{T}$.

(2) $\varphi$ maps vertical leaves to vertical leaves, preserving their orientation.

This information does not completely specify $\varphi$, but any two such maps differ by precomposition by a homeomorphism of $q_{1}$ which preserves the vertical foliation and preserves $\gamma_{1}(\mathbb{T})$ pointwise. All such maps are isotopic, so the isotopy class $[\varphi]$ is well-defined and depends only on the suspensions.

2.3. Rel, Real-rel, Imaginary-rel. We describe the rel foliation as a foliation on $\mathcal{H}_{\mathrm{m}}(\mathbf{r})$ which descends to a well-defined foliation on $\mathcal{H}(\mathbf{r})$. We view our cohomology classes as equivalence classes of linear maps from the associated homology spaces. Observe there is a restriction map

$$
\text { Res : } H^{1}\left(S, \Sigma ; \mathbb{R}^{2}\right) \rightarrow H^{1}\left(S ; \mathbb{R}^{2}\right)
$$

which is obtained by mapping a cochain $H_{1}(S, \Sigma ; \mathbb{R}) \rightarrow \mathbb{R}^{2}$ to its restriction to the 'absolute periods' $H_{1}(S ; \mathbb{R}) \subset H_{1}(S, \Sigma ; \mathbb{R})$. This restriction map is part of the exact sequence in cohomology,

$$
H^{0}(S) \rightarrow H^{0}(\Sigma) \rightarrow H^{1}(S, \Sigma) \stackrel{\text { Res }}{\rightarrow} H^{1}(S) \rightarrow\{0\}
$$

(coefficients in $\mathbb{R}^{2}$ ), and we obtain a natural subspace

$$
\Re=\text { ker Res } \subset H^{1}\left(S, \Sigma ; \mathbb{R}^{2}\right),
$$

consisting of the cohomology classes which vanish on $H_{1}(S) \subset H_{1}(S, \Sigma)$. Since the sequence (2.6) is invariant under homeomorphisms in $\operatorname{Mod}(S, \Sigma)$, the subspace $\mathfrak{R}$ is $\operatorname{Mod}(S, \Sigma)$-invariant. Since hol is equivariant with respect to the action of the group $\operatorname{Mod}(S, \Sigma)$ on $\mathcal{H}_{\mathrm{m}}(\mathbf{r})$ and $H^{1}\left(S, \Sigma ; \mathbb{R}^{2}\right)$, the foliation of $H^{1}\left(S, \Sigma ; \mathbb{R}^{2}\right)$ by cosets of the subspace $\mathfrak{R}$ induces by pullback a foliation of $\mathcal{H}_{\mathrm{m}}(\mathbf{r})$, and descends to a well-defined foliation on $\mathcal{H}(\mathbf{r})=\mathcal{H}_{\mathrm{m}}(\mathbf{r}) / \operatorname{Mod}(S, \Sigma)$. The area of a translation surface can be computed using the cup product pairing in absolute cohomology and hence the foliation preserves the area of surfaces, and in particular we obtain a foliation of a fixed area sublocus $\mathcal{H}$ (see [BSW] for more details). This foliation is called the rel foliation. Two nearby translation surfaces $q$ and $q^{\prime}$ are in the same plaque if the integrals of 
the flat structures along all closed curves are the same on $q$ and $q^{\prime}$. Intuitively, $q^{\prime}$ is obtained from $q$ by fixing one singularity as a reference point and moving the other singularity. Recall our convention that singularities are labeled, that is $\operatorname{Mod}(S, \Sigma)$ does not permute the singular points. Using this one can show that $\operatorname{Mod}(S, \Sigma)$ acts trivially on $\mathfrak{R} \cong H^{0}(\Sigma ; \mathbb{R}) / H^{0}(S, \mathbb{R})$ and hence the leaves of the rel foliation are equipped with a natural translation structure, modeled on $\mathfrak{R}$. The leaves of the rel foliation have (real) dimension $2(k-1)$ (where $k=|\Sigma|$ ). In this paper we will focus on the case $k=2$, so that rel leaves are 2-dimensional. We can integrate a cocycle $c \in \mathfrak{R}$ on any path joining distinct singularities and the resulting vector in $\mathbb{R}^{2}$ will be independent of the path, since any two paths differ by an element of $H_{1}(S)$. Thus in the case $k=2$ we obtain an identification of $\mathfrak{R}$ with $\mathbb{R}^{2}$ by the map $u \mapsto u(\delta)$ for any path joining the singularities. Our convention for this identification will be that we take a path $\delta$ oriented from $\xi_{1}$ to $\xi_{2}$.

The existence of a translation structure on rel leaves implies that any vector $u \in \mathfrak{R}$ determines an everywhere-defined vector field on $\mathcal{H}$. We can apply standard facts about ordinary differential equations to integrate this vector field. This gives rise to paths $\psi(t)=\psi_{q, u}(t)$ such that $\psi(0)=q$ and $\frac{d}{d t} \psi(t) \equiv u$. We will denote the maximal domain of definition of $\psi_{q, u}$ by $I_{q, u}$. When $1 \in I_{q, u}$ we will say that $\operatorname{Rel}^{u} q$ is defined and write $\psi_{q, u}(1)=\operatorname{Rel}^{u} q$. Also, in the case $k=2$ we will write

$$
\operatorname{Rel}_{r}^{(h)} q=\operatorname{Rel}^{u} q \text { when } u=(r, 0),
$$

and

$$
\operatorname{Rel}_{s}^{(v)} q=\operatorname{Rel}^{u} q \text { when } u=(0, s) .
$$

These trajectories are called respectively the real-rel and imaginary-rel trajectories. We will use identical notations for $\mathbf{q} \in \mathcal{H}_{\mathrm{m}}(\mathbf{r})$, noting that since $\pi: \mathcal{H}_{\mathrm{m}}(\mathbf{r}) \rightarrow \mathcal{H}(\mathbf{r})$ is an orbifold covering map, $I_{\mathbf{q}, u}=I_{q, u}$ and $\pi\left(\operatorname{Rel}^{u} \mathbf{q}\right)=\operatorname{Rel}^{u} q$.

Note that the trajectories need not be defined for all time, i.e. $I_{q, u}$ need not coincide with $\mathbb{R}$. For instance this will happen when a saddle connection on $q$ is made to have length zero, i.e. if 'singularities collide'. It was shown in $\mathrm{MW}$ that this is the only obstruction to completeness of leaves. Namely, in the case $k=2$, the following holds:

Proposition 2.2. Let $\mathcal{H}$ be a stratum with two singular points, let $\mathbf{q} \in \mathcal{H}_{\mathrm{m}}$, and let $u \in \mathfrak{R}$. Then the following are equivalent:

- $\operatorname{Rel}^{u} \mathbf{q}$ is defined.

- For all saddle connections $\delta$ on $\mathbf{q}$, and all $s \in[0,1]$,

$$
\operatorname{hol}(\mathbf{q}, \delta)+s \cdot u(\delta) \neq 0 .
$$


Corollary 2.3. If $q$ has two singular points and no horizontal (resp. vertical) saddle connections joining distinct singularities, then $\operatorname{Rel}_{r}^{(h)} q$ (resp. $\left.\operatorname{Rel}_{s}^{(v)} q\right)$ is defined for all $r, s \in \mathbb{R}$.

From standard results about ordinary differential equations we have that the map $(q, u) \mapsto \operatorname{Rel}^{u} q$ is continuous on its domain of definition, and

$$
\operatorname{Rel}_{r_{1}}^{(h)}\left(\operatorname{Rel}_{r_{2}}^{(h)} q\right)=\operatorname{Rel}_{r_{1}+r_{2}}^{(h)}(q), \quad \operatorname{Rel}_{s_{1}}^{(v)}\left(\operatorname{Rel}_{s_{2}}^{(v)} q\right)=\operatorname{Rel}_{s_{1}+s_{2}}^{(v)}(q)
$$

(where defined). On the other hand we caution the reader that the rel plane field need not integrate as a group action, i.e. it is easy to find examples for which

$$
\operatorname{Rel}_{r}^{(h)}\left(\operatorname{Rel}_{s}^{(v)} q\right) \neq \operatorname{Rel}_{s}^{(v)}\left(\operatorname{Rel}_{r}^{(h)} q\right) .
$$

We let $G$ act on the stratum $\mathcal{H}$ in the usual way and also let $G$ act on $\mathbb{R}^{2}$ by its standard linear action. The action of $G$ is equivariant for the map hol used to define the translation structure on rel leaves, and so this induces an action of $G$ on the subspace $\mathfrak{R}$, and this leads to the following result (see [BSW] for more details):

Proposition 2.4. Let $x \in \mathcal{H}$ and let $u \in \mathfrak{R}$. If $\operatorname{Rel}^{u}(x)$ is defined and $g \in G$ then $\operatorname{Rel}^{g u}(g x)$ is defined and $g\left(\operatorname{Rel}^{u}(x)\right)=\operatorname{Rel}^{g u}(g x)$. In particular, if $q$ has two singular points and has no horizontal saddle connections joining distinct singularities, then for all $r, s, t \in \mathbb{R}$,

$$
g_{t} \operatorname{Rel}_{r}^{(h)} q=\operatorname{Rel}_{e^{t} r}^{(h)} g_{t} q \text { and } g_{t} \operatorname{Rel}_{s}^{(v)} q=\operatorname{Rel}_{e^{-t} s}^{(v)} g_{t} q .
$$

2.4. Rel and suspensions. For any $\mathbf{q}$ in any stratum $\mathcal{H}_{\mathrm{m}}$ of marked surfaces, one can pull back the foliation of $\mathbb{R}^{2}$ by vertical lines, via planar charts, to obtain a topological singular foliation $\mathcal{G}(\mathbf{q})$ on the surface $S$. Now suppose surfaces in $\mathcal{H}_{\mathrm{m}}$ have two singular points, and $\mathbf{q}^{\prime}=\operatorname{Rel}_{s}^{(v)} \mathbf{q}$ for some $s \in \mathbb{R}$. Then the pulled back foliation $\mathcal{G}\left(\mathbf{q}^{\prime}\right)$ is well-defined and topologically isomorphic to $\mathcal{G}$. That is, there is a homeomorphism $\rho: \mathbf{q} \rightarrow \mathbf{q}^{\prime}$, which maps each point to another point in its vertical leaf, and preserves the measure transverse to vertical leaves. The homeomorphism $\rho$ depends on certain choices but different choices give rise to homeomorphisms which are isotopic to $\rho$ via an isotopy moving points along vertical leaves. See [MW, Theorems 1.2 and 12.2].

Since $\rho$ only moves points inside their vertical leaf, and the definition of a suspension involves only motion inside vertical leaves, the following is an immediate consequence. 
Proposition 2.5. Let $q^{\prime}=\operatorname{Rel}_{s}^{(v)} q$ and $\rho: q \rightarrow q^{\prime}$ be as above. If $(q, \gamma)$ is a suspension of the $\mathcal{I E}: \mathbb{T} \rightarrow \mathbb{T}$ in the sense of $\$ 2.2$ then $\left(q^{\prime}, \rho \circ \gamma\right)$ is also a suspension of $\mathcal{I} \mathcal{E}$.

\section{The Arnoux-Yoccoz IET, Suspensions, and Rel Rays}

Arnoux and Yoccoz defined the surface $x_{0}$ by first introducing an interval exchange transformation of which this surface is a suspension. We will employ a similar strategy; in fact all surfaces in the imaginaryrel ray we are interested in will be suspensions of this IET.

3.1. Renormalization of the IET. Fix an integer $\mathbf{g} \geqslant 2$. Let $\alpha$ be the unique positive real solution to the polynomial equation

$$
\alpha+\alpha^{2}+\cdots+\alpha^{\mathbf{g}}=1 .
$$

It is known by work of [AY] that the multiplicative inverse $\alpha^{-1}$ of $\alpha$, is a Pisot number (i.e., the algebraic conjugates of $\alpha$ all lie in the interior of the unit circle) and that for $\mathbf{g} \geqslant 3$ the field $\mathbb{Q}(\alpha)$ is of degree $\mathbf{g}$ and is not totally real. The value of $\alpha$ is decreasing as $\mathbf{g} \rightarrow \infty$, dropping from the reciprocal of the golden mean (when $\mathbf{g}=2$ ) to $\frac{1}{2}$ in the limit as $\mathbf{g} \rightarrow \infty$.

Associated to $\mathrm{g}$ is an IET $\mathcal{I E}: \mathbb{T} \rightarrow \mathbb{T}$. When specifying coordinates we identify $\mathbb{T}$ with the half-open interval $[0,1)$. The interval exchange $\mathcal{I E}$ is a composition of two involutions, $\mathcal{I} \mathcal{E}=I_{2} \circ I_{1}$. We define $I_{1}$ : $[0,1) \rightarrow[0,1)$ by partitioning $[0,1)$ into $\mathrm{g}$ subintervals:

$$
J_{1}=[0, \alpha), J_{2}=\left[\alpha, \alpha+\alpha^{2}\right), \ldots, J_{\mathbf{g}}=\left[1-\alpha^{\mathbf{g}}, 1\right),
$$

where interval $J_{k}$ has length $\alpha^{k}$. Each interval $J_{k}$ is invariant under the map $I_{1}$, and the action of $I_{1}$ on $J_{k}$ satisfies

$$
\left.I_{1}\right|_{J_{k}}: J_{k} \rightarrow J_{k} ; \quad t \mapsto t+\frac{\alpha^{k}}{2} \quad\left(\bmod \alpha^{k}\right)
$$

That is, $I_{1}$ treats each $J_{k}$ as a circle $\mathbb{R} / \alpha^{k} \mathbb{Z}$ and rotates this circle halfway around. The second involution does the same to the full interval $[0,1)$;

$$
I_{2}:[0,1) \rightarrow[0,1) ; \quad t \mapsto t+\frac{1}{2} \quad(\bmod 1) .
$$

For us, the most important aspect of the IET $\mathcal{I E}$ is that it is renormalizable in the strongest sense: there is a return map to a subinterval which is essentially the same as the original map (up to uniform scaling and rotation). 
Theorem 3.1 (Arnoux-Yoccoz $[\mathrm{AY}]$ ). Let $\widehat{\mathcal{I E}}: \mathbb{R} / \alpha \mathbb{Z} \rightarrow \mathbb{R} / \alpha \mathbb{Z}$ be the first return map of $\mathcal{I E}$ to $[0, \alpha)$, with $[0, \alpha)$ viewed as a fundamental domain for $\mathbb{R} / \alpha \mathbb{Z}$. Define

$$
\psi: \mathbb{R} / \alpha \mathbb{Z} \rightarrow \mathbb{T} ; \quad s \quad \bmod \alpha \mathbb{Z} \mapsto \alpha^{-1} s+\frac{\alpha^{-1}-1}{2} \bmod \mathbb{Z} .
$$

(This map scales $\mathbb{R} / \alpha \mathbb{Z}$ to become $\mathbb{R} / \mathbb{Z}$ and then rotates by $\frac{\alpha^{-1}-1}{2}$.) Then $\psi \circ \widehat{\mathcal{I E}}=\mathcal{I E} \circ \psi$.

Arnoux and Yoccoz stated this for $\mathbf{g} \geqslant 3$ and Bowman observed that it also holds for $\mathbf{g}=2$ (see [AY, $\S 2$ ] and [Bow1, Appendix]). We give the proof for completeness below. Before doing this, we will prove the following lemma which contains information on the interplay between $\mathcal{I E}$ and the map $\psi$ defined above. This lemma will also be used for later arguments involving rel rays.

Lemma 3.2. Let $s \in[0, \alpha)$. Viewing $\mathcal{I E}$ as a map $[0,1) \rightarrow[0,1)$, we have:

(1) If $\mathcal{I E}(s) \geqslant \alpha$ then $\psi(s)=\alpha^{-1} \mathcal{I} \mathcal{E}(s)-1$.

(2) If $\mathcal{I E}(s) \leqslant \alpha$ then $\psi(s) \in J_{\mathrm{g}}$ and $\mathcal{I} \mathcal{E} \circ \psi(s)=\psi \circ \mathcal{I} \mathcal{E}(s)$.

Proof. Under the assumption that $s \in[0, \alpha)$, by definition of $\mathcal{I E}$, we have

$$
\mathcal{I E}(s)= \begin{cases}s+\frac{\alpha+1}{2} & \text { if } s<\frac{1-\alpha}{2} \\ s-\frac{1-\alpha}{2} & \text { if } \frac{1-\alpha}{2} \leqslant s<\frac{\alpha}{2}, \\ s+\frac{1-\alpha}{2} & \text { if } \frac{\alpha}{2} \leqslant s\end{cases}
$$

These values all lie in $[0,1)$.

Statement (1) involves the first and third cases of (3.3). Here

$$
\alpha^{-1} \mathcal{I} \mathcal{E}(s)-1= \begin{cases}\alpha^{-1} s+\frac{-1+\alpha^{-1}}{2} & \text { if } s<\frac{1-\alpha}{2}, \\ \alpha^{-1} s+\frac{\alpha^{-1}-3}{2} & \text { if } \frac{\alpha}{2} \leqslant s .\end{cases}
$$

In either case we have $\alpha^{-1} \mathcal{I E}(s)-1=\alpha^{-1} s+\frac{\alpha^{-1}-1}{2}(\bmod 1)$, which is the definition of $\psi$.

To see (2), we observe by computing with the second case of (3.3) that $\psi \circ \mathcal{I E}(s)=\alpha^{-1} s$. Also since $s \in\left[\frac{1-\alpha}{2}, \frac{\alpha}{2}\right)$, we can compute

$$
\psi(s)=\alpha^{-1} s+\frac{\alpha^{-1}-1}{2} \in\left[\alpha^{-1}-1, \frac{\alpha^{-1}}{2}\right) .
$$

Using the identity $\alpha^{-1}=2-\alpha^{\mathbf{g}}$, we see that $\psi(s) \in\left[1-\alpha^{\mathbf{g}}, 1-\frac{\alpha^{\mathbf{g}}}{2}\right)$, which is the left half of the interval $J_{\mathrm{g}}$. Using the same identity,

$$
\mathcal{I E} \circ \psi(s)=\psi(s)+\frac{\alpha^{\mathbf{g}}}{2}-\frac{1}{2}=\alpha^{-1} s .
$$


Proof of Theorem 3.1. Let $s \in[0, \alpha)$. We consider two cases. First suppose that $\mathcal{I E}(s)<\alpha$. Then by definition of the first return map, we have $\widehat{\mathcal{I E}}(s)=\mathcal{I E}(s)$. So, the conclusion that $\psi \circ \widehat{\mathcal{I E}}(s)=\mathcal{I E} \circ \psi(s)$ follows from statement (2) of Lemma 3.2 .

Now suppose that $\mathcal{I E}(s) \geqslant \alpha$. Observe that $\widehat{\mathcal{I E}}(s)=\mathcal{I E}^{2}(s)$. Using statement (1) of Lemma 3.2, we have

$$
\mathcal{I E} \circ \psi(s)=\mathcal{I E}\left(\alpha^{-1} \mathcal{I E}(s)-1\right) .
$$

Observe that the map $\zeta: x \mapsto \alpha^{-1} x-1$ sends the interval $J_{k}$ affinely onto $J_{k-1}$ for $k \in\{2, \ldots, \mathbf{g}\}$. Therefore, we observe that it commutes with the first involution $I_{1}$. So,

$$
\mathcal{I E} \circ \psi(s)=I_{2} \circ \zeta \circ I_{1} \circ \mathcal{I E}(s) .
$$

If we can show that $I_{2} \circ \zeta(x)=\psi \circ I_{2}(x)$ for $x=I_{1} \circ \mathcal{I E}(s)$, then we will complete the proof that $\psi \circ \widehat{\mathcal{I E}}(s)=\mathcal{I E} \circ \psi(s)$. One side is

$$
I_{2} \circ \zeta(x)=I_{2}\left(\alpha^{-1} x-1\right)=\alpha^{-1} x+\frac{1}{2}+\mathbb{Z} .
$$

Now note that $\mathcal{I E}(s) \geqslant \alpha$, so $x=I_{1} \circ \mathcal{I E}(s) \geqslant \alpha$ and therefore:

$$
\psi \circ I_{2}(x)=\alpha^{-1}\left(x-\frac{1}{2}\right)+\frac{\alpha^{-1}-1}{2} \quad \bmod \mathbb{Z}=\alpha^{-1} x-\frac{1}{2} \quad \bmod \mathbb{Z} .
$$

We have verified $I_{2} \circ \zeta(x)$ and $\psi \circ I_{2}(x)$ are equal in $\mathbb{T}$ as required.

3.2. Suspensions. Fix $\mathbf{g} \geqslant 2$. Consider the Arnoux-Yoccoz IET $\mathcal{I E}$ : $\mathbb{T} \rightarrow \mathbb{T}$. We work out the associated stratum of minimal complexity, and its connected component containing suspensions of $\mathcal{I E}$. We recall from $\mathrm{KoZ}$ ] that the stratum $\mathcal{H}(1,1)$ is connected, and that $\mathcal{H}(\mathbf{g}-$ $1, \mathbf{g}-1)$ has three connected components $\mathcal{H}^{\text {hyp }}(\mathbf{g}-1, \mathbf{g}-1), \mathcal{H}^{\text {odd }}(\mathbf{g}-$ $1, \mathbf{g}-1), \mathcal{H}^{\text {even }}(\mathbf{g}-1, \mathbf{g}-1)$ when $\mathbf{g}$ is odd and two components $\mathcal{H}^{\text {hyp }}(\mathbf{g}-$ $1, \mathbf{g}-1), \mathcal{H}^{\text {non-hyp }}(\mathbf{g}-1, \mathbf{g}-1)$ when $\mathbf{g} \geqslant 4$ is even.

Proposition 3.3. The stratum of minimal complexity for $\mathcal{I E}$ is $\mathcal{H}(\mathrm{g}-$ $1, \mathbf{g}-1)$. If $\mathbf{g} \geqslant 3$ is odd, then the connected component is $\mathcal{H}^{\text {odd }}(\mathbf{g}-$ $1, \mathbf{g}-1)$, and if $\mathbf{g} \geqslant 4$ is even then it is $\mathcal{H}^{\text {non-hyp }}(\mathbf{g}-1, \mathbf{g}-1)$.

Proof. We will exhibit an explicit suspension $\left(q_{\star}, \gamma_{\star}\right)$ of $\mathcal{I E}$ with the property that every vertical leaf of $q_{\star}$ intersects $\gamma_{\star}$. By Proposition 2.1 (following Veech), $q_{\star}$ must then lie in the stratum of minimal complexity. We will then determine the connected component of the corresponding stratum. 

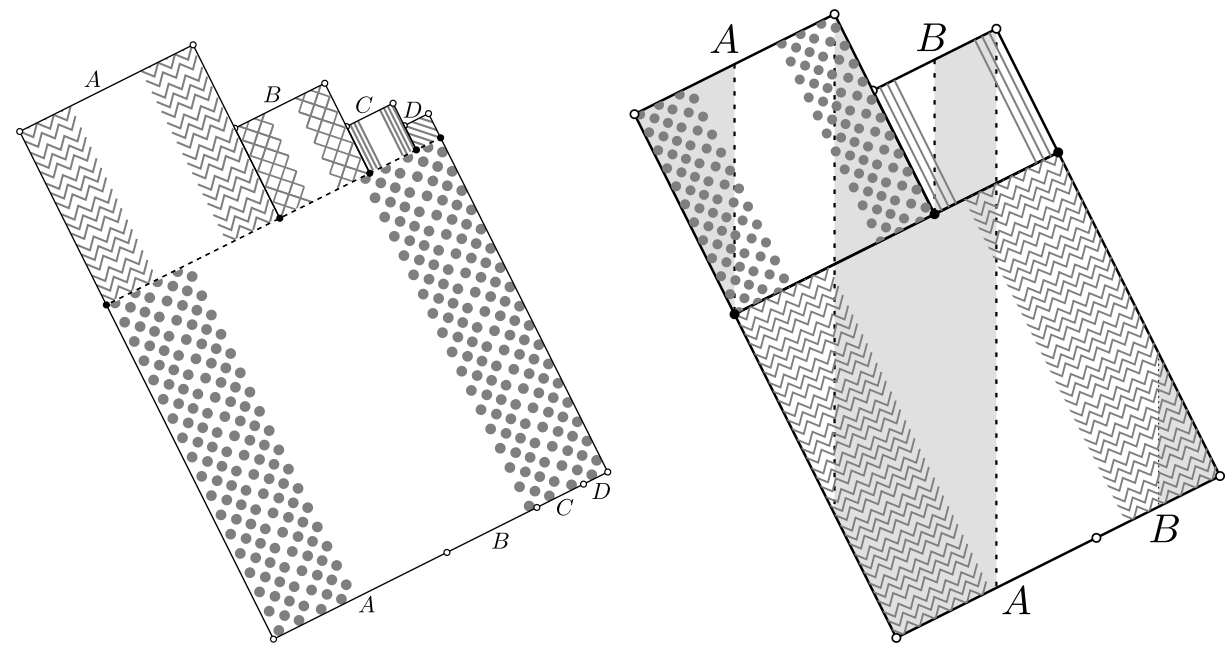

Figure 3 . The surface $q_{\star}$ from the proof of Proposition 3.3 with $\mathbf{g}=4$ (left) and $\mathbf{g}=2$ (right). Side identifications for edges of slope -2 are indicated by the hatching.

The suspension $q_{\star}$ will be made of $\mathbf{g}+1$ squares of dimensions $1 \times 1$, $\alpha \times \alpha, \ldots, \alpha^{\mathbf{g}} \times \alpha^{\mathbf{g}}$. See Figure 3 for examples. These squares are tilted so that the edges have slope $\frac{1}{2}$ and -2 . On each square, opposite sides of slope -2 are identified, to form a cylinder. The choice of slopes guarantees that a half rotation is performed when following the vertical straight-line flow from the bottom to the top of each of these cylinders, where the rotation is measured relative to the vertices of the square. The slope $\frac{1}{2}$ edges of the smaller squares are glued to the slope $\frac{1}{2}$ edge of the largest square. As we move from right to left over either slope $\frac{1}{2}$ edge of the largest square we glue to the other squares in order of largest to smallest. This defines $q_{\star}$.

The gluing rules imply that all the bottom corners of the small squares are identified with each other and all the top corners of the small squares are identified with each other. Thus there are two cone angles each of cone angle $2 \pi \mathbf{g}$; one lies on the top of the large square, and the other lies on the bottom. Therefore the surface $q_{\star}$ lies in the stratum $\mathcal{H}(\mathbf{g}-1, \mathbf{g}-1)$.

Let $\gamma_{\star}: \mathbb{T} \rightarrow q_{\star}$ be a constant speed parametrization of a geodesic core curve of the cylinder formed by the largest square, which we parameterize in such a way that $\gamma_{\star}(0)$ hits the top corner of this largest square under vertical straight-line flow. Based on the definition of $\mathcal{I} \mathcal{E}$ as a product of half rotations, we see that $\gamma_{\star}$ conjugates $\mathcal{I} \mathcal{E}$ to the return map of the vertical straight-line flow to $\gamma_{\star}(\mathbb{T})$. That is, $\left(q_{\star}, \gamma_{\star}\right)$ is a suspension of $\mathcal{I E}$. It also can be explicitly checked that every vertical 
separatrix intersects $\gamma_{\star}$, so $q_{\star}$ lies in the connected component of the minimal complexity stratum containing suspensions of $\mathcal{I} \mathcal{E}$.

The connected component of the stratum can we worked out following $\mathrm{KoZ}$. When $\mathrm{g} \geqslant 3$, the surface $q_{\star}$ is not hyperelliptic. (If $q_{\star}$ admitted an affine automorphism with derivative - Id, it would have to preserve all but the largest square, because the smaller squares are part of the Delaunay decomposition of $q_{\star}$. But because of the way these squares are glued to the largest square, rotating each of these squares halfway around does not extend to an automorphism of the full surface.) Using the classification of connected components KoZo, Theorem 1], we see that this determines the connected component containing these suspensions when $\mathbf{g}$ is even. When $\mathbf{g} \geqslant 3$ is odd, we need to consider the spin invariant. This can be computed using a symplectic basis for $H_{1}(S ; \mathbb{Z})$ where $S$ is the topological surface underlying the translation structure provided by $q_{\star}$. A symplectic basis is given by core curves of the $\mathbf{g}$ cylinders of slope -2 (each of which pass through one of the smaller squares and the largest square) together with core curves of the smallest $\mathrm{g}$ cylinders of slope $\frac{1}{2}$ (all but the one through the largest square). Since these are cylinders, the index of the field tangent to each curve is zero; see [KoZo, Lemma 2]. Thus the parity of the spin structure on $q_{\star}$ is given by $\mathbf{g}(\bmod 2)$; see KKoZo, equation (4)]. So when $\mathbf{g} \geqslant 3$ is odd, $q_{\star} \in \mathcal{H}^{\text {odd }}(\mathbf{g}-1, \mathbf{g}-1)$.

We will use $\bullet$ and $\circ$ to label the singularities of surfaces in $\mathcal{H}(\mathbf{g}-$ $1, \mathbf{g}-1)$. A suspension $(q, \gamma)$ with $q \in \mathcal{H}(\mathbf{g}-1, \mathbf{g}-1)$ is compatibly labeled if

(a) Whenever $t$ is an endpoint of $J_{k}$ from (3.2) for some $k$, the vertical straight-line flow of $\gamma(t)$ hits the singularity labeled $\bullet$.

(b) Whenever $t^{\prime}$ is a midpoint of an interval $J_{k}$, the vertical straightline flow applied to $\gamma\left(t^{\prime}\right)$ hits the singularity labeled $\circ$.

To see that this definition gives a well-defined labeling (i.e., that the label of a singularity is independent of the choice of $k$ ), we just need to check on one surface. The reader may consider the surfaces from the proof above. Statements (a) and (b) above define a partition of the discontinuities of $\mathcal{I E}$, and the reader can verify that the labeling is compatible in the sense of $\$ 2.2$, justifying our terminology.

The renormalizing map $\psi$ from Theorem 3.1 interacts with the suspensions of $\mathcal{I} \mathcal{E}$. Recall that $\psi: \mathbb{R} / \alpha \mathbb{Z} \rightarrow \mathbb{T}$ scales uniformly and rotates. Let $(q, \gamma)$ be a suspension of $\mathcal{I} \mathcal{E}$ with $q \in \mathcal{H}(\mathbf{g}-1, \mathbf{g}-1)$, and consider the map $\gamma \circ \psi^{-1}: \mathbb{T} \rightarrow q$ (where we treat $\psi^{-1}$ as a map $\mathbb{T} \rightarrow[0, \alpha)$ ). This map is not continuous, because $\gamma(0) \neq \gamma(\alpha)$. We will fix this by pushing $\gamma \circ \psi^{-1}$ along the leaves of the vertical foliation. Observe that 
both 0 and $\alpha$ are discontinuities for the map $\mathcal{I E}$, and

$$
\mathcal{I E}(0)=\lim _{t \rightarrow \alpha-} \mathcal{I E}(t)=\frac{1+\alpha}{2} .
$$

Let $F_{s}: q \rightarrow q$ be the vertical straight-line flow. From the above, we have

$$
\gamma\left(\frac{1+\alpha}{2}\right)=\lim _{t \rightarrow 0+} F_{r(t)} \gamma(t)=\lim _{t \rightarrow \alpha-} F_{r(t)} \gamma(t),
$$

where $r(t)$ denotes the return time of $\gamma(t)$ to $\gamma(\mathbb{T})$ under the flow $\left\{F_{t}\right\}$. Choose a continuous function $s:[0, \alpha] \rightarrow \mathbb{R}$ satisfying $s(0)=r(0)$, $s\left(\frac{\alpha}{2}\right)=0, s(\alpha)=r(\alpha)$ and $0 \leqslant s(t) \leqslant r(t)$ for all $t \in(0, \alpha)$. (The value $\frac{\alpha}{2}$ is singled out because it is the only discontinuity of $\mathcal{I E}$ in the open interval $(0, \alpha))$. These choices make the following a well-defined continuous curve:

$$
\hat{\gamma}: \mathbb{T} \rightarrow q ; \quad t \mapsto \begin{cases}\gamma\left(\frac{1+\alpha}{2}\right) & \text { if } \psi^{-1}(t)=0 \\ F_{s(t)} \circ \gamma \circ \psi^{-1}(t) & \text { otherwise. }\end{cases}
$$

This new curve is well-defined because when $\psi^{-1}(t) \neq 0$, the vertical straight-line flow of $\gamma \circ \psi^{-1}(t)$ does not hit a singularity up to time $s(t)$. Although $\hat{\gamma}$ depends on the choice of the function $s(t)$, different choices will only change $\hat{\gamma}$ by an isotopy along vertical leaves. As this will not affect our arguments we suppress the dependence on $s(t)$ from the notation. We denote by $\gamma \mapsto \hat{\gamma}$ this operation which produces a new curve $\hat{\gamma}$ from $(q, \gamma)$.

We have the following corollary to Theorem 3.1. The proof is a simple calculation which we leave to the reader.

Corollary 3.4. If $(q, \gamma)$ is a compatibly labeled suspension of $\mathcal{I E}$ with $q \in \mathcal{H}(\mathbf{g}-1, \mathbf{g}-1)$, then so is $(q, \hat{\gamma})$.

Definition 3.5. In the context of the corollary, let $\varphi: q \rightarrow q$ be a homeomorphism from the canonical isotopy class corresponding to the suspensions $(q, \gamma)$ and $(q, \hat{\gamma})$ (see [2.2). We call $[\varphi]$ the renormalization mapping class of the suspension $(q, \gamma)$.

3.3. Completely periodic rel rays. In this section, we will describe an imaginary-rel ray in $\mathcal{H}(\mathbf{g}-1, \mathbf{g}-1)$ so that every surface on the ray is horizontally completely periodic. In this section we fix $\mathbf{g} \geqslant 2$. The argument is the same for each $\mathbf{g}$.

We will describe a surface $q_{0} \in \mathcal{H}(\mathbf{g}-1, \mathbf{g}-1)$ which is horizontally completely periodic and is a suspension of $\mathcal{I} \mathcal{E}$. We present $q_{0}$ as a disjoint union of rectangles with edge identifications. We define the 


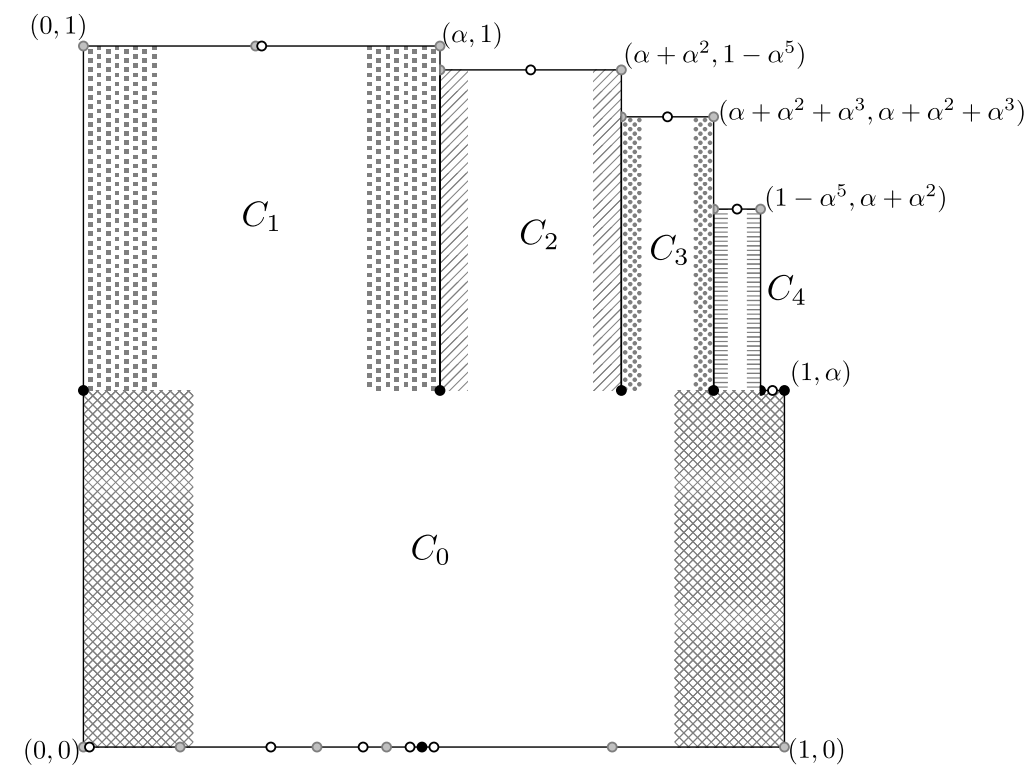

FiguRE 4 . The surface $q_{0}$ with genus $\mathbf{g}=5$, is made up of five horizontal cylinders. A point $(x, y)$ on a top boundary is glued to the point $(\mathcal{I E}(x), 0)$.

rectangles

$$
\begin{gathered}
R_{0}=[0,1] \times[0, \alpha] \\
R_{k}=\overline{J_{k}} \times\left[\alpha, \sum_{j=1}^{\mathbf{g}-k+1} \alpha^{j}\right] \quad \text { for } k \in\{1, \ldots, \mathbf{g}-1\}
\end{gathered}
$$

and with $\overline{J_{k}}$ the closure of the interval $J_{k}$ from $(3.2)$. The edge identifications follow:

- We identify opposite vertical sides of $R_{k}$ for $k \in\{0, \ldots, \mathbf{g}-1\}$ by a horizontal translation, so that each $R_{k}$ forms a horizontal cylinder $C_{k}$ on $q_{0}$.

- We glue the bottom edge of $R_{k}$ for $k \in\{1, \ldots, \mathrm{g}-1\}$ with the top edge of $C_{0}$ by the identity map (the bottom edge of each $R_{k}$ is a subset of the top edge of $R_{0}$, viewed as subsets of $\mathbb{R}^{2}$ ).

- If $(x, y)$ is in the top edge of $R_{k}$ for $k \in\{1, \ldots, \mathbf{g}-1\}$ or if $(x, y)$ is in the top edge of $C_{0}$ with $x \in J_{\mathbf{g}}$, then we identify $(x, y)$ with $(\mathcal{I E}(x), 0)$ in the bottom of $C_{0}$.

We label the singularities so that $q_{0}$ is a compatibly labeled suspension of $\mathcal{I E}$. Figure 4 shows an example of the surface $q_{0}$. 
Note that $q_{0}$ is naturally a measure preserving suspension of $\mathcal{I} \mathcal{E}$. To see this define $\gamma_{0}$ to be a core curve of $C_{0}$ via

$$
\gamma_{0}: \mathbb{T} \rightarrow q_{0} ; \quad x \mapsto\left(x, \frac{\alpha}{2}\right) \in C_{0}
$$

It should be clear that because of the identifications above, $\gamma_{0}$ conjugates $\mathcal{I E}$ to the first return map of the vertical straight-line flow to $\gamma_{0}(\mathbb{T})$. Recall that Corollary 3.4 described a second suspension $\left(q_{0}, \hat{\gamma}_{0}\right)$ constructible from $\left(q_{0}, \gamma_{0}\right)$. Reviewing the definitions, we see that:

Proposition 3.6. Up to isotopy along vertical leaves, the curve $\hat{\gamma}_{0}$ on $q_{0}$ is defined by

$$
\hat{\gamma}_{0}: \mathbb{T} \rightarrow q_{0} ; \quad x \mapsto\left(\psi^{-1}(x), \alpha+\frac{\alpha}{2}\right)
$$

which is a parameterized core curve of the cylinder $C_{1}$.

We will be investigating the imaginary rel leaf through $q_{0}$. Define $q_{s}=\operatorname{Rel}_{s}^{(v)} q_{0}$ whenever this definition makes sense. By Proposition 2.5. we can push $\gamma_{0}$ through the rel leaf obtaining parameterized curves $\gamma_{s}$ in $q_{s}$ taken from the same homotopy class (up to this deformation) which make $\left(q_{s}, \gamma_{s}\right)$ also a compatibly labeled measure preserving suspension of $\mathcal{I E}$.

The following is our main result on the imaginary rel leaf through $q_{0}$.

Theorem 3.7. Fix $\mathbf{g} \geqslant 2$, and let $q_{0}$ be the surface defined above. Define

$$
\beta=\frac{\alpha^{2}}{1-\alpha}=\sum_{j=-\infty}^{-2} \alpha^{-j}=\alpha^{2}+\alpha^{3}+\alpha^{4}+\cdots
$$

Then the surface $q_{s}=\operatorname{Rel}_{s}^{(v)} q_{0}$ is well-defined for every $s>-\beta$. Define $x_{t}=q_{t-\beta}$ for all $t>0$. Then:

(1) For every $t>0$, we have

$$
\widetilde{g} x_{\alpha^{-1} t}=x_{t}, \quad \text { where } \tilde{g}=\left(\begin{array}{rr}
\alpha^{-1} & 0 \\
0 & \alpha
\end{array}\right) \text {. }
$$

Thus there is an affine homeomorphism $\varphi_{t}: x_{\alpha^{-1} t} \rightarrow x_{t}$ with derivative $\widetilde{g}$. Let $\rho_{t}: x_{t} \rightarrow x_{\alpha^{-1} t}$ be a homeomorphism obtained by the rel deformation as in $\$ 2.4$. Then the composition $\varphi_{t} \circ \rho_{t}$ : $x_{t} \rightarrow x_{t}$ lies in the renormalization mapping class of $\left(x_{t}, \gamma_{t-\beta}\right)$. 


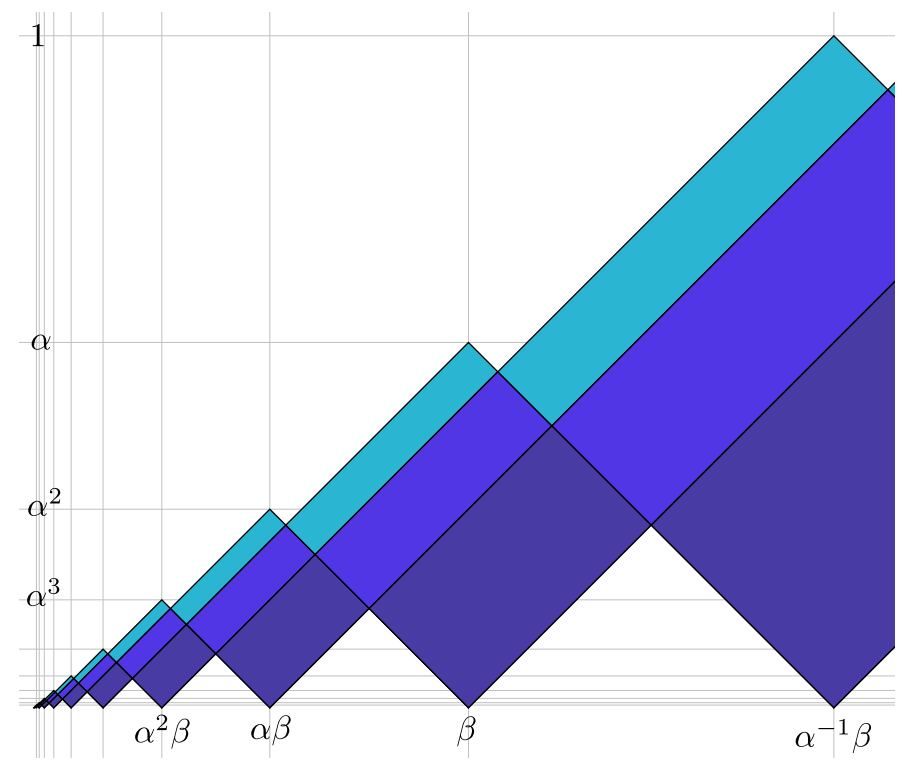

FiguRE 5. Black lines represent heights of cylinders in $x_{t}$ as a function of $t$ (with $\mathbf{g}=3$ ).

(2) Every surface $x_{t}$ with $t>0$ is horizontally completely periodic. Concretely, let $k$ be the integer so that

$$
\sum_{j=-\infty}^{k} \alpha^{-j} \leqslant t<\sum_{j=-\infty}^{k+1} \alpha^{-j} .
$$

There are two distinct combinatorial cases:

(a) If equality is attained on the left of (3.9) then $x_{t}$ has a horizontal cylinder decomposition with $\mathbf{g}$ cylinders with circumferences $\alpha^{k+2}, \alpha^{k+3}, \alpha^{k+4}, \ldots, \alpha^{k+\mathbf{g}+1}$.

(b) Otherwise, $x_{t}$ has a horizontal cylinder decomposition with $\mathbf{g}+1$ cylinders with circumferences $\alpha^{k+2}, \alpha^{k+3}, \ldots, \alpha^{k+\mathbf{g}+2}$. In this case, each cylinder has only one singularity in each of its boundary components, where the cylinder of greatest circumference has - appearing on its top component, and - appearing on its bottom component, and the other cylinders have $\circ$ on their top component and $\bullet$ on their bottom component.

The proof will show that the heights of the cylinders in $x_{t}$ can also be explicitly described, see Figure 5 .

The proof of Theorem 3.7 occupies the rest of this subsection. Consider the imaginary-rel flow applied to $q_{0}$ for distance $s$, by which we defined the surface $q_{s}$. This flow moves the white singularity $\circ$ upward, 


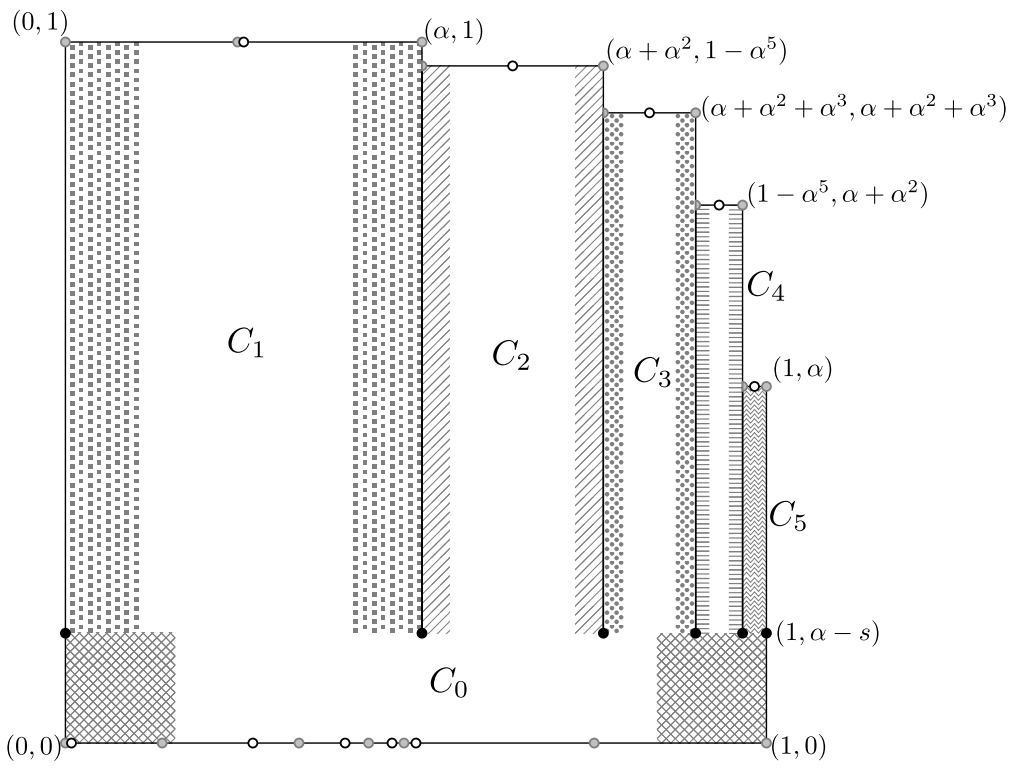

Figure 6 . The surface $q_{s}$ with $0<s<\alpha$ when $\mathbf{g}=5$. Again, a point $(x, y)$ on a top boundary is glued to the point $(\mathcal{I E}(x), 0)$.

or equivalently, moves the black singularity • downward. We will describe an explicit construction of $\operatorname{Rel}_{s}^{(v)} q_{0}$ (following [McM4]). Since • has cone angle $2 \pi \mathbf{g}$, there are $\mathbf{g}$ downward pointing prongs starting at this singularity, and we form downward pointing slits starting at these prongs of length $s$. This construction works as long as $\bullet$ is not the top point of a vertical saddle connection of length at most $s$. Define a new translation surface by using the same polygonal presentation, keeping all gluing maps the same, except for the gluing along the slits. Along the slits we reglue so that the former location of the black singularity is replaced by $\mathbf{g}$ regular points. This determines the gluings of the slits, and the bottom endpoints of the slits are forced to be identified into a $2 \pi \mathrm{g}$ cone singularity which we label $\bullet$ in the new surface. By examining the effect on holonomies of paths, it can be observed that the resulting surface is indeed $\operatorname{Rel}_{s}^{(v)} q_{0}$; that is for every $\gamma$ joining a singular point to itself, its holonomy is unchanged by this surgery, and for any directed $\gamma$ from the black singularity to the white singularity, its holonomy changes by adding $(0, s)$. We will refer to this presentation of the imaginary-rel surgery as the slit construction.

First consider the slit construction applied to construct $q_{s}=\operatorname{Rel}_{s}^{(v)} q_{0}$ for $0<s<\alpha$. The $\mathbf{g}$ downward slits cut into but not entirely through the cylinder $C_{0}$. Regluing the slits has the effect of shortening the 
cylinder $C_{0}$ by $s$ and heightening the cylinders $C_{1}, \ldots, C_{\mathbf{g}-1}$ by $s$. This surgery also creates a new cylinder which we call $C_{\mathbf{g}}$. This cylinder emerges from the interval $J_{\mathrm{g}} \times\{\alpha\}$, where the top boundary of $C_{0}$ touches the bottom boundary of $C_{0}$. The segment $J_{\mathbf{g}} \times\{\alpha\}$ has $\circ$ as its midpoint and $\bullet$ as its endpoints. The slits we cut move downward from these two endpoints. Since the other edges of the slits have been accounted for, we must be gluing the slits on either side of this interval together to make a new cylinder. A surface of this form is shown in Figure 6.

Proposition 3.8. For $0<s<\alpha$, the surface $q_{s}$ admits a horizontal cylinder decomposition with $\mathbf{g}+1$ cylinders $C_{0}, \ldots, C_{\mathbf{g}}$. The circumferences of cylinder $C_{k}$ is $\alpha^{k}$, while the height $h_{k}$ of the cylinder $C_{k}$ is described by $h_{0}=\alpha-s$ and

$$
h_{k}=s+\sum_{j=2}^{\mathbf{g}-k+1} \alpha^{j}=s+\beta-\alpha^{\mathbf{g}-k} \beta \quad \text { for } k=1, \ldots, \mathbf{g} .
$$

The homology classes $\left[C_{k}\right]$ of core curves of these cylinders oriented rightward are linearly independent as elements of $H_{1}(S \backslash \Sigma, \mathbb{Q})$.

Proof. All but the last sentence should be clear from the discussion above. Consider that the holonomy map hol $: H_{1}(S \backslash \Sigma, \mathbb{Q}) \rightarrow \mathbb{R}^{2}$ is $\mathbb{Q}$ linear. Observe $\operatorname{hol}_{x}\left(\left[C_{i}\right]\right)=\alpha^{i}$. Letting $W=\operatorname{span}_{\mathbb{Q}}\left\{\left[C_{0}\right], \ldots,\left[C_{\mathbf{g}-1}\right]\right\}$ (omitting $\left[C_{\mathbf{g}}\right]$ ) we see $\operatorname{hol}_{x}(W)=\mathbb{Q}(\alpha)$. Since $\alpha$ is an algebraic number of degree $\mathbf{g}$ [AY, Lemma 3], we know that hol $\left.\right|_{W}$ is injective and $\operatorname{dim}_{\mathbb{Q}}(W)=\mathrm{g}$. Using the description of $q_{s}$ provided above we can see that $\left[C_{0}\right]-\left[C_{1}\right]-\left[C_{2}\right]-\cdots-\left[C_{\mathbf{g}}\right]$ can be represented by a loop which wraps once around $\bullet$ in the counterclockwise direction. This nontrivial class is not in $W$ since hol $\left.\right|_{W}$ is injective, and hence $\left[C_{\mathbf{g}}\right] \notin W$. Thus the homology classes of these $\mathbf{g}+1$ cylinders are linearly independent over $\mathbb{Q}$.

Now consider using the slit construction to produce $q_{\alpha}$. We think of the slit construction as manipulating the presentation of $q_{0}$. Slits of length $\alpha$ cut entirely across the rectangle $R_{0}$, and after the identifications of slits each of the rectangles $R_{k}$ for $k \in\{1, \ldots, \mathbf{g}-1\}$ has been made taller by $\alpha$. Also, a new rectangle is formed below the interval $J_{\mathrm{g}} \times\{\alpha\}$. We see that the surface $q_{\alpha}$ can be presented as a disjoint union of rectangles with edge identifications. These rectangles are

$$
R_{k}^{\prime}=\overline{J_{k}} \times\left[0, \sum_{j=1}^{\mathbf{g}-k+1} \alpha^{j}\right] \quad \text { for } k \in\{1, \ldots, \mathbf{g}\} .
$$

Edge identifications are given by: 


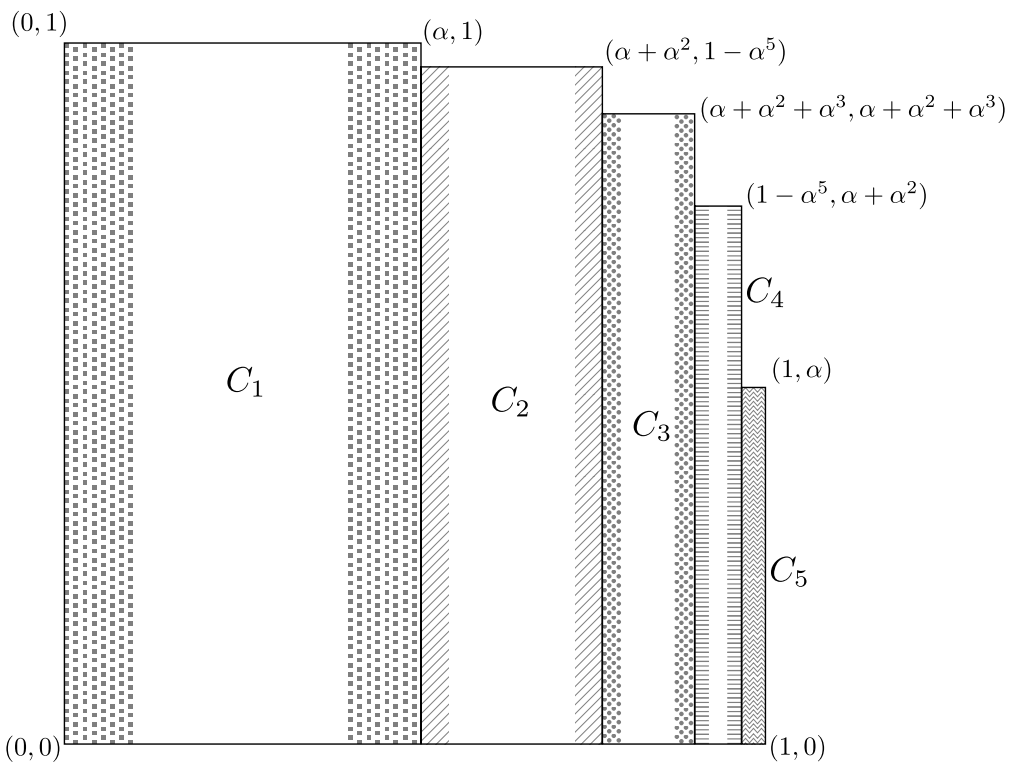

Figure 7 . The surface $q_{\alpha}$ when $\mathbf{g}=5$. Again, a point $(x, y)$ on a top boundary is glued to the point $(\mathcal{I E}(x), 0)$.

- Opposite vertical edges of each $R_{k}^{\prime}$ are glued by translation to form cylinders $C_{k}^{\prime}$ for $k \in\{1, \ldots, \mathbf{g}\}$.

- If $(x, y)$ is a point in the interior of a top edge of some $R_{k}$, we identify it to $(\mathcal{I E}(x), 0)$ in the bottom edge of one of the rectangles. We extend this identification continuously to the corners.

Figure 7 shows an example presentation.

The following is the main ingredient in the proof of Theorem 3.7 .

Lemma 3.9. There is an affine homeomorphism $\varphi: q_{\alpha} \rightarrow q_{0}$ with derivative $\tilde{g}$ (as in (3.8)), which carries each cylinder $C_{i+1}^{\prime}$ of $q_{\alpha}$ to cylinder $C_{i}$ of $q_{0}$. Let $\rho: q_{0} \rightarrow q_{\alpha}$ be a homeomorphism moving points in their vertical leaf, obtained from the deformation through the imaginary rel leaf (see \$2.4). Then $\varphi \circ \rho$ is a representative of the renormalization mapping class of the suspension $\left(q_{0}, \gamma_{0}\right)$.

Proof. We will explicitly define an affine homeomorphism $\varphi: q_{\alpha} \rightarrow q_{0}$ with derivative $\tilde{g}$. We begin by defining $\varphi$ on the cylinder $C_{1}^{\prime}$. Recall that we think of $C_{1}^{\prime}$ as $R_{1}^{\prime}$ with vertical edges identified by horizontal translation. Consider the map

$$
\varphi_{1}: R_{1}^{\prime} \rightarrow C_{0} ; \quad(x, y) \mapsto(\psi(x), \alpha y),
$$


where $\psi$ is the renormalizing map from Theorem 3.1. Observe that this map descends to a well-defined affine map $C_{1}^{\prime} \rightarrow C_{0}$ with derivative $\widetilde{g}$. For $k \in\{2, \ldots \mathrm{g}\}$, we define affine bijections

$$
\varphi_{k}: R_{k}^{\prime} \rightarrow R_{k-1} ; \quad(x, y) \mapsto \widetilde{g}(x, y)+(-1, \alpha) .
$$

The check that this is really an affine bijection is left to the reader. These maps descend to well-defined maps $C_{k}^{\prime} \rightarrow C_{k-1}$ for $k \geqslant 2$.

We define $\varphi: q_{\alpha} \rightarrow q_{0}$ by requiring that its restriction to each $C_{k}^{\prime}$ is $\varphi_{k}$, for $k \in\{1, \ldots, \mathbf{g}\}$. For this to be well-defined, we need to show that the maps $\varphi_{k}$ agree on the horizontal boundaries of the cylinders. We'll write $\left(x_{1}, y_{1}\right)_{j} \sim\left(x_{2}, y_{2}\right)_{k}$ to denote that $\left(x_{1}, y_{1}\right) \in R_{j}$ is identified with $\left(x_{2}, y_{2}\right) \in R_{k}$ along a horizontal edge in $q_{0}$. We'll use similar notation but with the equivalence relation $\approx$ to denote this equivalence for $q_{\alpha}$.

First consider a point $(x, 1)$ taken from $C_{1}^{\prime}$ such that $\mathcal{I E}(x) \geqslant \alpha$. Then we have

$$
(x, 1)_{1} \approx(\mathcal{I E}(x), 0)_{k} \text { for some } k \in\{2, \ldots, \mathbf{g}\} .
$$

We need to check that the images of these points are identified, i.e., that

$$
\varphi_{1}(x, 1)_{0} \sim \varphi_{k}(\mathcal{I E}(x), 0)_{k-1}
$$

We compute:

$$
\varphi_{1}(x, 1)=(\psi(x), \alpha) \text { and } \varphi_{k}(\mathcal{I E}(x), 0)=\left(\alpha^{-1} \mathcal{I} \mathcal{E}(x)-1, \alpha\right) .
$$

Equality of these values points is given by (1) of Lemma 3.2 so these points are identified $x_{\alpha}$.

Now suppose that $(x, 1)$ is taken from $C_{1}^{\prime}$ and $\mathcal{I E}(x)<\alpha$. Then,

$$
(x, 1)_{1} \approx(\mathcal{I E}(x), 0)_{1} .
$$

We need to check that

$$
\varphi_{1}(x, 1)_{0} \sim \varphi_{1}(\mathcal{I E}(x), 0)_{0} .
$$

We have $\varphi_{1}(x, 1)=(\psi(x), \alpha)$. By statement (2) of Lemma 3.2, we know that $\psi(x) \in J_{\mathrm{g}}$. So from our description of $x_{\alpha}$,

$$
(\psi(x), \alpha)_{1} \sim(\mathcal{I E} \circ \psi(x), 0)_{1} .
$$

On the other hand, we have

$$
\varphi_{1}(\mathcal{I E}(x), 0)=(\psi \circ \mathcal{I E}(x), 0)=(\mathcal{I E} \circ \psi(x), 0)
$$

by the remainder of statement (2). We have shown (3.11) as desired.

We have shown that our map is well defined on the top edge of $C_{1}^{\prime}$. Now consider a point $(x, y)$ in the top of $C_{k}^{\prime}$ for some $k \in\{2, \ldots, \mathbf{g}\}$. 
Observe that $\mathcal{I E}(x) \in J_{1}$ because $x \geqslant \alpha$. From our description of $x_{\alpha}$, we have

$$
(x, y)_{k} \approx(\mathcal{I E}(x), 0)_{1} .
$$

So, we need to check that

$$
\varphi_{k}(x, y)_{k-1} \sim \varphi_{1}(\mathcal{I E}(x), 0)_{0} .
$$

Let $x_{0}=\mathcal{I E}^{-1}(x)$, which is in the interval $J_{1}$ by definition of $\mathcal{I E}$. Then,

$$
\varphi_{k}(x, y)_{k-1}=\left(\alpha^{-1} \mathcal{I} \mathcal{E}\left(x_{0}\right)-1, \alpha(y+1)\right)=\left(\psi\left(x_{0}\right), \alpha(y+1)\right),
$$

where we use (2) of Lemma 3.2. The $y$-coordinate must lie on a top edge by prior work. Using the gluing rules in $q_{\alpha}$, we have

$$
\varphi_{k}(x, y)_{k-1} \sim\left(\mathcal{I E} \circ \psi\left(x_{0}\right), 0\right)_{0} .
$$

Letting $\widehat{\mathcal{I E}}$ be the return map to $J_{1}$ as in Theorem 3.1. Note that $\widehat{\mathcal{I E}}\left(x_{0}\right)=\mathcal{I E}^{2}\left(x_{0}\right)$, and using this theorem, we see

$$
\mathcal{I E} \circ \psi\left(x_{0}\right)=\psi \circ \widehat{\mathcal{I E}}\left(x_{0}\right)=\psi \circ \mathcal{I E}^{2}\left(x_{0}\right) .
$$

Since $\varphi_{1}(\mathcal{I E}(x), 0)=\left(\psi \circ \mathcal{I E}^{2}\left(x_{0}\right), 0\right)$, we have verified 3.12$)$.

Now we will discuss the remaining statements. We can realize $\rho$ as in the Lemma by a homeomorphism $\rho: q_{0} \rightarrow q_{\alpha}$ as stated in the Lemma which carries each cylinder $C_{k}$ to $C_{k}^{\prime}$ for $k=1, \ldots, \mathbf{g}-1$. By Proposition 3.6, the curve $\hat{\gamma}_{0}$ can be taken to be a core curve of $C_{1}$ with the provided parametrization (3.6). Then up to homotopy along the leaves, the image under $\rho$ of $\hat{\gamma}_{0}$ can be taken to be a core curve of $C_{1}^{\prime}$ with the $x$-coordinate parameterized as in (3.6). We observe that our definition of $\varphi$ carries $\rho \circ \hat{\gamma}_{0}$ to $\gamma_{0}$ up to homotopy along the leaves. Since the composition $\varphi \circ \rho$ also respects the vertical foliation, it is in the renormalization mapping class of the suspension $\left(q_{0}, \gamma_{0}\right)$.

Proof of Theorem 3.7. Define $\beta=\sum_{j=-\infty}^{-2} \alpha^{-j}$ and $x_{t}=q_{t-\beta}$ as in the statement of the theorem. By definition $x_{t}=\operatorname{Rel}_{t-\beta}^{(v)} x_{\beta}$.

We will begin by arguing that $x_{t}$ is well defined for $t>0$. Let $\widetilde{g}$ be as in the statement of the theorem. Through the use of $(2.8)$, we have

$$
\operatorname{Rel}_{\alpha^{-k} s}^{(v)} \circ \tilde{g}^{-k}(x)=\tilde{g}^{-k} \circ \operatorname{Rel}_{s}^{(v)}(x),
$$

for any $x \in \mathcal{H}$ and $k \in \mathbb{Z}$ for which at least one side of this equation is defined. Lemma 3.9 tells us that

$$
\operatorname{Rel}_{\alpha}^{(v)} x_{\beta}=\tilde{g}^{-1} x_{\beta}
$$

Define $r(k)=\sum_{j=-\infty}^{k-2} \alpha^{-j}$. We claim

$$
x_{r(k)}=\widetilde{g}^{-k} x_{\beta} \quad \text { for each } k \in \mathbb{Z} .
$$


Recalling that $\beta=\sum_{j=-\infty}^{-2} \alpha^{-j}=r(0)$, we see this is tautologically true when $k=0$ and true when $k=1$ by (3.14). We can verify this for all $k \in \mathbb{Z}$ by an induction using (3.13) which we leave to the reader. Observe that $r(k)=\alpha^{-k} \beta$, so we can rewrite (3.15) as

$$
x_{\alpha^{-k} \beta}=\widetilde{g}^{-k} x_{\beta} \text { for all } k \in \mathbb{Z} \text {. }
$$

Let $s \in[0, \alpha)$. Then, we have

$$
\begin{aligned}
x_{\alpha^{-k}(\beta+s)} & =\operatorname{Rel}_{\alpha^{-k} s}^{(v)} x_{\alpha^{-k} \beta}=\operatorname{Rel}_{\alpha^{-k} s}^{(v)} \widetilde{g}^{-k} x_{\beta} \\
& =\widetilde{g}^{-k} \operatorname{Rel}_{s}^{(v)} x_{\beta}=\widetilde{g}^{-k} x_{\beta+s}=\widetilde{g}^{-k} q_{s} .
\end{aligned}
$$

This shows that $x_{t}$ is defined for $t>0$.

We will now prove statement (1) of the theorem. Recall that Lemma 3.9 provided us with an affine homeomorphism $\varphi: x_{\alpha^{-1} \beta} \rightarrow x_{\beta}$ with derivative $\widetilde{g}$. Recall from $\$ 2.4$ that there is a natural isotopy class of homeomorphisms between surfaces on the same rel leaf, obtained by deforming along the leaf. Abusing notation we will choose a homeomorphism from this isotopy class and denote it by $\operatorname{Rel}_{s}^{(v)}$. For all $t>0$ define an isotopy class of homeomorphisms $\left[\varphi_{t}\right]$ from $x_{\alpha^{-1} t}$ to $x_{t}$ by the composition of maps

$$
x_{\alpha^{-1} t} \stackrel{\operatorname{Rel}_{\alpha^{-1}(\beta-t)}^{(v)}}{\longrightarrow} x_{\alpha^{-1} \beta} \stackrel{\varphi}{\rightarrow} x_{\beta} \stackrel{\operatorname{Rel}_{t-\beta}^{(v)}}{\longrightarrow} x_{t} .
$$

We will check that there is an affine homeomorphism $\varphi_{t}: x_{\alpha^{-1} t} \rightarrow x_{t}$ with derivative $\widetilde{g}$ in this isotopy class. It suffices to check that

$$
\operatorname{hol}\left(\varphi_{t}(\gamma), x_{t}\right)=\tilde{g} \operatorname{hol}\left(\gamma, x_{\alpha^{-1} t}\right) .
$$

for any relative cycle $\gamma \in H_{1}(S, \Sigma ; \mathbb{Z})$. Clearly this holds for an absolute cycle since the only thing in the composition which changes holonomy of an absolute cycle is the action of $\varphi$, which has derivative $\widetilde{g}$. Now consider a path $\gamma$ joining $\bullet$ to $\circ$ in $x_{\alpha^{-1} t}$. The action of $\operatorname{Rel}_{\alpha^{-1}(\beta-t)}^{(v)}$ changes its holonomy by adding $\left(0, \alpha^{-1}(\beta-t)\right)$, then the action of $\varphi$ scales by $\tilde{g}$, then the action of $\operatorname{Rel}_{t-\beta}^{(v)}$ changes holonomy by adding $(0, t-\beta)$. Thus

$$
\begin{aligned}
\operatorname{hol}\left(\varphi_{t}(\gamma), x_{t}\right) & =\widetilde{g}\left(\operatorname{hol}\left(\gamma, x_{t}\right)+\left(0, \alpha^{-1}(\beta-t)\right)\right)+(0, t-\beta) \\
& =\widetilde{g} \operatorname{hol}\left(\gamma, x_{t}\right)+(0, \beta-t)+(0, t-\beta)=\widetilde{g} \operatorname{hol}\left(\gamma, x_{t}\right) .
\end{aligned}
$$

This verifies (3.19), and implies the existence of an affine homeomorphism $\varphi_{t}$ in the class $\left[\varphi_{t}\right]$. Now consider the mapping class $\varphi_{t} \circ \rho_{t}$, where $\rho_{t}: x_{t} \rightarrow x_{\alpha^{-1} t}$ is obtained by deforming through the imaginary rel leaf. We may consider the mapping class of the composition $\varphi_{t} \circ \rho_{t}$ as determined by deforming $x_{t}$ through a loop in moduli space, namely a loop which follows the vertical rel leaf and then returns along the 
geodesic given by the $A$-action. Correspondingly, there is an element $\gamma_{t} \in \operatorname{Mod}(S, \Sigma)$ obtained by lifting this loop to $\mathcal{H}_{\mathrm{m}}$. The map $t \mapsto \gamma_{t}$ depends continuously on $t$, but since $\operatorname{Mod}(S, \Sigma)$ is discrete, it must be constant. By Lemma 3.9, in the case $t=\beta$ we find that $\gamma_{\beta}$ lies in the renormalization isotopy class of $x_{\beta}=q_{0}$, and hence $\varphi_{t} \circ \rho_{t}$ lies in the renormalization isotopy class of $x_{t}$. This proves statement (1) of the theorem.

We will now prove statement (2). Consider statement (a) where $t=\sum_{j=-\infty}^{k} \alpha^{-j}=\alpha^{k+2} \beta$. By 3.16 we have $x_{t}=\widetilde{g}^{k+2} x_{\beta}$. Based on our presentation of $q_{0}$, we see that $x_{t}$ has $\mathbf{g}$ cylinders whose circumferences are $\alpha^{k+2}$ times as large as those of $q_{0}$. Now consider statement (b). Here $t=\alpha^{k+2}(\beta+s)$ for some $s \in(0, \alpha)$. Using (3.17), we see that $x_{t}=\widetilde{g}^{k+2} q_{s}$. A cylinder decomposition of $q_{s}$ is described by Proposition 3.8 including the dimensions of the cylinders. The circumferences of $x_{t}$ must be $\alpha^{k+2}$ times as large. The positions of the singularities can also be determined by considering $q_{s}$, see the discussion preceding Proposition 3.8 .

3.4. Behavior of cylinders. We will now investigate the behavior of homology classes of cylinders as we move through the imaginary rel leaf $\left\{x_{t}\right\}_{t>0}$. We may identify topological objects associated to the family of surfaces $\left\{x_{t}\right\}$ using homeomorphisms $\rho$ obtained by moving through the rel leaf as in $\$ 2.4$. With a view to the applications considered in $\$ 6.3$, we will assume in this section that

$$
t(1-\alpha) \text { is not an integral power of } \alpha,
$$

which guarantees that $x_{t}$ has $\mathbf{g}+1$ horizontal cylinders; see Theorem $3.7(2)$.

For any such $t$, let

$$
C_{0}(t), C_{1}(t), \ldots, C_{\mathbf{g}}(t) \in H_{1}(S \backslash \Sigma ; \mathbb{Z})
$$

denote the homology classes of core curves of the horizontal cylinders of $x_{t}$ oriented rightward and ordered from longest to shortest circumference. From Theorem 3.7(1), we know

$$
\varphi_{*}\left(C_{i}\left(\alpha^{-1} t\right)\right)=C_{i}(t) \text { for all such } t \text { and all } i=0, \ldots, \mathbf{g},
$$

where $\varphi: S \rightarrow S$ denotes the (common) renormalization mapping class of the suspensions.

Recall from 2.5 that if $C_{i}$ is a cylinder, $C_{i}^{*} \in H^{1}(S, \Sigma ; \mathbb{Z})$ denotes the cohomology class where $C_{i}^{*}(\gamma)=\gamma \cap C_{i}$ for all $\gamma \in H_{1}(S, \Sigma ; \mathbb{Q})$. The span of these classes in $H^{1}(S, \Sigma ; \mathbb{Z})$ will be important in $\S 6$, when we study twist coordinates for analyzing the horizontal rel orbits of $x_{t}$. This span is closely connected to vertical holonomy for the family of 
surfaces $\left\{x_{t}\right\}$ as a linear map. Let $\mathbb{Q}(\alpha)+\mathbb{Q} t$ denote the $\mathbb{Q}$-vector space of expressions of the form $a+b t$ where $t$ is a free variable, $a \in \mathbb{Q}(\alpha)$ and $b \in \mathbb{Q}$. Then we can consider the vertical holonomy map of the family to be

$$
\widetilde{\operatorname{hol}_{y}}: H^{1}(S, \Sigma ; \mathbb{Q}) \rightarrow \mathbb{Q}(\alpha)+\mathbb{Q} t ; \quad \gamma \mapsto \operatorname{hol}_{y}\left(\gamma ; x_{t}\right) .
$$

Lemma 3.10. For any $t_{0}>0$ satisfying (3.20), the span over all horizontal cylinders $C$ of $x_{t_{0}}$ of the cohomology classes $C^{*} \in H^{1}(S, \Sigma ; \mathbb{Q})$ is the $\mathbf{g}+1$ dimensional $\mathbb{Q}$-subspace

$$
\left\{L \circ{\widetilde{\mathrm{hol}_{y}}}_{\mathrm{a}}: L \text { is a } \mathbb{Q} \text {-linear map } \mathbb{Q}(\alpha)+\mathbb{Q} t \rightarrow \mathbb{Q}\right\} \subset H^{1}(S, \Sigma ; \mathbb{Q}) \text {. }
$$

Proof. Let $C_{0}^{*}, \ldots, C_{\mathbf{g}}^{*}$ denote the cohomology classes to the cylinders of $x_{t_{0}}$ ordered from longest to shortest circumference as above. Let $V=\operatorname{span}_{\mathbb{Q}}\left\{C_{0}^{*}, \ldots, C_{\mathbf{g}}^{*}\right\}$, and let $W$ be the space defined in (3.23). We will first show that $W \subset V$. Let $L: \mathbb{Q}(\alpha)+\mathbb{Q} t \rightarrow \mathbb{Q}$ be $\mathbb{Q}$-linear. Because $t_{0}$ satisfies (3.20), the cylinders survive for $t$ in a neighborhood of $t_{0}$. For any $t$ in this neighborhood and any $\gamma \in H_{1}(S, \Sigma ; \mathbb{Q})$, we have

$$
L \circ \widetilde{\operatorname{hol}}_{y}(\gamma)=-\sum_{i=0}^{\mathrm{g}} L\left(h_{i}\right) C_{i}^{*}(\gamma),
$$

where $h_{i} \in \mathbb{Q}(\alpha)+\mathbb{Q} t$ denotes the height of $C_{i}$, which varies linearly with $t$. This shows $W \subset V$. To see $V=W$ it suffices to show that they have the same $\mathbb{Q}$-dimension. Observe that $W$ has $\mathbb{Q}$-dimension $\mathrm{g}+1$ because its elements are in bijection with the choice of $L$ from the dual space to $\mathbb{Q}(\alpha)+\mathbb{Q} t$. The space $V$ has $\mathbb{Q}$-dimension $\mathbf{g}+1$ when $t \in(\beta, \beta+\alpha)$ (corresponding to $q_{s}$ for $s \in(0, \alpha)$ ) by Proposition 3.8. and has this same dimension for $t_{0}$ by considering that we can find a $k \in \mathbb{Z}$ so that $\alpha^{-k} t_{0} \in(\beta, \beta+\alpha)$ and applying (3.21.

\section{Recognizing the Arnoux-Yoccoz surfaces}

In $\S 3$ we produced an imaginary rel ray $\left\{x_{r}\right\}_{r>0}$ consisting of horizontally completely periodic surfaces for each genus $\mathbf{g} \geqslant 2$. In this section we explain that $x_{r}$ limits on the Arnoux-Yoccoz surface of genus $\mathbf{g}$ as $r \rightarrow 0$ and consider consequences.

4.1. The Arnoux-Yoccoz surfaces. Here we define the Arnoux-Yoccoz surfaces in genus $\mathbf{g} \geqslant 3$. We will follow Arnoux and Yoccoz, who describe this family of surfaces as the canonical suspension of the interval exchange $\mathcal{I E}$ of $\$ 3.1$. This relies on work of Levitt, and on Thurston's classification of surface homeomorphisms [Th, FLP]. 
We recall a construction of $\$ 3.2$; $(q, \gamma)$ is a canonically labeled suspension of $\mathcal{I} \mathcal{E}$ with $q \in \mathcal{H}(\mathbf{g}-1, \mathbf{g}-1)$, then so is $(q, \hat{\gamma})$, and there is a corresponding renormalization mapping class $[\varphi]$ where $\varphi: q \rightarrow q$. See Corollary 3.4 and Definition 3.5. We need the following:

Lemma 4.1. Let $\mathbf{g} \geqslant 3$, let $(q, \gamma)$ be a suspension of $\mathcal{I} \mathcal{E}$ with $q \in$ $\mathcal{H}(\mathbf{g}-1, \mathbf{g}-1)$, and let $[\varphi]$ be the renormalization mapping class. Then $[\varphi]$ is pseudo-Anosov. Furthermore if $q$ is a measure preserving suspension then the action of $[\varphi]$ scales the vertical measured foliation of $q$ by $\alpha^{-1}$.

We include the argument here for completeness and to indicate the differences between the case of $\mathbf{g} \geqslant 3$ and $\mathbf{g}=2$ which is handled in the next subsection.

Proof. It suffices to take $(q, \gamma)$ to be a measure preserving suspension of $\mathcal{I E}$. Let $\varphi: q \rightarrow q$ be a homeomorphism which satisfies $\gamma=\varphi \circ \hat{\gamma}$ and which carries vertical leaves to vertical leaves. By definition $\varphi$ is in the renormalization mapping class.

Let $\left(\mathcal{F}^{(v)}, \mu\right)$ be the vertical foliation on $q$ together with its Lebesgue transverse measure. Observe that $\varphi\left(\mathcal{F}^{(v)}, \mu\right)=\left(\mathcal{F}^{(v)}, \alpha^{-1} \mu\right)$. This is because $\psi$ (of Theorem 3.1, used in definition of $\hat{\gamma}$ ) scales measure uniformly by a factor of $\alpha^{-1}$. This measured foliation has a representative homology class in absolute homology $H_{1}(q ; \mathbb{R})$, and thus the action $\varphi_{*}: H_{1}(q ; \mathbb{R}) \rightarrow H_{1}(q ; \mathbb{R})$ has an eigenvalue of $\alpha^{-1}$. We will see that this allows us to classify $[\varphi]$ as pseudo-Anosov in the sense of Thurston's classification of surface homeomorphisms [FLP, Theorem $9.16]$.

According to Thurston's classification of surface homeomorphisms, $[\varphi]$ is either periodic, reducible (acts periodically on an isotopy class of simple closed curves), or pseudo-Anosov.

First observe $[\varphi]$ can not be periodic, since the eigenvalue $\alpha^{-1}$ of $\varphi_{*}$ is not a root of unity.

Now suppose $[\varphi]$ was reducible. We will replicate the argument of [AY, Lemma 2]. By definition of reducibility, a positive power $\left[\varphi^{k}\right]$ leaves invariant a subsurface $S \subset q$ of lower genus. The induced action on absolute homology of the subsurface has an eigenvalue of $\alpha^{-k}$, since it preserves the intersection of the vertical measured foliation with the subsurface. We note that because $\alpha^{-1}$ is Pisot, $\alpha^{-k}$ also must have degree g. Observe that the rank of $H_{1}(S ; \mathbb{R})$ is twice the genus of $S$, which is strictly less than $2 g$. Since the action $\left.\varphi_{*}^{k}\right|_{S}$ of $\left[\varphi^{k}\right]$ on $H_{1}(S ; \mathbb{R})$ is by a integer matrix, we see that $\alpha^{-k}$ and all its $\mathbf{g}$ algebraic conjugates must be eigenvalues. Since this action is symplectic, $\alpha^{k}$ and all its $\mathbf{g}$ 
conjugates must appear as eigenvalues of $\left.\varphi_{*}^{k}\right|_{S}$ as well. Because the rank of $H_{1}(S ; \mathbb{R})$ is less than $2 g$, some of these values must coincide, meaning that $\alpha^{-k}$ is conjugate to $\alpha^{k}$. Observe that $\alpha^{-k}$ is Pisot, and the product of all its conjugates is $(-1)^{k} \alpha^{k}$. When the degree $\mathbf{g} \geqslant 3$, this coincidence is impossible. This contradicts the irreducibility of $[\varphi]$ and shows that $[\varphi]$ must be pseudo-Anosov. (This argument fails when $\mathbf{g}=2$, because $\alpha^{-2}$ is conjugate to $\alpha^{2}$.)

We will now use Lemma 4.1 to construct the Arnoux-Yoccoz surface when $\mathbf{g} \geqslant 3$. Fix a measure preserving suspension $(q, \gamma)$ and let $\varphi$ be a representative of the renormalization mapping class as in the Lemma. Let $\left(\mathcal{F}^{(v)}, \mu\right)$ denote the vertical measured foliation on $q$, so that $\varphi$ preserves $\mathcal{F}^{(v)}$, scaling $\mu$ by $\alpha^{-1}$. Let $S$ be the topological surface underlying the translation surface $q$. We identify $q$ with $S$ topologically. Since $\varphi$ is pseudo-Anosov it leaves invariant a unique second projective class of measured foliations on $S$, say the class

$$
\left\{\left(\mathcal{F}^{(h)}, c \nu\right): c>0\right\} \text {. }
$$

Integrating the pair of measures $(\mu, c \nu)$ defines a new translation structure on $S$. That is, in the charts of this alternate translation structure, the transverse measures $\mu, c \nu$ correspond respectively to the 1-forms $\mathrm{hol}_{\mathrm{x}}, \mathrm{hol}_{\mathrm{y}}$. The area of this translation surface varies linearly with $c$, and we define the Arnoux-Yoccoz surface in genus $g$ to be the surface obtained by choosing $c$ so that the area is

$$
\alpha^{\mathbf{g}} \cdot \alpha+\alpha^{\mathbf{g}-1} \cdot\left(\alpha+\alpha^{2}\right)+\alpha^{\mathbf{g}-2} \cdot\left(\alpha+\alpha^{2}+\alpha^{3}\right)+\cdots+\alpha \cdot 1 .
$$

We denote this surface by $x_{\mathrm{AY}}$. We choose this normalization of the area in order to be consistent with [AY] and [Bow1. Note that the surfaces $q_{s}$ and $x_{t}$ of $\$ 3.3$ have this area.

The two translation surfaces $q$ and $x_{\mathrm{AY}}$ are both marked by the same underlying surface $S$, and the map $q \rightarrow x_{\mathrm{AY}}$ maps vertical leaves to vertical leaves, preserving the transverse measure $\mathrm{hol}_{\mathrm{x}}$. Using this topological identification, the curve $\gamma$ on $q$ is mapped to a curve on $x_{\mathrm{AY}}$ which we also denote by $\gamma$, and we see that $\left(x_{\mathrm{AY}}, \gamma\right)$ is also a canonically labeled suspension of $\mathcal{I} \mathcal{E}$. Since $[\varphi]$ scales $\left(\mathcal{F}^{(v)}, \mu\right)$ and $\left(\mathcal{F}^{(h)}, \nu\right)$, there is a canonical affine automorphism with diagonal derivative, $\varphi_{\mathrm{AY}}: x_{\mathrm{AY}} \rightarrow x_{\mathrm{AY}}$, which is taken from the mapping class $[\varphi]$. Since $\varphi_{\text {AY }}$ scales $\left(\mathcal{F}^{(v)}, \mu\right)$ by $\alpha^{-1}$ and preserves area, it must scale $\left(\mathcal{F}^{(h)}, \nu\right)$ by $\alpha$. Thus, $D \varphi_{\mathrm{AY}}=\widetilde{g}$ with $\widetilde{g}$ as in $(3.8)$. Also note that because $\varphi_{\mathrm{AY}}$ is taken from the mapping class $[\varphi]$, it is in the renormalization isotopy class of $x_{\mathrm{AY}}$.

As a consequence of these observations we find: 
Corollary 4.2. If $(q, \gamma)$ is a suspension of $\mathcal{I E}$ with $q \in \mathcal{H}(\mathbf{g}-1, \mathbf{g}-1)$, with $\mathbf{g} \geqslant 3$, then $q$ has no vertical saddle connections.

Proof. Observe that $x_{\mathrm{AY}}$ has no vertical saddle connections because it has an affine automorphism with derivative $\widetilde{g}$. As we have seen, there is a homeomorphism $q \rightarrow x_{\mathrm{AY}}$ which carries vertical leaves to vertical leaves. So $q$ also does not have any vertical saddle connections.

Now recall the vertical rel leaf considered in $\$ 3.3$. We observe that $q_{0}=x_{\beta} \in \mathcal{H}(\mathbf{g}-1, \mathbf{g}-1)$ is a measure preserving suspension of $\mathcal{I} \mathcal{E}$ with area given by (4.1). By Corollary 4.2, $q_{0}$ has no vertical saddle connections. Therefore by Proposition 2.2, the imaginary rel leaf $\left\{x_{t}\right\}$ is defined for all $t$.

We will show that $x_{0}=x_{\mathrm{AY}}$. Recall from Theorem 3.7 that for $t>0$ we have an affine homeomorphism $\varphi_{t}: x_{\alpha^{-1} t} \rightarrow x_{t}$ with derivative $\tilde{g}$. Furthermore, if $\rho_{t}: x_{t} \rightarrow x_{\alpha^{-1} t}$ is obtained by imaginary rel deformation, then $\varphi_{t} \circ \rho_{t}$ is in the renormalization isotopy class. Now let $t \rightarrow 0^{+}$. Since $\rho_{t}$ is the map induced by $\operatorname{Rel}_{\alpha^{-1} t-t}^{(v)}$, the limit of $\rho_{t}$ is the identity map on $x_{0}$. The affine homeomorphisms converge to an affine automorphism $\varphi_{0}: x_{0} \rightarrow x_{0}$ with derivative $\widetilde{g}$. Furthermore by discreteness of the mapping class group, we know that $\left[\varphi_{0}\right]$ is in the renormalization isotopy class of $x_{0}$. Because the derivative of $\varphi_{0}$ is $\widetilde{g}$, the mapping class preserves the horizontal and vertical foliations of $x_{0}$. Up to the canonical identification between suspensions, the mapping classes $\left[\varphi_{0}\right]$ of $x_{0}$ and $\left[\varphi_{\mathrm{AY}}\right]$ of $x_{\mathrm{AY}}$ are the same. By the uniqueness of the expanding and contracting measured foliations, we see that $x_{0}$ and $x_{\mathrm{AY}}$ have the same horizontal and vertical measured foliations. Therefore, these two surfaces represent the same translation surface. We have shown:

Theorem 4.3. Let $\mathbf{g} \geqslant 3$. Let $\left\{x_{t}: t>0\right\}$ be the imaginary rel ray discussed in Theorem 3.7. Then this rel ray extends to an imaginary rel line $\left\{x_{t}: t \in \mathbb{R}\right\}$ and the surface $x_{0}$ is the Arnoux-Yoccoz surface of genus $\mathbf{g}$.

Figure 1 shows the Arnoux-Yoccoz surface $x_{\mathrm{AY}}=x_{0}$ of genus $\mathbf{g}=3$. The surfaces of higher genus have similar presentations (see [Bow1]).

Proof of Theorem 1.1. In Theorem 3.7, for each $\mathbf{g} \geqslant 2$ we exhibited an imaginary rel-ray $\left\{x_{t}: t>0\right\}$ consisting of horizontally periodic surfaces. Since $\alpha<1$, statement (2) of Theorem 3.7 shows that as $t \rightarrow+\infty$, the circumferences of the horizontal cylinders on $x_{t}$ tend uniformly to zero. Theorem 4.3 shows that for $\mathbf{g} \geqslant 3, x_{t}=\operatorname{Rel}_{t}^{(v)} x_{0}$ for $t>0$, concluding the proof. 
4.2. The noded surface in genus two. When $\mathbf{g}=2$, there is also a "canonical suspension" of $\mathcal{I E}$ as a pair of tori attached at a node. These observations on the genus 2 version of the Arnoux-Yoccoz surface are due to Bowman [Bow1, Appendix], so we will call this surface the Arnoux-Yoccoz-Bowman noded surface. We construct this surface below and then connect it to the imaginary rel ray in $\mathcal{H}(1,1)$ described in $\$ 3.3$.

Lemma 4.4. Suppose $\mathbf{g}=2$ and $(q, \gamma)$ is a suspension of $\mathcal{I} \mathcal{E}$ with $q \in \mathcal{H}(1,1)$. Then there are two vertical saddle connections on $q$ each of which pass through one of the images under $\gamma$ of the midpoints of the intervals $J_{1}$ and $J_{2}$ of (3.2). These saddle connections separate $q$ into two tori (glued along a slit), the action of $\left[\varphi^{2}\right]$ swaps the saddle connections and the tori. The action of $\left[\varphi^{2}\right]$ restricted to either torus is pseudo-Anosov.

Proof. First consider the suspension of $\mathcal{I} \mathcal{E}$ shown in the right hand side of Figure 3, where we take $\gamma$ to be a unit speed parametrization of a straightline of slope $\frac{1}{2}$ passing through the cylinder represented by the large square. The surface can be described as two tori glued along a vertical slit to form a genus 2 surface. The reglued slits form the two vertical saddle connections on the surface (shown as dotted lines in the Figure 3). The suspension is a measure preserving suspension, and the midpoints of the bottom edges (of slope $\frac{1}{2}$ ) of the smaller squares lie on the vertical leaves passing through the images under $\gamma$ of the midpoints of $J_{1}$ and $J_{2}$. We have verified the first assertion of the lemma holds for one choice of $(q, \gamma)$ with $q \in \mathcal{H}(1,1)$. This assertion follows for any choice, because the canonical homeomorphism between two suspensions sends saddle connections to saddle connections. See $\$ 2.2$.

It can be observed that the saddle connections and hence the tori are swapped by the renormalizing homeomorphism $\varphi: q \rightarrow q$. Fix one of the tori. To see that the restriction of $\varphi^{2}$ to this torus is pseudoAnosov we argue as follows. The action of $\varphi^{2}$ on each torus expands the vertical measured foliation by the quadratic irrational $\alpha^{-2}$. This is not a root of unity so the action is not periodic. Since first absolute homology is 2-dimensional, the action cannot be reducible. Thus $\varphi^{2}$ restricted to the torus is pseudo-Anosov.

We will now construct $x_{\mathrm{AYB}}$. Let $(q, \gamma)$ be a measure preserving suspension of $\mathcal{I E}$ with $q \in \mathcal{H}(1,1)$. Let $S$ be the topological surface underlying the translation structure on $q$. Let $\left(\mathcal{F}^{(v)}, \mu\right)$ be the vertical measured foliation. By the above result, there are vertical saddle 
connections in $q$ which separate $q$ into two tori. The action of $\left[\varphi^{2}\right]$ on either of the tori is pseudo-Anosov. The action of $\left[\varphi^{2}\right]$ scales $\left(\mathcal{F}^{(v)}, \mu\right)$ by $\alpha^{-2}$. Because the action $\left[\varphi^{2}\right]$ on each torus is pseudo-Anosov, there is a projective class of measured foliations on each torus which is contracted by a factor of $\alpha^{2}$. Choose the measured foliations $\left(\mathcal{F}_{1}^{(h)}, \nu_{1}\right)$ and $\left(\mathcal{F}_{2}^{(h)}, \nu_{2}\right)$ from these classes so that the areas of the tori as measured by $\mu \times \nu_{i}$ is half of the value in (4.1). Then the action of $[\varphi]$ satisfies

$$
\varphi_{*}\left(\mathcal{F}_{1}^{(h)}, \nu_{1}\right)=\left(\mathcal{F}_{2}^{(h)}, \alpha \nu_{2}\right) \text { and } \varphi_{*}\left(\mathcal{F}_{2}^{(h)}, \nu_{2}\right)=\left(\mathcal{F}_{1}^{(h)}, \alpha \nu_{1}\right) .
$$

Let $\sigma$ be one of the vertical saddle connections on $q$ in the common boundary of the two tori. Then for each $i, \varphi^{2}(\sigma)=\sigma$ and $\varphi_{*}^{2}\left(\nu_{i}\right)(\sigma)=$ $\alpha^{2} \nu_{i}(\sigma)$, so $\nu_{i}(\sigma)=0$. Let

$$
\left(\mathcal{F}^{(h)}, \nu\right)=\left(\mathcal{F}_{1}^{(h)} \cup \mathcal{F}_{2}^{(h)}, \nu_{1}+\nu_{2}\right)
$$

Then the structure on $q$ induced by the product of this horizontal transverse measure with $\left(\mathcal{F}^{(v)}, \mu\right)$ gives $S$ a noded translation structure, which we call the Arnoux-Yoccoz-Bowman noded surface $x_{\mathrm{AYB}}$. It is noded in the sense that the simple closed curve consisting of the union of the two vertical saddle connections has been collapsed to a point (it gets no transverse measure with respect to either $\mu$ or $\nu$ ).

We now summarize some results regarding a bordification of $\mathcal{H}(1,1)$. See [B, §5] for more details, and proofs of the following facts. The stratum $\mathcal{H}(1,1)$ can be viewed as a subset of the bundle of holomorphic 1-forms $(X, \omega)$ where $X$ is a Riemann surface of genus 2 (because of our conventions that singularities are labeled, in order to make this precise, one should pass to a twofold cover of this bundle). Deligne and Mumford defined a compactification of the moduli space $\mathcal{M}_{2}$ of genus 2 surfaces by adjoining moduli spaces of stable curves as boundary components of $\mathcal{M}_{2}$, and the bundle of holomorphic 1-forms on $\mathcal{M}_{2}$ can be extended to a bundle of 'stable forms' over the Deligne-Mumford compactification. An example of a stable curve in the boundary of $\mathcal{M}_{2}$ is two tori attached at a node, and a stable form over such a surface is a pair of nonzero holomorphic 1-forms on a torus with one marked point. Thus, in our notation, the union

$$
\mathcal{H}(1,1) \cup[(\mathcal{H}(0) \backslash\{0\}) \times(\mathcal{H}(0) \backslash\{0\})]
$$

inherits a topology from the bundle of stable 1-forms over the DeligneMumford compactification of $\mathcal{M}_{2}$. This topology is compatible with polygonal presentations of translation surfaces, in the following sense. Suppose $\left\{x_{t}: t>0\right\}$ is a collection of translation surfaces in $\mathcal{H}(1,1)$ which are a connected sum of two tori glued along a slit, such that the 
length of the slit goes to zero as $t \rightarrow 0+$, and the polygonal representation of each of the connected components of the complement of the slit has a Hausdorff limit of positive area. Then $\lim _{t \rightarrow 0+} x_{t}$ exists as a stable 1-form on two tori attached at a node, and is represented in $(\mathcal{H}(0) \backslash\{0\}) \times(\mathcal{H}(0) \backslash\{0\})$ by the Hausdorff limit of the polygonal presentations of the $x_{t}$.

Using this terminology we have:

Theorem 4.5. Let $\mathbf{g}=2$. Let $\left\{x_{t}: t>0\right\}$ be the imaginary rel ray discussed in Theorem 3.7 and defined by $x_{t}=\operatorname{Rel}_{t-\beta}^{(v)} q_{0}$. Then $x_{\mathrm{AYB}}=\lim _{t \rightarrow 0+} x_{t}$.

Proof. We fix $\mathbf{g}=2$ and define $\alpha, \beta, q_{0}$ via (3.1), (3.7), and $\$ 3.3$ respectively. We get $\alpha=\frac{\sqrt{5}-1}{2}$ and $\beta=1$, and the surface $q_{0}=x_{1}$ is shown in Figure 8. The surface breaks into two tori (depicted as white and gray regions) along vertical saddle connections of length 1 . We change the presentation by cutting along the dotted lines and regluing using edge identifications. The resulting surface is shown on the left side of Figure 9. The two regions shaded white and gray are two tori glued along a slit, and as we apply $\operatorname{Rel}_{t}^{(v)}$ and let $t \rightarrow-1$, the length of the slit goes to zero. By the preceding discussion we can apply $\operatorname{Rel}_{-1}^{(v)}$ to this presentation and obtain the limit of the surfaces $x_{t}$ as $t \rightarrow-1$. This collapses the vertical saddle connections resulting in the noded surface shown on the right side of Figure 9. A curve $\gamma$ representing the measure preserving suspension of $\mathcal{I E}$ is shown as a dotted line in the Figure. (This curve is in the same homotopy class as the core curve of the cylinder $C_{0}$ in the description of $q_{0}$ provided in $\$ 3.3$.) To see that $x_{0}$ is $x_{\mathrm{AYB}}$, it suffices to check that the areas match and that the renormalization mapping class of $\left(x_{0}, \gamma\right)$ is realized by an affine automorphism with derivative $\tilde{g}$. We leave these details to the reader.

Proof of Theorem 1.5. Statements (i) and (ii) follow from Theorem 3.7 , and statement (iii) follows from Theorem 4.5. Statement (iv) was proved by McMullen in [McM3, Theorem 9.3].

4.3. Genus three. In this subsection we fix $\mathbf{g}=3$. Let $x_{0}$ be the Arnoux-Yoccoz surface of genus 3. This surface admits some special symmetries:

- The surface $x_{0}$ is hyperelliptic, and the hyperelliptic involution preserves the surface's singularities [HLM, Lemma 2.1] [Bow2, $\S 1.2]$. 


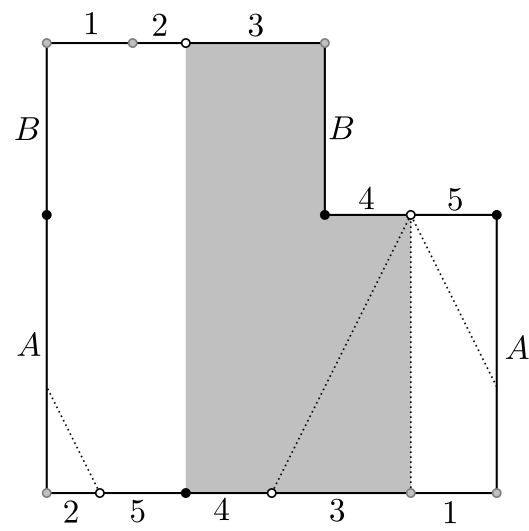

\begin{tabular}{ll} 
Label & Edge length \\
\hline $1,4,5$ & $\frac{3-\sqrt{5}}{4}$ \\
2 & $\frac{\sqrt{5}-2}{2}$ \\
3 & $\frac{\sqrt{5}-1}{4}$ \\
$A$ & $\frac{\sqrt{5}-1}{2}$ \\
$B$ & $\frac{3-\sqrt{5}}{2}$
\end{tabular}

Figure 8 . The surface $q_{0}$ with $\mathbf{g}=2$ as presented in $\$ 3.3$.
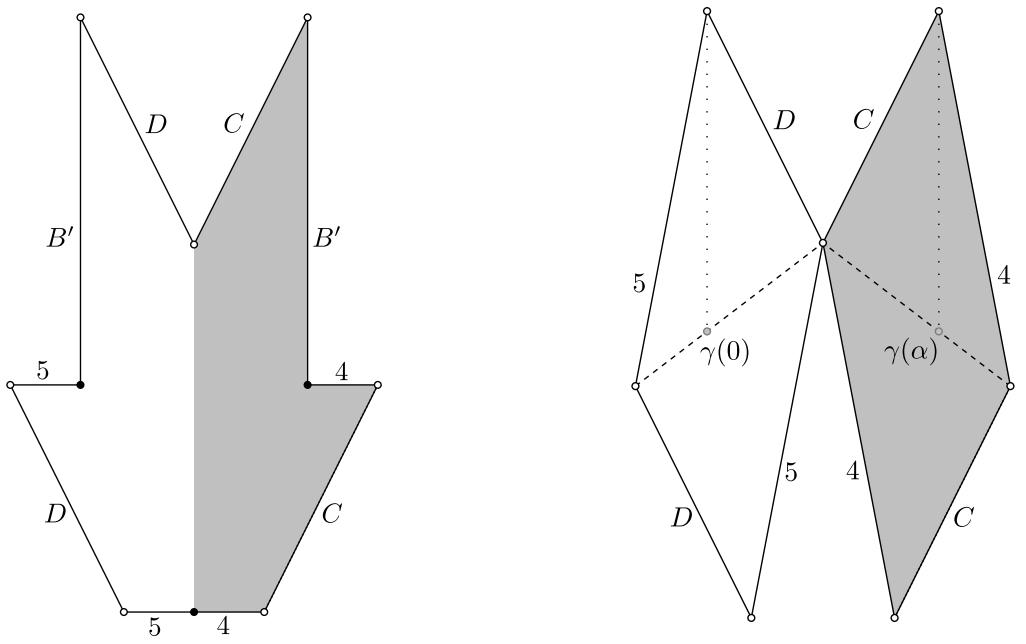

Figure 9. Left: The surface $q_{0}$ with an alternate presentation. Right: The noded surface $x_{0}=\operatorname{Rel}_{-1}^{(v)} q_{0}$ is $x_{\mathrm{AYB}}$. Edges labeled $C$ and $D$ have holonomy $\left( \pm \frac{\sqrt{5}-1}{4}, \frac{\sqrt{5}-1}{2}\right)$ and edges on the right labeled 4 and 5 have holonomy $\left( \pm \frac{3-\sqrt{5}}{4}, 1\right)$.

- The surface $x_{0}$ admits an orientation-reversing involution preserving singularities with derivative

$$
h=\left[\begin{array}{rr}
0 & \alpha \\
\alpha^{-1} & 0
\end{array}\right] \text {. }
$$

(This involution was described as $\rho_{1}^{\prime}$ acting on an affine image of $x_{0}$ in [Bow2, §2.3].) 
Proof of Corollary 1.2. The involutions above preserve the singularities and thus interact with the rel deformation as described by Proposition 2.4. In particular, for all $t \in \mathbb{R}$,

$$
-I\left(\operatorname{Rel}_{t}^{(v)} x_{0}\right)=\operatorname{Rel}_{-t}^{(v)} x_{0} \quad \text { and } \quad h\left(\operatorname{Rel}_{t}^{(v)} x_{0}\right)=\operatorname{Rel}_{\alpha t}^{(h)} x_{0}
$$

In particular, the group generated by the matrices $-I$ and $h$ acts transitively on the four horizontal and vertical rel rays leaving $x_{0}$. The conclusion of the Corollary follows.

These symmetries are a special feature of the case $\mathbf{g}=3$ :

Proposition 4.6. For $\mathbf{g} \geqslant 2$, the surface $x_{0}$ admits no nontrivial translation automorphisms. For $\mathbf{g} \geqslant 4$, it admits no involutions with derivative $-\mathrm{Id}$, and no orientation-reversing involutions with derivative $(x, y) \mapsto(x,-y)$ fixing the singular points.

Proof. The proof relies on work of Bowman [Bow1, and we will freely use Bowman's notation and results. To see that the Arnoux-Yoccoz surfaces have no translation automorphisms, note that there is no saddle connection parallel to $P_{\mathbf{g}-1} Q_{\mathbf{g}-1}$ and of equal length. The proof of Bow1, Cor. 1.6] shows that for $\mathbf{g} \geqslant 4$, the surface has no involutions with derivative $-\mathrm{Id}$. On the other hand it does admit an involution swapping singular points with derivative $(x, y) \mapsto(x,-y)$. If it had a second involution with the same derivative, fixing singular points, the composition of these two maps would be a nontrivial translation automorphism.

We will now explain Figure 2, which describes the algebraic dynamics of the rel-deformed Arnoux-Yoccoz IET. Let $x_{r}^{\prime}=\operatorname{Rel}_{r}^{(h)} x_{0}$. For $0 \leqslant$ $r<\frac{\alpha^{3}}{2}$, the surface $x_{r}^{\prime}$ can be considered to be the surface obtained from the presentation of $x_{0}$ in Figure 1 by moving the white singularity rightward by $r$. As such $x_{r}^{\prime}$ is a suspension of an interval exchange $\mathcal{I} \mathcal{E}_{r}$ defined using the same permutation as the Arnoux-Yoccoz IET viewed as an interval exchange on $[0,1)$, i.e. the permutation

$$
\left(\begin{array}{lllllll}
1 & 2 & 3 & 4 & 5 & 6 & 7 \\
2 & 5 & 4 & 7 & 6 & 3 & 1
\end{array}\right)
$$

and with the vector of lengths

$$
\left(\frac{1-\alpha}{2}, \alpha-\frac{1}{2}+r, \frac{a}{2}-r, \frac{\alpha^{2}}{2}+r, \frac{\alpha^{2}}{2}-r, \frac{\alpha^{3}}{2}+r, \frac{\alpha^{3}}{2}-r\right) .
$$


Observe that for any such $r$ and any point $p \in[0,1)$, the difference $\mathcal{I E}_{r}(p)-p$ takes one of the following six values modulo one:

$$
\pm \frac{1-\alpha}{2}, \quad \pm \frac{1-\alpha^{2}}{2}, \quad \pm \frac{1-\alpha^{3}}{2}
$$

Let $H$ be the group of rotations of $\mathbb{T}=\mathbb{R} / \mathbb{Z}$ generated by these six numbers, and let $\Gamma$ denote the Cayley graph of $H$ with respect to these six generators. As a graph $\Gamma$ is isomorphic to the graph obtained from the edges of the tiling of the plane by equilateral triangles. A choice of $p \in[0,1)$ and $r$ as above determines an embedded curve in $\Gamma$, i.e., a sequence of elements $h_{n} \in H$ so that

$$
\mathcal{I E}_{r}^{n}(p)=p+h_{n} \quad \bmod \mathbb{Z},
$$

where each $h_{n}$ is viewed as a rotation of $\mathbb{T}$. We call $\left\{h_{n}\right\}$ the arithmetic orbit of $p$.

Since each $x_{r}^{\prime}$ for $r>0$ has a vertical cylinder decomposition, the corresponding interval exchanges $\mathcal{I} \mathcal{E}_{r}$ are periodic. Based on Theorem 3.7, the periods decrease at discrete times as $r$ increases. Through an understanding of $x_{r}^{\prime}$ when $r$ is close to but slightly smaller than $\frac{\alpha^{3}}{2}$, it can be observed that the simplest such periodic orbit has period three, visiting the sequence of intervals with indices 1 then 6 then 4 . (This periodic orbit is realized by starting near the right endpoint of interval 1.) Call the cyclically ordered list 164 the orbit type of this periodic orbit. Every point of $[0,1)$ has such an orbit type under $\mathcal{I E}_{r}$ for $r$ as above: it is the cyclic sequence of interval indices hit by the orbit. For each $p$ and each $r$ for which the orbit $\mathcal{I E}_{r}^{\mathbb{Z}}(p)$ is defined, there is an open set of values $\left(p^{\prime}, r^{\prime}\right) \in \mathbb{R}^{2}$ which give rise to the same orbit type. The orbit-type determines the arithmetic orbit, because the translations applied to each interval do not vary with $r$. The arithmetic orbit corresponding to this simplest periodic orbit is a triangle, depicted in the top left of Figure 2 .

It follows from the discussion above and Theorem 3.7(1) that $\widetilde{g} x_{\alpha r}^{\prime}=$ $x_{r}^{\prime}$ for all $r$. Identifying surfaces in the real-rel leaf through rel deformations, we see that the affine homeomorphism $x_{\alpha r}^{\prime} \rightarrow x_{r}^{\prime}$ with derivative $\tilde{g}$ is in the renormalization mapping class $[\varphi]$ of $x_{0}$. Then the image un$\operatorname{der} \varphi^{-1}$ of a (homotopy class of a) cylinder $C$ in $x_{r}^{\prime}$ yields a (homotopy class of a) cylinder $C^{\prime}$ in $x_{\alpha r}^{\prime}$. Restrict to the case when $0<r<\frac{\alpha^{3}}{2}$. Then a cylinder $C$ in $x_{r}^{\prime}$ can be associated to an orbit type $O$ of $\mathcal{I} \mathcal{E}_{r}$, and the cylinder $C^{\prime}$ in $x_{\alpha r}^{\prime}$ is associated to a different orbit type $O^{\prime}$. Using a natural Markov partition for the action of $\varphi^{-1}$ on $x_{0}$, it can be observed that $O^{\prime}$ can be derived from $O$ by applying the following 
"substitution" (which makes sense for cyclic words):

$$
\begin{gathered}
1 \mapsto 34,2 \mapsto 34,4 \mapsto 16,5 \mapsto 17,6 \mapsto 2,7 \mapsto 3 \\
3 \mapsto \begin{cases}35 & \text { if the number preceding } 3 \text { was } 4 \text { or } 7, \\
15 & \text { if the number preceding } 3 \text { was } 3 \text { or } 6 .\end{cases}
\end{gathered}
$$

It follows from Theorem 3.7 that the orbit of 164 under this substitution of gives all orbit types of periodic orbits of IETs in the family $\left\{\mathcal{I E}_{r}\right.$ :

$\left.0 \leqslant r<\frac{\alpha^{3}}{2}\right\}$. So the simplest orbit types of such periodic orbits are

164, 34216, 151634342 and 34173421516351634

corresponding to the four arithmetic orbits depicted on the top left side of Figure 2,

The substitution of 4.2 has the Tribonacci substitution

$$
a \mapsto a b ; \quad b \mapsto a c ; \quad c \mapsto a
$$

as a factor obtained by mapping 1,2 and 3 to $a$, mapping 4 and 5 to $b$ and mapping 6 and 7 to $c$. We speculate that after passing to a subsequence, the arithmetic orbits (as depicted in Figure 2) converge up to rescaling and a uniform affine coordinate change to Rauzy's fractal [Ra] in the Hausdorff topology. As curves, these rescaled paths likely converge to the curve defined by Arnoux's semi-conjugation [Arn2] between the Arnoux-Yoccoz IET and the toral rotation associated to Rauzy's fractal. We hope to investigate these relations in future work.

\section{Results of Eskin-Mirzakhani-Mohammadi, Consequences AND SUBSEQUENT DEVELOPMENTS}

Recent breakthrough results of Eskin, Mirzakhani and Mohammadi EMi, EMiMo] give a wealth of information about orbit-closures for the actions of $G$ and $P$ on strata of translation surfaces. The following summarizes the results which we will need in this paper:

Theorem 5.1. Let $x$ be a translation surface in a stratum $\mathcal{H}$, and let $\mathcal{H}_{0}$ be the subset of the connected component of $\mathcal{H}$ containing $x$, and consisting of surfaces whose area is the same area as the area of $x$. Then

$$
\overline{G x}=\overline{P x}=\mathcal{M} \cap \mathcal{H}_{0},
$$

where $\mathcal{M}$ is an immersed submanifold of $\mathcal{H}$ of even (real) dimension which is cut out by linear equations with respect to period coordinates, and $\mathcal{M} \cap \mathcal{H}_{0}$ is the support of a finite smooth invariant measure $\mu$. Moreover

$$
\frac{1}{T} \int_{0}^{T} \int_{0}^{1}\left(g_{t} u_{s}\right)_{*} \delta_{x} d s d t \rightarrow_{T \rightarrow \infty} \mu
$$


where $\delta_{x}$ is the Dirac measure at $x$ and the convergence is weak- ${ }^{*}$ convergence in the space of probability measures on $\mathcal{H}$.

5.1. Surfaces whose horocycle orbit-closure contains their $G$ orbit. Recall that Proposition 1.7 claimed that if $x$ periodic under $g_{t}$, then $\overline{U x}=\overline{V x}=\overline{G x}$.

Proof of Proposition 1.7. We prove for $U$, the proof for $V$ being similar. Suppose $g_{p_{0}} x=x$, where $p_{0}>0$ is the period for the closed geodesic. Let $\mu$ be the smooth $G$-invariant measure with $\overline{G x}=\operatorname{supp} \mu$. By (5.1),

$$
\begin{aligned}
\mu & =\lim _{m \rightarrow \infty} \frac{1}{m p_{0}} \int_{0}^{m p_{0}} \int_{0}^{1}\left(g_{t} u_{s}\right)_{*} \delta_{x} d s d t \\
& =\lim _{m \rightarrow \infty} \frac{1}{m p_{0}} \int_{0}^{p_{0}} \sum_{i=0}^{m-1} \int_{0}^{1}\left(g_{i p_{0}+p} u_{s}\right)_{*} \delta_{x} d s d p .
\end{aligned}
$$

For each $p \in\left[0, p_{0}\right)$ we write

$\nu_{p, m}=\frac{1}{m} \sum_{i=0}^{m-1} \int_{0}^{1}\left(g_{i p_{0}+p} u_{s}\right)_{*} \delta_{x} d s=\frac{1}{m} \sum_{i=0}^{m-1} \frac{1}{e^{2\left(i p_{0}+p\right)}} \int_{0}^{e^{2\left(i p_{0}+p\right)}}\left(u_{s} g_{p}\right)_{*} \delta_{x} d s$,

where we have used the commutation relations $g_{\tau} u_{s}=u_{e^{2 \tau} s} g_{\tau}$ and the fact that $g_{i p_{0}} x=x$ for each $i$. Then the right hand-side of (5.2) is $\lim _{m \rightarrow \infty} \frac{1}{p_{0}} \int_{0}^{p_{0}} \nu_{p, m} d p$. Let $\nu_{0, m}$ be the measure corresponding to $p=$ 0 , then for any $p, \nu_{p, m}=g_{p *} \nu_{0, m}$. Take a subsequence $\left\{m_{j}\right\}$ along which $\nu_{0, m_{j}}$ converges to a measure $\nu$ on $\mathcal{H}($ where $\nu(\mathcal{H}) \leqslant 1)$. Then $\nu_{p, m_{j}} \rightarrow_{j \rightarrow \infty} g_{p *} \nu$. The right hand side of (5.3) shows that $\nu$ is $U$ invariant and therefore so is each $g_{p *} \nu$, and by (5.2) we have $\mu=$ $\frac{1}{p_{0}} \int_{0}^{p_{0}} g_{p *} \nu d p$ (and in particular $\nu(\mathcal{H})=1$ ). Since $\mu$ is $G$-ergodic, by the Mautner property (see e.g. [EW]) it is $U$-ergodic. This implies that $g_{p *} \nu=\mu$ for almost every $p$, and (since $\mu$ is $\left\{g_{t}\right\}$-invariant), $\nu=\mu$. These considerations are valid for every convergent subsequence of the sequence $\nu_{0, m}$ and hence $\nu_{0, m} \rightarrow_{m \rightarrow \infty} \mu$. Since $\nu_{0, m}$ is obtained by averaging over the $U$-orbit of $x$, the orbit $U x$ is dense in $\operatorname{supp} \mu=\overline{G x}$, i.e. $\overline{U x}=\overline{G x}$.

\subsection{Results of Hubert-Lanneau-Möller, and their extension.} In [HLM], Hubert, Lanneau and Möller determined $\overline{G x_{0}}$ for the ArnouxYoccoz surface $x_{0}$ in the case $\mathbf{g}=3$. The proof of [HLM] preceded the recent breakthroughs of [EMiMo] discussed above. In this section, using [EMiMo and ideas of Alex Wright, we show how to extend the results of [HLM] to arbitrary $\mathbf{g} \geqslant 3$. For further background we refer the reader to the article of Wright [Wr2] which surveys the theory 
of translation surfaces taking into account the results of [EMiMo] and ensuing work.

A set $\mathcal{M}$ as in Theorem 5.1 will be called an affine invariant manifold. Note that we can recover $\mathcal{M}$ from $x$ as dilations of surfaces in $\overline{G x}$, or as the orbit-closure of $x$ under the group $\mathrm{GL}_{2}^{\circ}(\mathbb{R})$ (the connected component of the identity in the group of invertible $2 \times 2$ matrices). By choosing a marking, lifting to $\mathcal{H}_{\mathrm{m}}$, and using period coordinates, the tangent space to $\mathcal{M}$ at a point can be identified using period coordinates with a subspace of $H^{1}\left(S, \Sigma ; \mathbb{R}^{2}\right)$. Moreover if we identify $\mathbb{R}^{2} \cong \mathbb{C}$ in the natural way, then this subspace is a $\mathbb{C}$-linear subspace of $H^{1}(S, \Sigma ; \mathbb{C}$ ) (see [EMiMo, AEM]). The field of definition of $\mathcal{M}$ is the smallest field $k$ such that for any $x \in \mathcal{M}$ and any identification of $T_{x}(\mathcal{M})$ with a subspace $W \subset H^{1}\left(S, \Sigma ; \mathbb{R}^{2}\right)$, using period coordinates, $W$ can be defined by linear equations with coefficients in $k$. The following summarizes results of Avila, Eskin, Filip, Möller and Wright (see [AEM, Wr1, F] and the references therein).

Theorem 5.2. The field of definition of $\mathcal{M}$ is the intersection of the holonomy fields of all surfaces in $\mathcal{M}$. It is a totally real field.

Let $\mathcal{M}$ be an affine invariant manifold, contained in a stratum $\mathcal{H}$ of surfaces of genus $\mathbf{g}$, and let Res be the map as in (2.6). We will say that $\mathcal{M}$ is of full rank in $\mathcal{H}$ if the restriction of Res to the tangent space to $\mathcal{M}$ is surjective, that is if $\operatorname{dim}_{\mathbb{C}} \operatorname{Res}(W)=2 \mathbf{g}$, where $W \subset$ $H^{1}\left(S, \Sigma ; \mathbb{R}^{2}\right)$ is the subspace corresponding to $T_{x}(\mathcal{M})$ for some $x \in \mathcal{M}$ using some marking. Similarly we will say that $\overline{G x_{0}}$ is of full rank if the corresponding affine invariant manifold $\mathcal{M}$ is of full rank.

Note that if $\mathcal{M}$ is of full rank then

$$
\operatorname{dim}_{\mathbb{C}} \mathcal{M} \geqslant \operatorname{dim}_{\mathbb{C}} \mathcal{H}-\operatorname{dim}_{\mathbb{C}} \mathfrak{R},
$$

where $\mathfrak{R}$ is as in (2.7). We will prove the following:

Theorem 5.3. Let $x$ be a translation surface of genus $\mathbf{g}$ which admits a pseudo-Anosov map such that the holonomy field of $x$ is of degree $\mathbf{g}$ and does not contain any totally real subfields other than $\mathbb{Q}$ (and in particular is not itself totally real). Then $\overline{G x}$ is of full rank.

Proof (Alex Wright, cf. [Wr1], Cor. 8.1). By Theorem 5.2, the field of definition of $\mathcal{M}$ is totally real and contained in the holonomy field of $x$. By assumption this implies that the field of definition is $\mathbb{Q}$.

Choosing $x_{1} \in \mathcal{M}$ and a marked surface $\mathbf{x}_{1} \rightarrow x_{1}$, let $W \subset H^{1}\left(S, \Sigma ; \mathbb{R}^{2}\right)$ be the subspace tangent to $\pi^{-1}(\mathcal{M})$ at $\mathbf{x}_{1}$. Let $V=\operatorname{Res}(W)$, so that when identifying $H^{1}\left(S ; \mathbb{R}^{2}\right) \cong H^{1}(S ; \mathbb{C})$, we have that $V$ is a $\mathbb{C}$-vector subspace of $H^{1}(S ; \mathbb{C})$. By Wr1, Thm. 5], $V$ is also defined over $\mathbb{Q}$. 
Since $V \subset H^{1}(S ; \mathbb{C})$ and $\operatorname{dim} H^{1}(S ; \mathbb{C})=2 \mathbf{g}$, it suffices to show that $\operatorname{dim}_{\mathbb{C}} V \geqslant 2 \mathrm{~g}$. Let $H^{1}(S ; \mathbb{C})^{(\mathrm{st})}$ be the subspace of $H^{1}(S ; \mathbb{C})$ generated by the 1 -forms $\operatorname{hol}_{\mathrm{x}}(x), \operatorname{hol}_{\mathrm{y}}(x)$. This is a 2-dimensional complex linear subspace defined over the holonomy field $k$ of $x$. It is contained in $V$ because $\mathcal{M}$ is $G$-invariant. We can find a basis $v_{1}, v_{2}$ of $H^{1}(S ; \mathbb{R})^{\text {(st) }}$ consisting of vectors with coefficients in $k$. The images of these vectors under the field embeddings $k \rightarrow \mathbb{C}$ are contained in $V$ because $V$ is defined over $\mathbb{Q}$. According to [Wr1, Thm. 1.5] (following arguments developed by Möller [Mö] for lattice surfaces), these vectors are linearly independent, and so we find that $V$ contains $2 \mathrm{~g}$ linearly independent vectors. That is, $\operatorname{dim}_{\mathbb{C}} \mathcal{M} \geqslant 2 \mathrm{~g}=\operatorname{dim}_{\mathbb{C}} H^{1}(S ; \mathbb{C})$, which shows that $\mathcal{M}$ is of full rank.

Recall Theorem 1.8, proved in Appendix A as Corollary A.8;

Theorem 5.4 (Bary-Soroker, Shusterman and Zannier). Let $\mathbf{g} \geqslant 3$ and let $\alpha$ be the unique real number in $[0,1]$ satisfying $\alpha+\cdots+\alpha^{\mathbf{g}}=1$. Then $\mathbb{Q}(\alpha)$ contains no totally real subfields other than $\mathbb{Q}$.

Corollary 5.5. Let $x_{0}$ be the Arnoux-Yoccoz surface in genus $\mathbf{g} \geqslant 3$. Then $\overline{G x_{0}}$ is of full rank.

Proof. Let $k=\mathbb{Q}(\alpha)$. Since $x_{0}$ admits a pseudo-Anosov diffeomorphism with derivative $\widetilde{g}$, its holonomy field is $\mathbb{Q}\left(\alpha+\alpha^{-1}\right)$ [KS, Theorem 28]. In particular the holonomy field of $x_{0}$ is contained in $k$. (These fields actually coincide, that is $\mathbb{Q}\left(\alpha+\alpha^{-1}\right)=\mathbb{Q}(\alpha)$, but we will not need this fact.) According to Theorem 1.8, $k$ has no totally real subfields other than $\mathbb{Q}$ and hence neither does the holonomy field of $x_{0}$. By Theorem 5.3, $\overline{G x_{0}}$ is of full rank.

Remark 5.6. In $[\mathrm{HLM}]$, the orbit-closure $\overline{G x_{0}}$ is determined explicitly in case $\mathbf{g}=3$ : it is the subset of hyperelliptic surfaces in $\mathcal{H}^{\text {odd }}(2,2)$. In work of Mirzakhani and Wright [MiWr], it is shown that the only full rank sub-loci of $\mathcal{H}(\mathbf{g}-1, \mathbf{g}-1)$ for $\mathbf{g} \geqslant 3$ arise from the space of quadratic differentials $\mathcal{Q}\left(\mathbf{g}-2, \mathbf{g}-2,-1^{2 \mathbf{g}}\right)$ by a double cover construction. In particular the only proper affine invariant submanifold of full rank consists of surfaces with a hyper-elliptic involution. As we have seen in Proposition 4.6, for $\mathbf{g} \geqslant 4$ the Arnoux-Yoccoz surface $x_{0}$ has no involutions with derivative - $\mathrm{Id}$, and therefore Corollary 5.5 and the result of [MiWr] imply that when $\mathbf{g} \geqslant 4, G x_{0}$ is dense in its stratum component, proving Theorem 1.9.

We will need the following:

Proposition 5.7. Suppose $\mathcal{M}$ is an affine invariant manifold that is of full rank in a stratum $\mathcal{H}$. For any $x \in \mathcal{H}$ let $\mathcal{H}_{0}$ be the subset of the 
connected component of $\mathcal{H}$ containing $x$, consisting of surfaces whose area coincides with that of $x$. Let $\mathcal{M}_{0}=\mathcal{M} \cap \mathcal{H}_{0}$. Then the subset

$$
\mathcal{U}=\left\{\operatorname{Rel}^{u}(z): z \in \mathcal{M}_{0}, u \in \mathfrak{R}, \operatorname{Rel}^{u}(z) \text { is defined }\right\}
$$

contains an open subset of $\mathcal{H}_{0}$ and is dense in $\mathcal{H}_{0}$.

Proof. Since $\mathcal{M}$ is of full rank, $W \oplus \Re=H^{1}\left(S, \Sigma ; \mathbb{R}^{2}\right)$ (where as above, $W$ is the subspace above identified with the tangent space to $\mathcal{M})$. Since the map $(z, u) \mapsto \operatorname{Rel}^{u}(z)$ is defined in an open set in $\mathcal{H} \times \mathfrak{R}$, for any $z \in \mathcal{M}$ there are open neighborhoods $U_{1} \subset \mathcal{M}$ of $z$ and $U_{2} \subset \mathfrak{R}$ of 0 such that the map

$$
U_{1} \times U_{2} \rightarrow \mathcal{H}, \quad\left(z^{\prime}, u\right) \mapsto \operatorname{Rel}^{u}\left(z^{\prime}\right)
$$

is a homeomorphism onto its image. Since $\mathcal{M}$ has full rank, the image contains an open subset of $\mathcal{H}$. Moreover the same is true if we replace everywhere $\mathcal{M}$ with $\mathcal{M}_{0}$ and $\mathcal{H}$ with $\mathcal{H}_{0}$. In order to show that $\mathcal{U}$ is dense, by ergodicity of the $G$-action on $\mathcal{H}_{0}$ it suffices to show that $\mathcal{U}$ is $G$-invariant. Let $z_{2}=\operatorname{Rel}^{u}\left(z_{1}\right) \in \mathcal{U}$, where $u \in \mathfrak{R}$ and $z_{1} \in \mathcal{M}_{0}$, and let $g \in G$. Then $g z_{1} \in \mathcal{M}_{0}$ since $\mathcal{M}_{0}$ is $G$-invariant, and $g z_{2}=\operatorname{Rel}^{g u}\left(g z_{2}\right)$ is defined and contained in $\mathcal{U}$ by Proposition 2.4. This completes the proof.

As above $\mathcal{H}_{0}$ denote the subset of the component of $\mathcal{H}(\mathbf{g}-1, \mathbf{g}-1)$ containing $x_{0}$ and consisting of surfaces with the same area as $x_{0}$. Combining Proposition 5.7, Corollary 5.5 and Proposition 1.7 we obtain:

Corollary 5.8. For $\mathbf{g} \geqslant 3, \overline{U x_{0}}$ is an affine invariant manifold of full rank, and the only closed subset of $\mathcal{H}_{0}$ containing $U x_{0}$ and invariant under the rel foliation is $\mathcal{H}_{0}$.

\section{Minimal tori FOR THE HOROCYCLE FLOW AND REAL-REL}

In this section we give basic information about rel leaves of periodic surfaces, and use this to complete the proof of Theorem 1.4. While the results below are elementary, they are of independent interest and we formulate them in greater generality than we need.

6.1. Twist coordinates for completely periodic surfaces. Let $\mathbf{x}$ be a marked translation surface with a non-empty labeled singular set $\Sigma$, and suppose $\mathbf{x}$ is completely periodic in the horizontal direction. Then $\mathbf{x}$ admits a decomposition into horizontal cylinders, and the surface can be recovered by knowing some related combinatorial data and some geometric parameters. Denote the horizontal cylinders by $C_{1}, \ldots, C_{m}$. A separatrix diagram for a horizontal cylinder decomposition consists of the ribbon graph of right-pointing horizontal 
saddle connections forming the union of boundaries of cylinders, whose vertices are given by $\Sigma$ (with orientation at vertices induced by the translation surface structure on a neighborhood of the singular point), and an indication of which pairs of circles in the diagram bound each cylinder $C_{i}$. For more information see [KoZo], where this notion was introduced. We add more combinatorial information by selecting for each horizontal cylinder $C_{i}$ an upward-oriented saddle connection $\sigma_{i}$ whose image is inside $C_{i}$ and which joins the bottom boundary component of $C_{i}$ to the top boundary component. The union of the separatrix diagram and the saddle connections $\sigma_{i}$ still has a ribbon graph structure induced by the surface. The complete combinatorial data for our cylinder decomposition consists of a labeling of cylinders, and the ribbon graph which is the union of the separatrix diagram and the saddle connections $\sigma_{i}, i=1, \ldots, m$.

Fixing the number and labels of cylinders and the combinatorial data above, the marked translation surface structure on $\mathbf{x}$ is entirely determined by the following parameters: the circumferences $c_{1}, \ldots, c_{m} \in \mathbb{R}_{>0}$ of the cylinders; the lengths $\ell_{1}, \ldots, \ell_{n} \in \mathbb{R}_{>0}$ of the horizontal saddle connections along the boundaries of the cylinders; and the holonomies

$$
\operatorname{hol}\left(\sigma_{i}, \mathbf{x}\right)=\left(x_{i}, y_{i}\right) \in \mathbb{R} \times \mathbb{R}_{>0}
$$

of each saddle connection $\sigma_{i}$ for $i=1, \ldots, m$. Observe that each $y_{i}$ records the height of the cylinder $C_{i}$.

We call the numbers $x_{i}$ the twist parameters. Observe that we can vary the twist parameters at will. That is, given $\mathbf{x}$ as above, there is a map

$$
\widetilde{\Phi}: \mathbb{R}^{m} \rightarrow \mathcal{H}_{\mathrm{m}} ; \quad\left(\hat{x}_{1}, \ldots, \hat{x}_{m}\right) \mapsto \hat{\mathbf{x}}
$$

where $\hat{\mathbf{x}}$ is the surface built to have the same parameters as $\mathbf{x}$ except with twist parameters given by $\hat{x}_{1}, \ldots, \hat{x}_{m}$, and where $\mathcal{H}_{\mathrm{m}}$ is the space of marked translation surfaces structures modeled on the same surface and singularity set as $\mathbf{x}$. Let $\pi: \mathcal{H}_{\mathrm{m}} \rightarrow \mathcal{H}$ be the natural projection. The image of $\pi \circ \widetilde{\Phi}$ is the set of all translation surfaces which have a horizontal cylinder decomposition with the same combinatorial data as $x=\pi(\mathbf{x})$, and the same parameters describing cylinder circumferences, lengths of horizontal saddle connections, and heights of cylinders. We refer to this set as the horizontal twist space at $x$, and denote it by $\mathcal{H} \mathcal{T}_{x}$. We wish to explicitly parameterize this space.

Let $x_{1}, \ldots, x_{m}$ denote the twist parameters for $\mathbf{x}$, and choose a second set of twist parameters $\hat{x}_{1}, \ldots, \hat{x}_{m}$. Then $\hat{\mathbf{x}}$ can be obtained from $\mathbf{x}$ by slicing each cylinder $C_{i}$ along a geodesic core curve and regluing so that 
the right side has moved right by $\hat{x}_{i}-x_{i}$. Thus for each $\gamma \in H_{1}(S, \Sigma ; \mathbb{Z})$,

$$
\operatorname{hol}(\gamma, \hat{\mathbf{x}})=\operatorname{hol}(\gamma, \mathbf{x})+\sum_{i=1}^{m}\left(\left(\hat{x}_{i}-x_{i}\right)\left(\gamma \cap C_{i}\right), 0\right)
$$

where $\cap$ from $\gamma \cap C_{i}$ denotes the algebraic intersection pairing. Recall from 2.5 that $C_{i}^{*} \in H^{1}(S, \Sigma ; \mathbb{Z})$ to denotes the cohomology class defined by $C_{i}^{*}(\gamma)=\gamma \cap C_{i}$. We have observed:

Proposition 6.1. The map $\widetilde{\Phi}$ is an affine map whose derivative is

$$
D \widetilde{\Phi}\left(\frac{\partial}{\partial x_{i}}\right)=\left(C_{i}^{*}, 0\right) \in H^{1}\left(S, \Sigma ; \mathbb{R}^{2}\right), \quad i=1, \ldots, m .
$$

Recall that $\widetilde{\Phi}$ defined in 6.2 maps vectors to elements of $\mathcal{H}_{\mathrm{m}}$, i.e. marked surfaces. Since holonomies of the saddle connections $\sigma_{i}$ distinguish surfaces in the image, $\widetilde{\Phi}$ is injective. However, the map $\pi \circ \widetilde{\Phi}: \mathbb{R}^{m} \rightarrow \mathcal{H}$ is certainly not injective. In the space $\mathcal{H}$ we consider translation surfaces equivalent if they differ by the action of an element of $\operatorname{Mod}(S, \Sigma)$. Let $\mathrm{M}(\mathbf{x}) \subset \operatorname{Mod}(S, \Sigma)$ denote the subgroup consisting of equivalence classes of orientation preserving homeomorphisms of $\mathbf{x}$ such that:

- Each cylinder $C_{i}$ is mapped to a cylinder $C_{j}$ of the same circumference and height.

- Each horizontal saddle connection is mapped to a horizontal saddle connection of the same length, respecting the orientation.

Note that each element of $\mathrm{M}(\mathbf{x})$ preserves the image of $\widetilde{\Phi}$, and that distinct twist parameters yield the same surface in $\mathcal{H}$ if and only if they differ by an element of $\mathrm{M}(\mathbf{x})$. In light of Proposition 6.1, $\mathrm{M}(\mathbf{x})$ pulls back to an affine action on the twist parameters, and we obtain an affine homeomorphism

$$
\Phi: \mathbb{R}^{m} / \mathrm{M}(\mathbf{x}) \rightarrow \mathcal{H} \mathcal{T}_{x} \subset \mathcal{H}
$$

Some elements of the subgroup $\mathrm{M}(\mathbf{x})$ are clear. Each Dehn twist $\tau_{i} \in \operatorname{Mod}(S, \Sigma)$ in each horizontal cylinder $C_{i}$ lies in $\mathrm{M}(\mathbf{x})$. The action of $\tau_{i}$ on twist parameters just affects the twist parameter $x_{i}$ of $C_{i}$ and has the effect of adding $c_{i}$, the circumference of $C_{i}$. The multi-twist subgroup $\mathrm{M}_{0}(\mathbf{x})=\left\langle\tau_{1}, \ldots, \tau_{m}\right\rangle$ of $\mathrm{M}(\mathbf{x})$ is isomorphic to $\mathbb{Z}^{m}$. Moreover, $\mathrm{M}(\mathbf{x})$ acts by permutations on the horizontal saddle connections, and $\mathrm{M}_{0}(\mathbf{x})$ is the kernel of this permutation action, and hence is normal and of finite index in $\mathrm{M}(\mathbf{x})$. Thus we have the following short exact sequence of groups

$$
\{1\} \rightarrow \mathrm{M}_{0}(\mathbf{x}) \rightarrow \mathrm{M}(\mathbf{x}) \rightarrow \Delta \rightarrow\{1\}
$$


where $\Delta$ is a subgroup of the group of permutations of the horizontal saddle connections. We set $\mathbb{T}=\mathbb{R}^{m} / \mathrm{M}_{0}(\mathbf{x}) \cong \prod_{i=1}^{m} \mathbb{R} / c_{i} \mathbb{Z}$ (an $m$ dimensional torus). By normality, the action of $\mathrm{M}(\mathbf{x})$ on $\mathbb{R}^{m}$ descends to an action on $\mathbb{T}$ which factors through an action of $\Delta$ via $(6.6)$. Thus we have the sequence of covers

$$
\mathbb{R}^{m} \rightarrow \mathbb{T} \rightarrow \mathbb{R}^{m} / \mathrm{M}(\mathbf{x}) \cong \mathbb{T} / \Delta
$$

We see that $\mathcal{H} \mathcal{T}_{x}$ is isomorphic to the quotient of a torus by a finite group of linear automorphisms. When $\Delta$ is trivial, we actually have $\mathbb{R}^{m} / \mathrm{M}(\mathbf{x})=\mathbb{T}$. Thus the following holds:

Proposition 6.2. Suppose that the horizontal cylinders of $x$ have distinct circumferences or distinct heights, and that each cylinder has a saddle connection on its boundary whose length is distinct from the lengths of other saddle connections on the boundary. Then $\mathrm{M}(\mathbf{x})=$ $\mathrm{M}_{0}(\mathbf{x})$ and therefore $\Phi: \mathbb{T} \rightarrow \mathcal{H} \mathcal{T}_{x}$ is an isomorphism of affine manifolds.

Remark 6.3. The above discussion equips the quotient $\mathcal{H} \mathcal{T}_{x} \cong \mathbb{T} / \Delta$ with the structure of an affine orbifold, since it is the quotient of $\mathbb{T}$ by the action of a finite group of affine automorphisms $\Delta$. For an example in which $\Delta$ is nontrivial and $\mathcal{H T}_{x}$ is not a torus, let $x$ be the Escher staircase surface obtained by cyclically gluing $2 m$ squares (see e.g. [LW, Figure 3]). The surface has $m$ parallel cylinders, the torus $\mathbb{T}$ is isomorphic to $(\mathbb{R} / \mathbb{Z})^{m}$, and the group $\Delta$ is a cyclic group generated by a homeomorphism of the surface which goes one step up the staircase. The group $\Delta$ acts on $(\mathbb{R} / \mathbb{Z})^{m}$ by cyclic permutations of coordinates.

Remark 6.4. The maps (6.2) and (6.5) were used in [SW] in order to analyze the horocycle flow on completely periodic surfaces, but the case in which $\Delta$ is nontrivial was overlooked. Nevertheless [SW, Prop. 4] is true as stated. Namely, in order to prove this one can work in a finite cover $\mathcal{H}^{\prime}$ of $\mathcal{H}$ in which $\mathbb{T}$ embeds, analyzing the closure of the $U$-orbit in $\mathcal{H}^{\prime}$ via the arguments used in [SW, Proof of Prop. 4]. Then, using the fact that elements of $\mathrm{M}(\mathbf{x})$ preserve circumferences and heights of cylinders which they permute, it is not hard to show that the validity of [SW, Prop. 4] in $\mathcal{H}^{\prime}$ implies that the same statement is valid in $\mathcal{H}$.

6.2. Real-rel flow in twist coordinates. Now we will specialize to the setting where $\mathbf{x} \in \mathcal{H}_{\mathrm{m}}$ has two singularities, which we label by the symbols $\bullet$ and $\circ$. Figure 6 shows an example. We continue to suppose that the horizontal direction is completely periodic, but now we will also assume that $\mathbf{x}$ admits no horizontal saddle connections joining distinct singularities. That is, each boundary edge of each horizontal 
cylinder contains only one of the two singularities. We will order the cylinders so that $C_{1}, \ldots, C_{k}$ have the singularity $\circ$ on their bottom and the singularity $\bullet$ on the top, so that $C_{k+1}, \ldots, C_{\ell}$ have $\bullet$ on the bottom and $\circ$ on the top, and $C_{\ell+1}, \ldots, C_{m}$ have the same singularity on both boundary components.

We observe that the real-rel flow applied to a surface in the horizontal twist space can be viewed as only changing the twist parameters. Concretely, $\operatorname{Rel}_{r}^{(h)}$ decreases the twist parameters of cylinders $C_{1}, \ldots, C_{k}$ by $r$, increases the twist parameters of $C_{k+1}, \ldots, C_{\ell}$ by $r$, and does not change the twist parameters of $C_{\ell+1}, \ldots, C_{m}$. Therefore we find:

Proposition 6.5. The horizontal twist space $\mathcal{H}_{x}$ is invariant under $\mathrm{Rel}^{(h)}$. Define

$$
\vec{w} \in \mathbb{R}^{m}, \quad w_{i}= \begin{cases}-1 & \text { if } i \leqslant k \\ 1 & \text { if } k<i \leqslant \ell, \\ 0 & \text { if } i>\ell .\end{cases}
$$

Then the straightline flow

$$
F_{\vec{w}}^{r}: \vec{x} \mapsto \vec{x}+r \vec{w}
$$

on $\mathbb{R}^{m}$ induces a well-defined straightline flow on $\mathbb{R}^{m} / \mathrm{M}(\mathbf{x})$ and $\Phi$ is a topological conjugacy from this flow to the restriction of $\operatorname{Rel}_{r}^{(h)}$ to $\mathcal{H} \mathcal{T}_{x}$; that is $\Phi \circ F_{\vec{w}}^{r}=\operatorname{Rel}_{r}^{(h)} \circ \Phi$ for every $r$.

Proof. In the case $\mathrm{M}(\mathbf{x})=\mathrm{M}_{0}(\mathbf{x})$, the horizontal twist space $\mathcal{H} \mathcal{T}_{x}$ is isomorphic to the torus $\mathbb{T}$ and it is straightforward to check that the effect of applying $\operatorname{Rel}_{r}^{(h)}$ on the twist coordinates is exactly $x_{i} \mapsto x_{i}+r w_{i}$, giving the required conjugacy. In the general case we need to show that the action of $F_{\vec{w}}^{r}$ and of the group $\Delta$ on $\mathbb{T}$ commute; indeed this will imply both that the action of $F_{\vec{w}}^{r}$ on $\mathbb{T} / \Delta \cong \mathcal{H} \mathcal{T}_{x}$ is welldefined, and that $\Phi$ intertwines the straightline flow $F_{\vec{w}}^{r}$ on $\mathbb{R}^{m} / \mathrm{M}(\mathbf{x})$ with the real-rel flow $\operatorname{Rel}_{r}^{(h)}$ on $\mathcal{H} \mathcal{T}_{x}$. The definition of the $w_{i}$ implies that $F_{\vec{w}}^{r}$ and $\Delta$ commute provided the permutation action of $\mathrm{M}(\mathbf{x})$ on the horizontal cylinders preserves each of the three collections of cylinders $\left\{C_{1}, \ldots, C_{k}\right\},\left\{C_{k+1}, \ldots, C_{\ell}\right\},\left\{C_{\ell+1}, \ldots, C_{m}\right\}$; this in turn follows from our assumption that singularities are labeled, and the definition of $\mathrm{M}(\mathbf{x})$.

Given a real vector space $V$, a $\mathbb{Q}$-structure on $V$ is a choice of a $\mathbb{Q}$-linear subspace $V_{0}$ such that $V=V_{0} \otimes_{\mathbb{Q}} \mathbb{R}$ (i.e. there is a basis of $V_{0}$ as a vector space over $\mathbb{Q}$, which is a basis of $V$ as a vector space over $\mathbb{R}$ ). The elements of $V_{0}$ are then called rational points of $V$. If $V_{1}, V_{2}$ are vector spaces with $\mathbb{Q}$-structures, then a linear transformation 
$T: V_{1} \rightarrow V_{2}$ is said to be defined over $\mathbb{Q}$ if it maps rational points to rational points. There is a natural $\mathbb{Q}$-structure on $H^{1}\left(S, \Sigma ; \mathbb{R}^{2}\right)$, namely $H^{1}\left(S, \Sigma ; \mathbb{Q}^{2}\right)$. Since the action of $\operatorname{Mod}(S, \Sigma)$ preserves $H_{1}(S, \Sigma ; \mathbb{Z})$ this induces a well-defined $\mathbb{Q}$-structure on $\mathcal{H}$. Moreover since $\mathcal{H}$ is an affine manifold locally modeled on $H^{1}\left(S, \Sigma ; \mathbb{R}^{2}\right)$, the tangent space to $\mathcal{H}$ at any $x \in \mathcal{H}$ inherits a $\mathbb{Q}$-structure. With respect to this $\mathbb{Q}$-structure, we obtain:

Proposition 6.6. Retaining the notation above, let

$$
\mathcal{O}(x)=\overline{\left\{\operatorname{Rel}_{s}^{(h)} x: s \in \mathbb{R}\right\}} \subset \mathcal{H} \mathcal{T}_{x} .
$$

Then $\mathcal{O}(x)$ is a d-dimensional affine sub-orbifold of $\mathcal{H}$, where $d$ is the dimension of the $\mathbb{Q}$-vector space

$$
\operatorname{span}_{\mathbb{Q}}\left\{\frac{-1}{c_{1}}, \cdots, \frac{-1}{c_{k}}, \frac{1}{c_{k+1}}, \ldots, \frac{1}{c_{\ell}}\right\} \subset \mathbb{R} .
$$

Moreover, for every $x$, the tangent space to $\mathcal{O}(x)$ is a $\mathbb{Q}$-subspace of $H^{1}\left(S, \Sigma ; \mathbb{R}^{2}\right) \cdot{ }^{2}$

Proof. We will work with the standard $m$-torus $\mathbb{T}^{m}=\mathbb{R}^{m} / \mathbb{Z}^{m}$, and define

$$
\Psi: \mathbb{T}^{m} \rightarrow \mathcal{H} \mathcal{T}_{x} ; \quad\left(t_{1}, \ldots, t_{m}\right) \mapsto \Phi\left(c_{1} t_{1}, \ldots, c_{m} t_{m}\right)
$$

Then the conjugacy from Proposition 6.5 leads to a semi-conjugacy from the straight-line flow on $\mathbb{T}^{m}$ in direction

$$
\vec{v}=\left(\frac{-1}{c_{1}}, \cdots, \frac{-1}{c_{k}}, \frac{1}{c_{k+1}}, \cdots, \frac{1}{c_{\ell}}, 0, \ldots, 0\right) .
$$

The orbit closure of the origin of this straight-line flow is a rational subtorus, and the tangent space to the origin is the smallest real subspace $V$ of $\mathbb{R}^{m}$ defined over $\mathbb{Q}$ and containing the vector $\vec{v}$. Every rational relation among the coordinates of $\vec{v}$ gives a linear equation with $\mathbb{Q}$-coefficients satisfied by $\vec{v}$, and vice versa; this implies that the dimension of $V$ is the same as the rational dimension of 6.8.

Similarly to $(6.9)$, let $\widetilde{\Psi}: \mathbb{R}^{m} \rightarrow \mathcal{H}_{\mathrm{m}}$ be defined by $\widetilde{\Psi}\left(t_{1}, \ldots, t_{m}\right)=$ $\widetilde{\Phi}\left(c_{1} t_{1}, \ldots, c_{m} t_{m}\right)$. Then $\widetilde{\Psi}$ intertwines the action of $\mathbb{Z}^{m}$ on $\mathbb{R}^{m}$ by translations, with the action of $\mathrm{M}_{0}(\mathbf{x})$ on the image of $\widetilde{\Psi}$, and we have $\mathcal{O}(x)=\pi(\widetilde{\Psi}(V))$. Let $\vec{v}_{1}, \ldots, \vec{v}_{d}$ be a basis of $V$ contained in $\mathbb{Z}^{m}$, and let $\Gamma \subset \mathbb{Z}^{m}$ be the sub-lattice $\left\langle\vec{v}_{1}, \ldots, \vec{v}_{d}\right\rangle$. The subspace $V$ is fixed by the action $\Gamma \subset \mathbb{Z}^{m}$ by translations, and $\widetilde{\Psi}$ intertwines this translation action with a translation action of a subgroup of $\mathrm{M}_{0}(\mathbf{x})$. Thus in order to prove that the tangent space to $\mathcal{O}(x)$ is defined over $\mathbb{Q}$, it is enough

\footnotetext{
${ }^{2}$ Underlined statements are erroneous. See the Erratum in Appendix B
} 
to prove that any element of $\mathrm{M}_{0}(\mathbf{x})$ acts by translation by a rational vector.

As we have seen (see (6.3)), the action of each $\tau_{i}$ on $H^{1}\left(S, \Sigma ; \mathbb{R}^{2}\right)$ is induced by its action on $H_{1}(S, \Sigma ; \mathbb{Z})$ via

$$
\gamma \mapsto \gamma+\left(\gamma \cap C_{i}\right) C_{i}
$$

i.e. by translating by a vector in $H_{1}(S, \Sigma ; \mathbb{Z})$. Now the assertion follows from the definition of the $\mathbb{Q}$-structure on $H^{1} \overline{\left(S, \Sigma ; \mathbb{R}^{2}\right)} .^{3}$

6.3. Real-rel flow on deformations of the Arnoux-Yoccoz surface. We now specialize further to $x_{r}=\operatorname{Rel}_{r}^{(v)} x_{0}$. Throughout this section we assume that $r>0$ and $r(1-\alpha)$ is not an integral power of $\alpha$. Then $x_{r}$ admits a decomposition into $\mathbf{g}+1$ horizontal cylinders by Theorem $3.7(2)(\mathrm{b})$. Define $\mathcal{O}_{r}=\mathcal{O}\left(x_{r}\right)$ via (6.7), and denote the tangent space to $\mathcal{O}_{r}$ at $x_{r}$ by $T_{r}$. Then we have:

Lemma 6.7. With the notation above, $T_{r}$ is a $\mathbf{g}$-dimensional $\mathbb{Q}$-subspace of $H^{1}\left(S, \Sigma ; \mathbb{R}^{2}\right)$, and $\mathcal{O}_{r}$ is a $\mathbf{g}$-dimensional affine torus.

Proof. By Theorem 3.7(2)(b) the circumferences of the horizontal cylinders on $x_{r}$ are $\alpha^{k+2}, \ldots, \alpha^{k+\mathbf{g}+2}$ for some $k$ depending on $r$. Multiplying through by $\alpha^{k+\mathbf{g}+2}$ in formula (6.8), we see that the dimension of $\mathcal{O}_{r}$ is g. To see that the affine sub-orbifold $\mathcal{O}_{r}$ is a torus (i.e. not its quotient by a nontrivial finite group) we use Proposition 6.2 and the fact that the circumferences of the cylinders are distinct. The other assertions of the Lemma follow from Proposition 6.6.

Define the horizontal twist cohomology subspace $P$ to be the real subspace of $H^{1}\left(S, \Sigma ; \mathbb{R}^{2}\right)$ spanned by the classes of the form $\left(C^{*}, 0\right)$, where $C$ varies over the cylinders of $x_{r}$. As we have seen, $P$ is the tangent space to $\mathcal{H} \mathcal{T}_{r}$, and by Proposition 6.6 the subspace $T_{r}$ lies in $P$ for all $r$ as above. Furthermore we have:

Corollary 6.8. The subspace $P \subset H^{1}\left(S, \Sigma ; \mathbb{R}^{2}\right)$ is $\mathbf{g}+1$ dimensional and is independent of the choice of $r$ selected as above. The action of $\varphi^{*}$ on $H^{1}\left(S, \Sigma ; \mathbb{R}^{2}\right)$ preserves $P$ and has $\left(\operatorname{hol}_{\mathrm{y}}\left(x_{0}\right), 0\right)$ as its dominant eigenvector with eigenvalue $\alpha^{-1}$.

Proof. Recall the holonomy map for the family of surfaces $\left\{x_{r}\right\}$ is

$$
\widetilde{\operatorname{hol}_{y}}: H_{1}(S, \Sigma ; \mathbb{Q}) \rightarrow \mathbb{Q}(\alpha)+\mathbb{Q} r ; \quad \gamma \mapsto \operatorname{hol}_{y}\left(\gamma ; x_{r}\right),
$$

where $r$ is viewed as a free variable. See 3.22 . Because $x_{r}=\operatorname{Rel}_{r}^{(v)} x_{0}$,

$$
\widetilde{\operatorname{hol}}_{y}(\gamma)=\operatorname{hol}_{y}\left(\gamma, x_{0}\right)+\delta(\gamma) r
$$

\footnotetext{
${ }^{3}$ The displacement in period coordinates, $\tau_{i}(\mathbf{x})-\mathbf{x}$, is rational if and only if $\operatorname{hol}\left(C_{i} ; \mathbf{x}\right) \in \mathbb{Q}^{2}$.
} 
where the boundary of $\gamma$ in $H_{0}(\Sigma ; \mathbb{Q})$ is the scalar $\delta(\gamma) \in \mathbb{Q}$ times the class $[\circ]-[\bullet] \in H_{0}(\Sigma ; \mathbb{Z})$.

Lemma 3.10 tells us that $P$ is $\mathbf{g}+1$ dimensional and when restricted to $H_{1}(S, \Sigma ; \mathbb{Q})$ is the set of maps of the form $\left(L \circ \widetilde{h o l}_{y}, 0\right)$ where $L$ : $\mathbb{Q}(\alpha)+\mathbb{Q} r \rightarrow \mathbb{R}$ is $\mathbb{Q}$-linear. Since here we take $r$ as a free variable, $P$ is independent of $r$.

Let $\gamma \in H_{1}(S, \Sigma ; \mathbb{Q})$ be arbitrary. The action of $\varphi^{*}$ on the first coordinate of an element of $P$ is given by

$$
\begin{aligned}
& \left(\varphi^{*}\left(L \circ{\widetilde{\operatorname{hol}_{y}}}_{y}\right)(\gamma)=L \circ{\widetilde{\operatorname{hol}_{y}}}_{y}\left(\varphi^{-1} \gamma\right)\right. \\
& \left.=L\left(\operatorname{hol}_{y}\left(\varphi^{-1} \gamma ; x_{0}\right)+\delta\left(\varphi^{-1} \gamma\right) r\right)\right) \\
& \left.=L\left(\alpha^{-1} \operatorname{hol}_{y}\left(\gamma ; x_{0}\right)+\delta(\gamma) r\right)\right) \text {. }
\end{aligned}
$$

The class $\left(\operatorname{hol}_{y}\left(x_{0}\right), 0\right) \in P$ corresponds to the choice of $L$ which acts trivially on $\mathbb{Q}(\alpha)$ and sends $\mathbb{Q} r$ to zero. From the above, we can see that this class is an eigenvector with eigenvalue $\alpha^{-1}$. Since $\alpha^{-1}$ is Pisot and $\varphi^{*}$ acts as an integer matrix on cohomology, it dominates the action of its $\mathbf{g}-1$ algebraic conjugates. The remaining eigenvector is given by $\gamma \mapsto \delta(\gamma)$ as above (corresponding to an $L$ which annihilates $\mathbb{Q}(\alpha)$ and acts as the identity on $r \mathbb{Q}$ ), which has eigenvalue one.

Theorem 6.9. For any $r$ as above, let $r_{n}=\alpha^{n} r \rightarrow 0$. Then

$$
U x_{0} \subset \overline{\bigcup_{n} \mathcal{O}_{r_{n}}} .
$$

Proof. In the affine orbifold structure on $\mathcal{H}$, the orbit $U x_{0}$ is a line, and the sets $\mathcal{O}_{r_{n}}$ are linear submanifolds. Since $x_{r_{n}} \rightarrow x_{0}$ it suffices to show that the set of accumulation points of the tangent space $T_{r_{n}}$ contains the tangent direction to $U$. By definition of the $U$-action, the derivative of the horocycle flow $\frac{d}{d s}\left[u_{s} x_{0}\right]$ (as an element of the tangent space to $\mathcal{H}$ at $x_{0}$, identified with $\left.H^{1}\left(S, \Sigma ; \mathbb{R}^{2}\right)\right)$ is precisely $\left(\operatorname{hol}_{\mathrm{y}}\left(x_{0}\right), 0\right)$. By Corollary 6.8. $\left(\operatorname{hol}_{\mathrm{y}}\left(x_{0}\right), 0\right)$ is the dominant eigenvector for the action of $\varphi^{*}$ on $P$.

On the other hand taking $\widetilde{g}=D \varphi$ and using Proposition 2.4 we see

$$
\left\{\operatorname{Rel}_{s}^{(h)} x_{r_{n+1}}: s \in \mathbb{R}\right\}=\left\{\operatorname{Rel}_{s}^{(h)} \widetilde{g} x_{r_{n}}: s \in \mathbb{R}\right\}=\left\{\widetilde{g} \operatorname{Rel}_{s}^{(h)} x_{r_{n}}: s \in \mathbb{R}\right\},
$$

and hence $\mathcal{O}_{r_{n+1}}=\tilde{g} \mathcal{O}_{r_{n}}$. Let $P_{1}=\operatorname{span}\left\{\left(\operatorname{hol}_{\mathrm{y}}\left(x_{0}\right), 0\right)\right\} \subset P$. Since $P_{1}$ is generated by an eigenvector, there is a $\varphi^{*}$-invariant complementary subspace $P_{2}$. We claim that the subspace $T_{r} \subset P$ is not contained in $P_{2}$. Assume to the contrary that $T_{r} \subset P_{2}$. Since $P$ has dimension $\mathrm{g}+1$, the subspace $P_{2}$ has dimension $\mathbf{g}$. If $T_{r}$ was contained in $P_{2}$ then by Lemma 6.7 and dimension considerations $T_{r}=P_{2}$. But $P_{2}$ is 
not rational because $\left(\operatorname{hol}_{\mathrm{y}}\left(x_{0}\right), 0\right)$ is not rational. This contradicts the rationality of $T_{r}$; see Lemma 6.7. ${ }^{4}$

Since $T_{r} \notin P_{2}$ there is a vector $v \in T_{r}$ with a nontrivial projection onto $P_{1}$, with respect to the $\varphi$-invariant splitting $P=P_{1} \oplus P_{2}$. Since $\mathcal{O}_{r_{n+1}}=\tilde{g} \mathcal{O}_{r_{n}}$ and $\tilde{g}=D \varphi$, when applying iterates of $\varphi$ to $v$ we get a sequence of vectors in $T_{r_{n}}$ whose span converges projectively to the direction of the dominant eigenvector, as required.

Proof of Theorem 1.4. Let $\mathcal{H}_{0}$ be as in the statement of the Theorem and let $\Omega \subset \mathcal{H}_{0}$ denote the closure of the rel leaf of $x_{0}$. For any $r>0$ and $r$ not an integral power of $\alpha, \Omega$ contains $\mathcal{O}_{r}=\mathcal{O}\left(x_{r}\right)$ (as in (6.7)). Hence by Theorem 6.9, $\Omega$ contains the orbit $U x_{0}$. Applying Corollary 5.8 gives the required conclusion.

\footnotetext{
${ }^{4}$ See the Erratum in Appendix B
} 


\section{Appendix A. Totally Real subextensions}

\section{LIOR BARY-SOROKER, MARK SHUSTERMAN, AND UMBERTO ZANNIER}

For a tower of fields $E \leqslant K \leqslant \bar{E}$ we say that an algebraic extension $M / E$ is totally- $K$ if every embedding of it into $\bar{E}$ over $E$ lands in $K$. Given an algebraic extension $F / E$ we give a bound on its maximal totally- $K$ subextension in case that the absolute Galois group $G_{K}$ is contained in a Sylow subgroup of $G_{E}$ of order prime to $[F: E]$. We use this bound to show that the maximal totally real subfield of $\mathbb{Q}[X] /\left(X^{n}+X^{n-1}+\cdots+X-1\right)$ is $\mathbb{Q}$. This confirms a conjecture made (and empirically tested) by Hooper and Weiss, allowing them to establish the density of the rel leaf of the Arnoux-Yoccoz surface in its connected component in the moduli space of translation surfaces of fixed genus, combinatorial characteristics of singularities, and area.

A.1. Preliminaries. In order to state our main result, we need to fix some notation.

Let $E$ be a field, let $\bar{E}$ be an algebraic closure of $E$, and let $E \leqslant$ $K \leqslant \bar{E}$ be an intermediate subfield. We say that an algebraic extension $M / E$ is totally- $K$ if for every $\tau \in \operatorname{Hom}_{E}(M, \bar{E})$ we have $\operatorname{Im}(\tau) \leqslant K$, or equivalently, if $\operatorname{Hom}_{E}(M, \bar{E})=\operatorname{Hom}_{E}(M, K)$. It is easily verified that the compositum (inside a given field) of a family of totally- $K$ extensions is once again totally- $K$, and that $E / E$ is totally- $K$, so there exists a unique maximal totally- $K$ extension of $E$ inside $\bar{E}$ which we denote by

$$
E_{\mathrm{t}, K}
$$

We also have a relative version of this notion: given an algebraic extension $F / E$, we denote by

$$
E_{\mathrm{t}, K}^{F}:=E_{\mathrm{t}, K} \cap F
$$

the unique maximal totally- $K$ subextension of $F / E$.

For a set $A$, we let $\# A$ be the cardinality of $A$ in case that the latter is finite, and $\infty$ otherwise. We set $[F: E]_{s}:=\# \operatorname{Hom}_{E}(F, \bar{E})$, and use $E \leqslant_{f} F$ to stress that $[F: E]<\infty$. The maximal purely inseparable algebraic extension of $K$ is denoted by $K^{\text {ins }}$, which is just the compositum of all finite extensions $R / K$ with $[R: K]_{s}=1$. A prime number $p$ is said to divide $[F: E]_{s}$ if there exists some

$$
E \leqslant{ }_{f} F_{0} \leqslant F
$$

such that $p \mid\left[F_{0}: E\right]_{s}$. We would like to remind that the automorphism group of an algebraic field extension (e.g. $\operatorname{Aut}(\bar{E} / K))$ carries a topology 
(in our example the open subgroups are $\operatorname{Aut}\left(\bar{E} / K^{\prime}\right)$ for finite extensions $\left.K^{\prime} / K\right)$ with respect to which it is a profinite group.

Recall that a directed poset is a nonempty set $I$ partially ordered by a relation $\leqslant$ such that for every $\alpha, \beta \in I$ there exists some $\omega \in I$ such that $\omega \geqslant \alpha, \beta$. For example, the family of all finite subextensions $E \leqslant f F_{0} \leqslant F$ which we denote by $I_{F / E}$ is a directed poset with respect to inclusion. A subset $J \subseteq I$ is called cofinal if for each $\omega \in I$ there exists some $\pi \in J$ such that $\pi \geqslant \omega$. Note that a cofinal subset is itself a directed poset, and that a subset $J \subseteq I_{F / E}$ is cofinal if and only if $\cup J=F$. Given a function $f: I \rightarrow[0, \infty)$, we say that a point $P \in$ $[0, \infty]$ (viewed as the one-point compactification of the nonnegative real ray $\mathbb{R}_{\geqslant 0}$ ) is a limit point of $f$ if for every neighborhood $V \subseteq[0, \infty]$ of $P$, the inverse image $f^{-1}(V)$ is cofinal. A routine compactness argument shows that $f$ has at least one limit point. We define

$$
\liminf _{I} f
$$

to be the infimum of the set of limit points of $f$, and notice that it is a limit point of $f$.

A.2. Results. Our main result is a bound on the maximal totally- $K$ subextension $E_{\mathrm{t}, K}^{F}$.

Theorem A.1. Let $E$ be a field, let $\bar{E}$ be an algebraic closure, let $E \leqslant K \leqslant \bar{E}$ be an intermediate subfield, and let $F / E$ be an algebraic extension. Suppose that there exists a prime $p$ not dividing $[F: E]_{s}$, such that $\operatorname{Aut}(\bar{E} / K)$ is a pro-p group. Then

$$
\left[E_{\mathrm{t}, K}^{F}: E\right]_{s} \leqslant \liminf _{I_{F / E}}\left|\operatorname{Hom}_{E}\left(-, K^{\mathrm{ins}}\right)\right| .
$$

For simplified (and more concrete) versions of the theorem, where $E$ is assumed to be perfect, see Corollary A.6 and Corollary A.7. From these corollaries we deduce the case of odd $n$ in Corollary A.8. The case of even $n$ follows from the work of Martin (see [M] and the proof of Corollary A.8.

Hooper and Weiss use Corollary A.8 (see Theorem 1.8) as a crucial step in their proof of Theorem 1.4 .

A.3. Preparatory claims. Let $G$ be a group acting on a set $X$, and pick some $x \in X$. We denote by $O_{x}:=\{g x: g \in G\}$ the orbit of $x$, and set $G_{x}:=\{g \in G: g x=x\}$ for the stabilizer of $x$. We call $\left|O_{x}\right|$ the length of the orbit of $x$. Also, $G_{X}$ denotes the (normal) subgroup of all elements of $G$ which act trivially on $X$. 
Proposition A.2. Let $p$ be a prime number, and let $\Gamma$ be a pro-p group acting continuously on a finite set $X$ of cardinality coprime to $p$ ( $X$ carries the discrete topology). Then there is an $x \in X$ such that $\gamma x=x$ for each $\gamma \in \Gamma$.

Proof. Discreteness implies that for each $x \in X$ the subgroup $\Gamma_{x}$ is open in $\Gamma$, so

$$
\Gamma_{X}=\bigcap_{x \in X} \Gamma_{x}
$$

is an open normal subgroup of $\Gamma$ since $X$ is finite. Hence, the action of $\Gamma$ on $X$ factors through the finite $p$-group $P:=\Gamma / \Gamma_{X}$. For each $x \in X$ we have $\left|O_{x}\right|=\left[P: P_{x}\right]$, and $X$ is a finite union of disjoint orbits, so it is impossible for all of the orbits to be of length divisible by $p$ as $p \nmid|X|$. Thus, there is an orbit of length prime to $p$. But, the length of an orbit is the index of a subgroup, so it divides the order of the p-group $P$. We conclude that there exists an orbit of length 1 .

A map $\beta: I \rightarrow J$ between directed posets is called a morphism if for all $x, y \in I$ with $x \geqslant y$ we have $\beta(x) \geqslant \beta(y)$.

Proposition A.3. Let $\beta: I \rightarrow J$ be a surjective morphism of directed posets, let $S \subseteq I$ be a cofinal subset, and let $f: J \rightarrow[0, \infty)$. Then:

(1) The subset $\beta(S) \subseteq J$ is cofinal.

(2) Each limit point of $f \circ \beta$ is a limit point of $f$.

(3) We have the inequality

$$
\liminf _{J} f \leqslant \liminf _{I}(f \circ \beta) .
$$

Proof. In order to establish (1), pick some $j \in J$. Surjectivity provides an $i \in I$ with $\beta(i)=j$. Since $S$ is cofinal, there exists some $s \in S$ such that $s \geqslant i$. We see that $\beta(s) \geqslant \beta(i)=j$ as required. For (2) let $P \in[0, \infty]$ be a limit point of $f \circ \beta$, and let $V$ be a neighborhood of it. This time surjectivity implies that

$$
f^{-1}(V)=\beta\left(\beta^{-1}\left(f^{-1}(V)\right)\right)=\beta\left((f \circ \beta)^{-1}(V)\right)
$$

where the rightmost set is cofinal by (1). Therefore, $f^{-1}(V)$ is cofinal as required. The infimum of a subset (the set of limit points of $f \circ \beta$ ) is never smaller than that of the ambient set (the set of limit points of $f)$ so (3) follows.

Proposition A.4. Let $I$ be a directed poset, and let $\left\{A_{i}\right\}_{i \in I}$ be an inverse system of finite sets. Then

$$
\# \lim _{i \in I} A_{i} \leqslant \liminf _{i \in I}\left|A_{i}\right|
$$


Proof. We may assume that the right hand side takes a finite value $m$, for otherwise there is nothing to prove. Take $V$ to be any open interval of length less than 1 containing $m$, and note that it contains at most one integer. Since $m$ is a limit point of the integer-valued function $i \mapsto\left|A_{i}\right|$, the subset $\left\{i \in I:\left|A_{i}\right| \in V\right\} \subseteq I$ is cofinal, and in particular nonempty, so $V$ contains an integer. We conclude that there exists an integer in every neighborhood of $m$, which means that $m$ is an integer - the unique integer in any such $V$. Therefore,

$$
J:=\left\{i \in I:\left|A_{i}\right|=m\right\} \subseteq I
$$

is cofinal, so it is not difficult (see [RZ, Lemma 1.1.9]) to show that

$$
\# \lim _{i \in I} A_{i}=\# \lim _{j \in J} A_{j} \text {. }
$$

It is therefore sufficient to demonstrate that

$$
X:=\lim _{j \in J} A_{j}
$$

contains at most $m$ distinct elements.

Suppose that there exist distinct $x_{1}, \ldots, x_{m+1} \in X$, and for $j \in J$, denote by $\pi_{j}: X \rightarrow A_{j}$ the projections from the inverse limit. Also, for $s \geqslant t \in J$, we denote by $\tau_{s, t}: A_{s} \rightarrow A_{t}$ the compatible maps of the inverse system. For any $1 \leqslant k \neq \ell \leqslant m+1$ the fact that $x_{k} \neq x_{\ell}$ implies that there exists some $j_{k, \ell} \in J$ such that $\pi_{j_{k, \ell}}\left(x_{k}\right) \neq \pi_{j_{k, \ell}}\left(x_{\ell}\right)$. Since $J$ is directed, there exists some $r \in J$ with $r \geqslant j_{k, \ell}$ for all possible values of $k, \ell$. Let us now show that $\pi_{r}\left(x_{1}\right), \ldots, \pi_{r}\left(x_{m+1}\right)$ are distinct. Pick some $1 \leqslant k \neq \ell \leqslant m+1$ and suppose that $\pi_{r}\left(x_{k}\right)=\pi_{r}\left(x_{\ell}\right)$, so that $\tau_{r, j_{k, \ell}}\left(\pi_{r}\left(x_{k}\right)\right)=\tau_{r, j_{k, \ell}}\left(\pi_{r}\left(x_{\ell}\right)\right)$ which means that $\pi_{j_{k, \ell}}\left(x_{k}\right)=\pi_{j_{k, \ell}}\left(x_{\ell}\right)$ contrary to our choice of $j_{k, \ell}$. We have thus shown that $\left|A_{r}\right|>m$ which is a contradiction to the definition of $J$.

A.4. The proof. For a profinite group $G$, and a subgroup $\Gamma \leqslant G$, we denote by $\langle\Gamma\rangle^{G}$ the minimal closed normal subgroup of $G$ containing $\Gamma$ (i.e. the closure in $G$ of the subgroup generated by the conjugates of $\Gamma$ in $G$ ). We are now ready to establish Theorem A.1. Since we may replace $F$ by any other field isomorphic to it over $E$, we may well assume (by [L, Theorem 2.8]) that $F \leqslant \bar{E}$. Thus, we need to prove:

Theorem A.5. Let $E \leqslant F, K \leqslant \bar{E}$ be field extensions. Suppose that there exists a prime $p$ not dividing $[F: E]_{s}$, such that $\operatorname{Aut}(\bar{E} / K)$ is a pro-p group. Then

$$
\left[E_{\mathrm{t}, K}^{F}: E\right]_{s} \leqslant \liminf _{I_{F / E}}\left|\operatorname{Hom}_{E}\left(-, K^{\mathrm{ins}}\right)\right| .
$$


Proof. Let $E \leqslant F_{0} \leqslant F$ be a finite subextension. Set

$$
\begin{gathered}
G:=\operatorname{Aut}(\bar{E} / E), L:=E_{\mathrm{t}, K}^{F}, L_{0}:=E_{\mathrm{t}, K}^{F_{0}} \\
A:=\operatorname{Hom}_{E}\left(F_{0}, \bar{E}\right), \Gamma:=\operatorname{Aut}(\bar{E} / K), H:=\langle\Gamma\rangle^{G} .
\end{gathered}
$$

First we claim that $H$ fixes $L_{0}$ pointwise. Since $H$ acts continuously on $\bar{E}$ (endowed with the discrete topology) it is sufficient to check that a topological set of generators of $H$ acts trivially on $L_{0}$. Taking $\gamma \in \Gamma, g \in G, \ell_{0} \in L_{0}$ we see that $g \ell_{0} \in K$ so

$$
\left(g^{-1} \gamma g\right) \ell_{0}=g^{-1}\left(\gamma g \ell_{0}\right)=g^{-1}\left(g \ell_{0}\right)=\ell_{0}
$$

since $\gamma$ is trivial on $K$, as claimed.

Since $F_{0} \leqslant \bar{E}$, the inclusion map i: $F_{0} \hookrightarrow \bar{E}$ is in $A$, so $A$ is nonempty, and finite because $|A| \leqslant\left[F_{0}: E\right]<\infty$ (see [L, Theorem 4.1]). Given $g \in G, a \in A$ we see that $g \circ a \in A$, so it is immediate that $G$ acts on $A$ from the left. To see that the action is transitive, pick an $a \in A$ and extend it (using [L, Theorem 2.8]) to some $g \in G$, so that $g \mathfrak{i}=g \circ \mathfrak{i}=$ $\left.g\right|_{F_{0}}=a$ as required for transitivity. In order to show that the action of $G$ on $A$ (viewed as a discrete space) is continuous (i.e. factors through an open normal subgroup), denote by $N$ the normal closure of $F_{0}$ in $\bar{E}$, and note that the action of $\operatorname{Aut}(\bar{E} / N) \triangleleft_{o} G$ on $A$ is trivial since the image of any map $a \in A$ is contained in $N$. Therefore, the action factors through a finite quotient of $G$ as claimed.

Restricting the action to $H$, we get a decomposition of $A$ into disjoint $H$-orbits:

$$
A=\bigcup_{j=1}^{m} H a_{j}
$$

for some $a_{1}, \ldots, a_{m} \in A$, and $m \in \mathbb{N}$. For $1 \leqslant j, k \leqslant m$, take some $g \in G$ for which $g a_{k}=a_{j}$ and recall that $H \triangleleft G$. We thus have

$$
\left|H a_{j}\right|=\left|H g a_{k}\right|=\left|g^{-1} H g a_{k}\right|=\left|H a_{k}\right|
$$

so all the orbits of $H$ share the same length which we denote by $r$. Consequently,

$$
\left[F_{0}: E\right]_{s}=|A|=r m
$$

so $r$ is coprime to $p$, since $|A|$ is coprime to $p$ by our assumption. As $H$ acts trivially on $L_{0}$, we have $H \mathfrak{i} \subseteq \operatorname{Hom}_{L_{0}}\left(F_{0}, \bar{E}\right)$, so

$$
r=|H \mathfrak{i}| \leqslant\left|\operatorname{Hom}_{L_{0}}\left(F_{0}, \bar{E}\right)\right|=\left[F_{0}: L_{0}\right]_{s} .
$$

Since $H$ acts continuously on each of its orbits, so does $\Gamma$. As a result, we have $m$ continuous actions of the pro- $p$ group $\Gamma$ on sets of cardinality prime to $p$. From Proposition A.2, we conclude that there are at least $m$ fixed points for the action of $\Gamma$ on $A$ (at least one fixed point in each orbit of $H$ ). On the other hand, an embedding $\varphi \in A$ 
is a fixed point of $\Gamma$ if and only if its image is contained in the fixed field of $\Gamma$ inside $\bar{E}$ which is $K^{\text {ins }}$ as shown in [L, Proposition 6.11]. Summarizing, we find that

$$
\begin{aligned}
\left|\operatorname{Hom}_{E}\left(F_{0}, K^{\mathrm{ins}}\right)\right| & =\left|A^{\Gamma}\right| \geqslant m \stackrel{\text { A.1 }}{\frac{\left[F_{0}: E\right]_{s}}{r}} \\
& \stackrel{A .2}{\geqslant} \frac{\left[F_{0}: E\right]_{s}}{\left[F_{0}: L_{0}\right]_{s}}=\left[L_{0}: E\right]_{s}
\end{aligned}
$$

where the last equality follows from [L, Theorem 4.1].

Finally, denote by $\beta: I_{F / E} \rightarrow I_{L / E}$ the epimorphism of directed posets given by $\beta\left(F_{0}\right):=L_{0}=E_{\mathrm{t}, K}^{F_{0}}$ and observe that

$$
\begin{aligned}
\liminf _{F_{0} \in I_{F / E}}\left|\operatorname{Hom}_{E}\left(F_{0}, K^{\mathrm{ins}}\right)\right| & \stackrel{A .3}{\geqslant} \liminf _{F_{0} \in I_{F / E}}\left[\beta\left(F_{0}\right): E\right]_{s} \\
& \stackrel{A .3}{\geqslant} \liminf _{L_{0} \in I_{L / E}}\left[L_{0}: E\right]_{s} \\
& =\liminf _{L_{0} \in I_{L / E}}\left|\operatorname{Hom}_{E}\left(L_{0}, \bar{E}\right)\right| \\
& \stackrel{A .4}{\geqslant} \# \lim _{L_{0} \in I_{L / E}} \operatorname{Hom}_{E}\left(L_{0}, \bar{E}\right) \\
& =\# \operatorname{Hom}_{E}\left(\underset{\lim _{0} \in I_{L / E}}{\longrightarrow} L_{0}, \bar{E}\right) \\
& =\# \operatorname{Hom}_{E}(L, \bar{E})=[L: E]_{s}=\left[E_{\mathrm{t}, K}^{F}: E\right]_{s} .
\end{aligned}
$$

A.5. Conclusions. Let us now derive some consequences from our main result. For a field $K$, we denote by $G_{K}$ its absolute Galois group.

Corollary A.6. Let $E \leqslant K \leqslant \bar{E}$ be an extension of the perfect field $E$, and let $F / E$ be a finite extension. Suppose that there exists a prime $p$ not dividing $[F: E]$, such that $G_{K}$ is a pro-p group. Then

$$
\left[E_{\mathrm{t}, K}^{F}: E\right] \leqslant\left|\operatorname{Hom}_{E}(F, K)\right| \text {. }
$$

Proof. This is just a special case of Theorem A.1. Let $K^{\text {sep }}$ be a separable closure of $K$, and note that the separability of $E$ implies that $[M: E]_{s}=[M: E]$ for every finite extension $M / E$. Also, $K$ is perfect since it is an algebraic extension of $E$ (see [L, Corollary 6.12]). Hence,

$$
K^{\text {ins }}=K, \quad G_{K} \cong \operatorname{Gal}\left(K^{\mathrm{sep}} / K\right) \cong \operatorname{Aut}(\bar{E} / K) .
$$

At last, the cofinal subsets of $I_{F / E}$ are those containing $F$, so the lim inf boils down to the value at $F$. 
In view of the primitive element theorem (see [L, Theorem 4.6]), it is natural to "restrict" our attention to field extensions of $E$ of the form $E[X] /(g)$ for some $g(X) \in E[X]$.

Corollary A.7. Let $E \leqslant K \leqslant \bar{E}$ be an extension of the perfect field $E$, and let $g(X) \in E[X]$ be an irreducible polynomial. Suppose that there exists a prime $p$ not dividing $\operatorname{deg}(g(X))$, such that $G_{K}$ is a pro-p group. Then for $F:=E[X] /(g(X))$ we have

$$
\left[E_{\mathrm{t}, K}^{F}: E\right] \leqslant|\{x \in K: g(x)=0\}| \text {. }
$$

Proof. Since $[F: E]=\operatorname{deg}(g(X))$, we may invoke Corollary A.6. An embedding of $F$ into $K$ over $E$ is uniquely determined by its value at $X \in F$ which has to be a root of $g$. Conversely, any root of $g$ in $K$ gives rise to a unique $E$-embedding of $F$ into $K$. Therefore,

$$
\left|\operatorname{Hom}_{E}(F, K)\right|=|\{x \in K: g(x)=0\}|
$$

and the corollary follows.

Corollary A.8. Let $n \geqslant 3$, set $g(X):=X^{n}+X^{n-1}+\cdots+X-1$, and let $L \leqslant \mathbb{Q}[X] /(g(X))$ be a totally real subfield. Then $L=\mathbb{Q}$.

Proof. Set

$$
\begin{aligned}
h(X) & :=-X^{n} g\left(\frac{1}{X}\right)=X^{n}-X^{n-1}-\ldots-X-1, \\
F & :=\mathbb{Q}[X] /(g(X))
\end{aligned}
$$

and note that the splitting field of $g(X)$ over $\mathbb{Q}$ is the same as that of $h(X)$ since the roots of the former are just the inverses of the roots of the latter. By [M, Corollary 2.2], $h(X)$ is irreducible over $\mathbb{Q}$, so $g(X)$ is irreducible over the rationals as well.

Suppose first that $n$ is even. By [M, Theorem 2.6], the Galois group of $h(X)$ over $\mathbb{Q}$ is $S_{n}$, so the same holds for $g(X)$. By the Galois correspondence, $F$ corresponds to a stabilizer of a point in $S_{n}$ which can be naturally identified with $S_{n-1}$, and is readily seen to be a maximal subgroup of $S_{n}$. Since $L$ corresponds to a subgroup of $S_{n}$ containing the aforementioned stabilizer, it follows that either $L=F$ or $L=\mathbb{Q}$. By the arguments preceding [M, Theorem 2.1], $h(X)$ has exactly two real roots, so as usual, $g(X)$ inherits this property. Since $n \geqslant 3, g(X)$ has at least one complex root not in $\mathbb{R}$. We infer that $F$ is not totally real, so necessarily $L=\mathbb{Q}$.

Suppose now that $n$ is odd, and set $E:=\mathbb{Q}, p=2$. Let $\bar{E}$ be the set of all algebraic numbers in $\mathbb{C}$ (this is an algebraic closure of $E$ ) and put $K:=\bar{E} \cap \mathbb{R}$. Observe that $G_{K} \cong \mathbb{Z} / 2 \mathbb{Z}$ which is a pro- $p$ group. By Corollary A.7, $[L: \mathbb{Q}]$ is bounded from above by the number of 
real roots of $g(X)$ which is 1 as shown just before [M, Theorem 2.1]. It follows that $L=\mathbb{Q}$.

As we have already mentioned earlier, Theorem 1.8 reformulates Corollary A.8. In this theorem, the field $F$ is realized as the field obtained by adjoining to $\mathbb{Q}$ the unique root of $g(X)$ in $[0,1]$. Clearly, this field is isomorphic over $\mathbb{Q}$ to $\mathbb{Q}[X] /(g(X))$ (the field in Corollary A.8), so there exists a one to one correspondence between their totally real subfields.

A.6. Acknowledgments. We are grateful to Barak Weiss for telling us about the problem of finding the maximal totally real subfields of number fields arising in dynamics. Special thanks go to Patrick Hooper whose computer verification of Corollary A.8 for all $n \leqslant 1000$ greatly stimulated our work. We would also like to thank Moshe Jarden for his comments on drafts of this work. The first and second authors were partially supported by the Israel Science Foundation grant no. 952/14. The third author was partially supported by the ERC-Advanced grant "Diophantine problems" (grant agreement no. 267273).

\section{Appendix B. ERratum}

Our arguments in $\$ 6$ of this paper contain an error. In this note we explain the error and how to fix it. We are grateful to Florent Ygouf for both pointing out the mistake, and for indicating the correct argument included below. All of the results stated in the introduction of the paper remain valid, as a consequence of the amended argument which will be given below. In the recent preprint [Y], Ygouf proves related results about existence of dense rel leaves in other loci.

B.1. The error. The second assertion of Proposition 6.6 is wrong. Namely, the tangent space to $\mathcal{O}(x)$ is not a $\mathbb{Q}$-space. This affects the validity of Lemma 6.7: while it is true that $T_{r}$ and $\mathcal{O}_{r}$ are gdimensional, it is not true that $T_{r}$ is a $\mathbb{Q}$-subspace. The rationality of $T_{r}$ is used once in the paper, in the proof of Theorem 6.9, at the end of the second paragraph of the proof.

B.2. The area form. Let $\Theta$ be the $\mathbb{R}$-valued anti-symmetric bilinear form on $H^{1}(S, \Sigma ; \mathbb{R})$ defined by $\Theta\left(\beta_{1}, \beta_{2}\right)=\int_{S} \beta_{1} \wedge \beta_{2}$. This bilinear form restricts to the intersection form on $H^{1}(S ; \mathbb{R})$ and can also be used to compute the flat area of a surface, namely the area of the translation surface $x_{0}$ is $\Theta\left(\operatorname{hol}_{x}\left(x_{0}\right), \operatorname{hol}_{y}\left(x_{0}\right)\right)$. See [FM, §3.3] for more information. Since $\Theta$ is defined purely in topological terms, for any homeomorphism $\varphi:(S, \Sigma) \rightarrow(S, \Sigma)$ we have $\Theta\left(\varphi^{*} \beta_{1}, \varphi^{*} \beta_{2}\right)=\Theta\left(\beta_{1}, \beta_{2}\right)$. 
B.3. Correcting the proof of Theorem 6.9. Retain the notation of \$6. We need to justify the claim made in the second paragraph of the proof of Theorem 6.9, that $T_{r} \notin P_{2}$, where $P_{1}=\operatorname{span}\left\{\left(\operatorname{hol}_{y}\left(x_{0}\right), 0\right)\right\}$ and $P_{2}$ is the subspace of $P$ which is $\varphi^{*}$-invariant and complementary to $P_{1}$. Since $\Theta\left(\operatorname{hol}_{x}\left(x_{0}\right), \operatorname{hol}_{y}\left(x_{0}\right)\right)$ is equal to the area of the surface $x_{0}$, it is not 0 , and thus

$$
P_{2}=\operatorname{ker} F \quad \text { for } \quad F: P \rightarrow \mathbb{R}, \quad F(\beta, 0)=\Theta\left(\operatorname{hol}_{x}\left(x_{0}\right), \beta\right) .
$$

We have $\operatorname{dim} P=\mathbf{g}+1, \operatorname{dim} P_{2}=\mathbf{g}$, and by Lemma 6.7, $\operatorname{dim} T_{r}=\mathbf{g}$.

Our proof proceeds by contradiction. Suppose $T_{r} \subset P_{2}$. Then by dimension considerations, $T_{r}=P_{2}$, and in particular, since $T_{r}$ is the tangent space to the torus $\mathcal{O}_{r} \subset \mathcal{H}, \Psi^{-1}\left(\mathcal{O}_{r}\right)$ is a subtorus of $\mathbb{R}^{\mathbf{g}+1} / \mathbb{Z}^{\mathbf{g}+1}$, where $\Psi$ is the map defined in $(6.9)$. Let $\widetilde{\Psi}$ be the map on p. 55. The tangent space to $\Psi^{-1}\left(\mathcal{O}_{r}\right)$ is then $D\left[\widetilde{\Psi}^{-1}\right]\left(T_{r}\right)$. Since this tangent space is parallel to the subtorus $\Psi^{-1}\left(\mathcal{O}_{r}\right)$ of $\mathbb{R}^{\mathbf{g}+1} / \mathbb{Z}^{\mathbf{g}+1}$, any normal vector to $D\left[\widetilde{\Psi}^{-1}\right]\left(T_{r}\right)$ must be proportional to a rational vector.

We claim that a normal vector to $D\left[\widetilde{\Psi}^{-1}\right]\left(T_{r}\right)$ is given by $\left(c_{0}^{2}, c_{1}^{2}, \ldots, c_{\mathbf{g}}^{2}\right)$. To see this, observe that the function $F \circ D \widetilde{\Psi}$ vanishes on $D\left[\widetilde{\Psi}^{-1}\right]\left(T_{r}\right)$. By Proposition 6.1 and (6.9), we have

$$
D \widetilde{\Psi}\left(t_{0}, \ldots, t_{\mathbf{g}}\right)=\left(t_{0} c_{0} C_{0}^{*}+\ldots+t_{\mathbf{g}} c_{\mathbf{g}} C_{\mathbf{g}}^{*}, 0\right) .
$$

Thus,

$$
F \circ D \widetilde{\Psi}\left(t_{0}, \ldots, t_{\mathbf{g}}\right)=\sum_{i=0}^{\mathbf{g}} t_{i} c_{i} \Theta\left(\operatorname{hol}_{x}\left(x_{0}\right), C_{i}^{*}\right) .
$$

For $r>0$, the surface $x_{r}$ is made of $\mathbf{g}+1$ horizontal cylinders as in the discussion in the first paragraph of $\$ 6.1$. The definition of $C_{i}^{*}$ then implies that $C_{i}^{*}\left(\sigma_{j}\right)=\delta_{i j}$ (Kronecker delta) and $C_{i}^{*}$ assigns 0 to all other saddle connections on boundaries of cylinders. The area of $x_{r}$ can be computed by adding the areas of the cylinders, and thus, by the interpretation of $\Theta$ as an area, we see that $\Theta\left(\operatorname{hol}_{x}\left(x_{r}\right), C_{i}^{*}\right)=c_{i}$, the horizontal holonomy of the core curve of $C_{i}$ on $x_{r}$. Since the surfaces $x_{0}$ and $x_{r}$ assign the same horizontal holonomy to absolute periods, we also have $\Theta\left(\operatorname{hol}_{x}\left(x_{0}\right), C_{i}^{*}\right)=c_{i}$, and therefore

$$
F \circ D \widetilde{\Psi}\left(t_{0}, \ldots, t_{\mathbf{g}}\right)=\sum_{i=0}^{\mathbf{g}} t_{i} c_{i}^{2} .
$$

Since we have seen that $T_{r}=\operatorname{ker} F$, this proves the claim.

We have shown that $\left(c_{0}^{2}, c_{1}^{2}, \ldots, c_{\mathbf{g}}^{2}\right)$ is proportional to a rational vector. On the other hand, by Theorem 3.7 we have $\frac{c_{1}}{c_{0}}=\alpha$, and so $\alpha^{2}=$ $\frac{c_{1}^{2}}{c_{0}^{2}} \in \mathbb{Q}$. But, this contradicts that the fact that $\operatorname{dim}_{\mathbb{Q}} \mathbb{Q}(\alpha)=\mathbf{g} \geqslant 3$. 


\section{REFERENCES}

[Arn1] Arnoux, Pierre. Échanges d'intervalles et flots sur les surfaces, Ergodic theory (Sem., Les Plans-sur-Bex, 1980), Monograph. Enseign. Math 29 (1981): $5-38$.

[Arn2] P. Arnoux, Un exemple de semi-conjugaison entre un échange d'intervalles et une translation sur le tore, Bull. Soc. Math. France 116 (1988), 489-500.

[AY] P. Arnoux and J.C. Yoccoz, Construction de difféomorphismes pseudoAnosov, C. R. Acad. Sci. Paris 292 (1981), no. 1, pp. 75-78.

$[\mathrm{AEM}]$ A. Avila, A. Eskin and M. Möller, Symplectic and isometric $\mathrm{SL}_{2}(\mathbb{R})$ invariant subbundles of the Hodge bundle, preprint (2014).

[AHS] A. Avila, P. Hubert and A. Skripchenko, Diffusion for chaotic plane sections of 3-periodic surfaces, Inventiones mathematicae 206 no. 1 (2016): 109-146.

[B] M. Bainbridge, Euler characteristics of Teichmüller curves in genus two, Geom. Topol. 11 (2007), 1887-2073.

[BSW] M. Bainbridge, J. Smillie and B. Weiss, Horocycle dynamics: new invariants and eigenform loci in the stratum $\mathcal{H}(1,1)$, preprint (2016), https://arxiv. org/abs/1603.00808

[Bo] M. D. Boshernitzan, Rank two interval exchange transformations, Ergodic Theory and Dynamical Systems 8 no. 03 (1988), 379-394.

[Bow1] J. Bowman, The complete family of Arnoux-Yoccoz surfaces, Geometriae Dedicata 164 (2013), no. 1, pp. 113-130.

[Bow2] J. Bowman, Orientation-reversing involutions of the genus 3 Arnoux-Yoccoz surface and related surfaces. In: Bonk, M., Gilman, J., Masur, H., Minsky, Y., Wolf, M. (eds.) In the Tradition of Ahlfors-Bers. V, vol. 510 of Contemporary Mathematics, pp. 13-23. American Mathematical Society, Providence, RI (2010).

[CDF] G. Calsamiglia, B. Deroin and S. Francaviglia A transfer principle: from periods to isoperiodic foliations, https://arxiv.org/abs/1511.07635

[DD] R. De Leo and I. A. Dynnikov, Geometry of plane sections of the infinite regular skew polyhedron $\{4,6 \mid 4\}$, Geom. Dedicata 138 (2009), 51-67.

[DH] Vincent Delecroix, W. Patrick Hooper, sage-flatsurf, https://github.com/ videlec/sage-flatsurf. Accessed 12 Aug 2016.

[D1] I. A. Dynnikov, Semiclassical motion of the electron. A proof of the Novikov conjecture in general position and counterexamples, in Solitons, geometry, and topology: on the crossroad, Amer. Math. Soc. Translations (2) 179 (1997) 45-73.

[D2] I. A. Dynnikov, Stability of minimal interval exchange transformations, Conference lecture, Dynamics and Geometry in Teichmüller Space, CIRM, 7-72015.

[DS] I. A. Dynnikov and A. Skripchenko, Symmetric band complexes of thin type and chaotic sections which are not quite chaotic, Transactions of the Moscow Mathematical Society, 76, 251-269.

[EW] M. Einsiedler and T. Ward, Ergodic theory with a view toward number theory, Graduate texts in math. 259 (2011).

[EMi] A. Eskin and M. Mirzakhani, Invariant and stationary measures for the $\mathrm{SL}(2, \mathbb{R})$ action on moduli space. preprint (2014).

[EMiMo] A. Eskin, M. Mirzakhani and A. Mohammadi, Isolation theorems for $\mathrm{SL}_{2}(\mathbb{R})$-invariant submanifolds in moduli space (preprint) 2013. 
[FLP] A. Fathi, F. Laudenbach, and V. Poénaru. "Thurston's work on surfaces, Translated from the 1979 French original by D. M. Kim and D. Margalit." Mathematical Notes, 48, Princeton University Press (2012).

[F] S. Filip, Semisimplicity and rigidity of the Kontsevich-Zorich cocycle, Inventiones mathematicae, 205 (3), 617-670.

[FM] G. Forni and C. Matheus, Introduction to Teichmüller theory and its applications to dynamics of interval exchange transformations, flows on surfaces and billiards, in J. Mod. Dyn. 8 No. 3-4, p. 271-436 (2014).

[H] U. Hamenstädt, Ergodicity of the absolute period foliation, to appear in Israel J. Math.

[HL1] P. Hubert and E. Lanneau, An introduction to Veech surfaces, Handbook of dynamical systems 1, 501-526 (2006).

[HL2] P. Hubert and E. Lanneau, Veech groups with no parabolic element, Duke Math. J. 133(2), 335-346 (2006)

[HW] W. P. Hooper and B. Weiss, The rel leaf and real-rel ray of the Arnoux-Yoccoz surface in genus 3, http://arxiv.org/abs/1506.06773

[HLM] P. Hubert, E. Lanneau, M. Möller, The Arnoux-Yoccoz Teichmüller disc, Geom. Func. Anal. (GAFA) 18 (2009), no. 6, 1988-2016.

[KS] R. Kenyon and J. Smillie, Billiards in rational-angled triangles, Comment. Math. Helv. 75 (2000) 65-108.

[KoZo] M. Kontsevich and A. Zorich, Connected components of the moduli spaces of Abelian differentials with prescribed singularities, Invent. Math. 153 (2003), no. $3,631-678$.

[L] S. Lang, Algebra, Graduate Texts in Mathematics, Springer-Verlag New York 2002.

[LW] S. Lelièvre and B. Weiss, Surfaces with no convex presentations, GAFA 25 (2015) 1902-1936.

[LPV] J. H. Lowenstein, G. Poggiaspalla, and F. Vivaldi, Interval exchange transformations over algebraic number fields: the cubic Arnoux-Yoccoz model, Dyn. Syst. 22 (2007), 73-106.

[M] P. A. Martin, The Galois group of $x^{n}-x^{n-1}-\ldots-1$, Journal of Pure and Applied Algebra, Volume 190, Issues 1-3, 1 June 2004, Pages 213-223.

[Ma] H. Masur, Interval Exchange Transformations and Measured Foliations, The Annals of Mathematics, 2nd Ser., Vol. 115, No. 1. (Jan., 1982), 169-200.

[MaTa] H. Masur and S. Tabachnikov, Rational billiards and flat structures, in Handbook of dynamical systems, Enc. Math. Sci. Ser. (2001).

[McM1] C. T. McMullen, Teichmüller geodesics of infinite complexity, Acta Math., 191 (2003), 191-223.

[McM2] C. T. McMullen, Dynamics of $\mathrm{SL}_{2}(\mathbb{R})$ over moduli space in genus two. Annals of Math. 165 (2007), 397-456.

[McM3] C. T. McMullen, Foliations of Hilbert modular surfaces, Amer. J. Math. 129 (2007), 183-215.

[McM4] C. T. McMullen, Navigating moduli space with complex twists, J. Eur. Math. Soc. (JEMS) 15 (2013) 1223-1243.

[McM5] C. T. McMullen, Moduli spaces of isoperiodic forms on Riemann surfaces, Duke Math. J. 163 (2014) 2271-2323.

[McM6] C. T. McMullen, Cascades in the dynamics of measured foliations, Annales scientifiques de l'ENS. 48 (2015) 1-39. 
[MW] Y. Minsky and B. Weiss, Cohomology classes represented by measured foliations, and Mahler's question for interval exchanges, Annales scientifiques de l'ENS 47 (2014).

[MiWr] M. Mirzakhani and A. Wright, Full rank affine invariant submanifolds, preprint (2016) http://arxiv.org/abs/1608.02147

[Mö] M. Möller, Variations of Hodge structures of a Teichmüller curve, J. Amer. Math. Soc. 19 (2006) 327-344.

[Ra] G. Rauzy, Nombres algébriques et substitutions, Bulletin de la Société Mathématique de France 110 (1982) 147-178.

[RZ] L. Ribes, and P. Zalesskii, Profinite Groups, Ergebnisse der Mathematik und ihrer Grenzgebiete. 3. Folge / A Series of Modern Surveys in Mathematics 40, DOI 10.1007/978-3-642-01642-4, (C) Springer-Verlag Berlin Heidelberg 2010.

[Sch] M. Schmoll, Spaces of elliptic differentials, in Algebraic and topological dynamics, S. Kolyada, Yu. I. Manin and T. Ward eds., Cont. Math. 385 (2005) 303-320.

[SW] J. Smillie and B. Weiss, Minimal sets for flows on moduli space, Isr. J. Math, 142 (2004) 249-260.

[Th] W. Thurston, On the geometry and dynamics of diffeomorphisms of surfaces, Bull. AMS (new series) 19 no. 2 (1988) 417-431.

[V1] W. A. Veech, Gauss measures for transformations on the space of interval exchange maps, Annals of Mathematics (1982) 201-242.

[V2] W. A. Veech, Measures supported on the set of uniquely ergodic directions of an arbitrary holomorohic 1-form, Erg. Th. Dyn. Sys. 19 (1999) 1093-1109.

[W] B. Weiss, Dynamics on parameter spaces: submanifold and fractal subset questions, in Rigidity in Dynamics and Geometry, M. Burger and A. Iozzi, eds, 425-440. Springer (2002).

[Wr1] A. Wright, The field of definition of affine invariant submanifolds of the moduli space of abelian differentials, Geom. Top. (2014).

[Wr2] A. Wright, Translation surfaces and their orbit closures: an introduction for a broad audience, EMS Surv. Math. Sci. 2 no. 1 (2015) 63-108.

$[\mathrm{Y}]$ F. Ygouf, A criterion for density of the isoperiodic leaves in rank 1 affineinvariant orbifolds, preprint (2020) https://arxiv.org/abs/2002.01186

[Zo] A. Zorich, Flat surfaces, in Frontiers in number theory, physics and geometry, P. Cartier, B. Julia, P. Moussa and P. Vanhove (eds), Springer (2006).

City College of New York and CUNy Graduate Center, whooper@ccny.cuny.edu

Tel Aviv University, barakw@post.tau.ac.il

Tel Aviv University, barylior@post.tau.ac.il

Tel Aviv UnIVERSITY, markshus@mail.tau.ac.il

SCuOla Normale Superiore, u.zannier@sns.it 